NBER WORKING PAPER SERIES

\title{
ENDOGENOUS PRODUCTION NETWORKS
}

\author{
Daron Acemoglu \\ Pablo D. Azar \\ Working Paper 24116 \\ http://www.nber.org/papers/w24116
NATIONAL BUREAU OF ECONOMIC RESEARCH
1050 Massachusetts Avenue
Cambridge, MA 02138
December 2017

We thank Nikhil Agarwal, Isaiah Andrews, Ozan Candogan, Chad Jones, Alireza Tahbaz-Salehi, Dan Waldinger and participants in seminars at the Becker-Friedman Institute, Boston University and MIT for suggestions. We gratefully acknowledge financial support from the Bradley Foundation, the Sloan Foundation, the Stanley and Rhoda Fischer Fellowship and the BeckerFriedman Institute. The views expressed herein are those of the authors and do not necessarily reflect the views of the National Bureau of Economic Research.

NBER working papers are circulated for discussion and comment purposes. They have not been peer-reviewed or been subject to the review by the NBER Board of Directors that accompanies official NBER publications.

(C) 2017 by Daron Acemoglu and Pablo D. Azar. All rights reserved. Short sections of text, not to exceed two paragraphs, may be quoted without explicit permission provided that full credit, including (c) notice, is given to the source. 
Endogenous Production Networks

Daron Acemoglu and Pablo D. Azar

NBER Working Paper No. 24116

December 2017

JEL No. C67,E10,E23,L23,O41

\begin{abstract}
$\underline{\text { ABSTRACT }}$
We develop a tractable model of endogenous production networks. Each one of a number of products can be produced by combining labor and an endogenous subset of the other products as inputs. Different combinations of inputs generate (prespecified) levels of productivity. Markets are "contestable" in the sense that production technologies are available to a large number of potential producers. We establish the existence and uniqueness of an equilibrium with an endogenous production network and provide comparative static results on how prices and endogenous technology choices (and thus the production network) respond to changes in parameters. These results show that improvements in technology (or reductions in distortions) spread throughout the economy via input-output linkages and reduce all prices, and under reasonable restrictions on the menu of production technologies, also lead to a denser production network. Using a dynamic version of the model, we show that the endogenous evolution of the production network could be a powerful force towards sustained economic growth. At the root of this result is the fact that the arrival of a few new products expands the set of technological possibilities of all existing industries by a large amount - that is, if there are n products, the arrival of one more new product increases the combinations of inputs that each existing product can use from $2^{n-1}$ to $2^{n}$, thus enabling significantly more pronounced cost reductions from the choice of optimal technology combinations. These cost reductions then spread to other industries that benefit from lower input prices and are further incentivized to adopt additional inputs.
\end{abstract}

\author{
Daron Acemoglu \\ Department of Economics, E52-446 \\ MIT \\ 77 Massachusetts Avenue \\ Cambridge, MA 02139 \\ and CIFAR \\ and also NBER \\ daron@mit.edu \\ Pablo D. Azar \\ MIT Economics Department \\ 50 Ames Street, E19-750 \\ Cambridge, MA, 02139 \\ pazar@mit.edu
}




\section{Introduction}

The production of the same goods typically relies on more complex supply chains in more developed economies. Exxon-Mobil for instance, uses inputs from 65 suppliers from 20 different sectors, including major suppliers in software, computer hardware, and electrical components and equipment. In contrast, the Mexican oil giant PEMEX has 38 suppliers from 13 sectors, and the Venezuelan oil company Petroleos de Venezuela S.A. has 16 suppliers concentrated in nine sectors. Neither has a supplier in software, computer hardware or electrical components and equipment. ${ }^{1}$ These patterns reflect systematic differences at the sectoral level between the three countries. The oil and gas extraction industry in the United States receives inputs from $31.6 \%$ of the other industries in the economy, worth $33 \%$ of its revenues. The same industry in Mexico receives inputs from $20.2 \%$ of industries, worth $7.7 \%$ of its revenues, and in Venezuela, it receives inputs from $27.5 \%$ of industries, worth $11.9 \%$ of its revenues. ${ }^{2}$

The oil and gas industry is not an isolated instance. The input-output matrices of more developed economies are denser than those of developing countries. Once again comparing the United States to Mexico and Venezuela, we see that while the average industry in the United States pays almost $55 \%$ of its revenue stream to intermediate input suppliers, the same numbers are $48.2 \%$ in Mexico and $38.9 \%$ in Venezuela.

What explains the different structures of input usage across these examples? Do these differences contribute to the productivity differences across these economies? In this paper, we take a first step towards answering these questions by developing a tractable framework with endogenous inputoutput linkages. ${ }^{3}$

In our model, each one of $n$ industries decides which subset of the other $n-1$ industries (products) to use as input suppliers, and then how much of each one of these inputs to purchase. Each different input combination leads to a different constant returns to scale production function. What makes our model tractable is that despite the endogeneity of production technology of each industry, the equilibrium is competitive. We achieve this by assuming that markets are "contestable" — meaning that many firms have access to the same menu of technologies. This ensures that in equilibrium, each industry chooses the cost-minimizing quantities of inputs and sets its price equal to this minimal

\footnotetext{
${ }^{1}$ Authors' calculations from Thomson Reuters Value Chains data on Eikon platform. These data are collected from corporate disclosures, corporate filings, and news reports and identify customer-supplier relations.

${ }^{2}$ Authors' calculations. The data used in this and the next paragraph are: for the United States, from the 2007 detailed input-output tables comprising 375 industries; for Mexico, from the 2008 detailed tables comprising 262 industries; and for Venezuela, from the 1997 tables for 120 industries.

${ }^{3}$ For models with exogenous input-output linkages see, among others, Long and Plosser (1983), Ciccone (2002), Gabaix (2011), Jones (2011), Acemoglu, Carvalho, Ozdaglar and Tahbaz-Salehi (2012), Acemoglu, Ozdaglar and Tahbaz-Salehi (2017), Bartleme and Gorodnichenko (2015), Biglio and La'o (2016), Baqaee (2017), Fadinger, Ghiglino and Teteryatnikova (2016), Liu (2017), Baqaee and Farhi (2017), and Caliendo, Parro and Tsyvinski (2017). Some of these papers, such as the last three, allow for non-Cobb-Douglas technologies, and thus allow for the entries of the input-output matrix to be functions of technology and prices. They do not, however, endogenize which sets of input combinations will be used by different sectors.
} 
unit cost, and by the same reasoning, also chooses the cost-minimizing technology. ${ }^{4}$ This typically involves trading off the productivity improvements afforded by different combinations of inputs against the prices of these inputs - in particular, input combinations that yield high productivity relative to the prices of the inputs being utilized are likely to be adopted.

Our first major results establish the existence and generic uniqueness of a competitive equilibrium in this setup. The equilibrium has an intuitive structure, which we exploit to establish several comparative static results. First, when a product adopts additional inputs, this reduces not just its price but all prices in the economy (relative to the wage) - because now this product is a more attractive input to all other industries, enabling them to reduce costs and prices. Second, under a reasonable restriction on the menu of technologies, we establish that a change in technology that makes the adoption of additional inputs more productive for one industry, by reducing prices in the economy, also induces an expansion in the set of input suppliers for all industries. One implication of this economic force, is that industries whose suppliers are more productive face lower input prices, and thus tend to have lower prices and become more likely to be used as inputs themselves. Third, we also show that comparative statics are potentially "discontinuous" - a small change for a single industry can trigger a chain reaction, leading to major shifts in the production structure of many industries. Finally, we demonstrate that distortions discouraging the use of certain inputs by some industries - which may result from taxes or imperfections in contracting institutions will tend to reduce both aggregate productivity and the density of the input-output network. These results are important because we expect the costs and feasibility of setting up and using supplier relationships to vary across countries depending on their contracting institutions and other societal characteristics, paving the way to the formation of different production networks as suggested by our comparison of the oil and gas industry in the United States, Mexico and Venezuela.

We then extend this model to a dynamic setup. While our baseline framework makes essentially no functional form assumptions, for our dynamic analysis we restrict attention to Cobb-Douglas production functions with Hicks-neutral productivity terms (as a function of input combinations). This more tractable special case enables us to investigate the cross-sectional and growth implications of the economic forces identified in our baseline framework.

In our growth analysis, we assume that a new product arrives at each date. We assume that consumers have limited utility benefits from the arrival of additional products, so that economic growth will not result from love-for-variety. Indeed, our first result for the dynamic model establishes that if the input-output structure evolves exogenously, the growth rate of the economy is (almost surely) zero. In contrast to this negative benchmark, we show that when firms select the costminimizing combination of inputs, the economy can achieve sustained growth in the long run. This

\footnotetext{
${ }^{4}$ Throughout, we use the terms "technology choice", "set of inputs" and "input combination" interchangeably. We also use input-output structure (linkages) and production network interchangeably.
} 
growth, like our comparative static results, originates entirely from equilibrium price changes. In particular, growth is fueled by the cost reductions resulting from the selection of the most productive technologies from the new input combinations. Intuitively, with $n$ products in the economy, each industry has a choice between $2^{n-1}$ combinations of inputs. With one more product added to the mix, the number of feasible input combinations increases to $2^{n}$ for each one of the $n$ existing products. The choice of the best technique from this (significantly) expanded set of options leads to nontrivial cost reductions. Crucially, however, economic growth is not just undergirded by the cost reductions enjoyed by the product making the choice, but also by the induced cost reductions that this generates for other industries (as they can now use as input the product that has become cheaper). We show that if the distribution of log productivity of different combinations has sufficiently thick tails (e.g., exponential or Gumbel), this gradually expanded set of options for production techniques engenders exponential growth. ${ }^{5}$

Notably, the origins of growth in our economy are different than those emphasized in the previous literature. First, the nature of growth in our model is connected to but different from the idea of recombinant growth in Weitzman (1999), as well as the related ideas in Auerswald, Kauffman, Lobo and Shell (2000) and Ghiglino (2012). In particular, in contrast to the recombinant growth notion, in our model ideas are not generated by combining, or searching within the set of, all existing ideas; rather, a small trickle of new products significantly expands the input combinations that existing products can use, and this then spreads to the rest of the economy by reducing costs for others. Second, as already hinted at, growth is not driven by the combination of expanded products and love-for-variety (as would be the case in Romer, 1990, or Grossman and Helpman, 1992). Third, it is not a consequence of proportionately more products or innovations arriving over time (as is the case in Romer, 1990, Jones, 1995, Eaton and Kortum, 2001, or Klette and Kortum, 2004). Fourth, it is not driven by proportional improvements in the productivity of all industries as in qualityladder models (as in Aghion and Howitt, 1992, or Grossman and Helpman, 1991). Finally, it is also not due to thick-tailed productivity draws continuously improving technology and spreading in the economy via a diffusion process (as in Akcigit, Celik and Greenwood, 2016, Lucas, 2009, Lucas and Moll, 2014, Perla and Tonetti, 2014). Crucially, even though the distribution of productivity across different input mixes is thick-tailed in our economy, this by itself does not lead to sustained growth. It is the junction of the significant increase in the number of options of input combinations and the endogenous change in the attractiveness of input combinations following changes in prices that underpin growth.

We further use the tractable special case of our model with Cobb-Douglas production technologies and Gumbel-distributed log productivity terms to derive three different sets of empirical

\footnotetext{
${ }^{5}$ If log productivity has an exponential distribution, then the level of productivity has a Pareto distribution, and if log productivity has a Gumbel distribution, then the level of productivity has a Frechet distribution.
} 
predictions from our framework. First, we show that while the distribution of "indegrees" (the number of suppliers per product) has only limited inequality or asymmetry across sectors, the distribution of "outdegrees" (the number of customers of each industry) is much more unequal. This prediction is consistent with the patterns visible in US input-output tables, (e.g., Acemoglu et al., 2012). Moreover, under an additional assumption on the distribution of sectoral shares, we show that the distribution of outdegrees in our model is Pareto - a pattern that also matches the stylized facts documented in Acemoglu et al. (2012). Second, we derive an explicit logistic equation linking the likelihood of an industry being used as a supplier to another industry as a function of its price. Finally, we show that this version of the model can be structurally estimated. We then carry out this structural estimation on US data, and using the estimated parameters, we investigate the implications of marginal distortions and restrictions on sets of input suppliers on the density of the production network and productivity. Our results suggest that both types of distortions impact the equilibrium production network, but the quantitative effects of direct restrictions are much larger than those of marginal distortions and can have sizable effects on aggregate productivity. ${ }^{6}$

In addition to the papers already mentioned, our work is part of a small literature on endogenous input-output linkages. Carvalho and Voigtlander (2015) construct a model in which producers search for new inputs and confront some of the implications of this model with the US input-output tables. Atalay, Hortacsu, Roberts and Syverson (2011) study the choice of suppliers at the firm level. More closely related to our paper are the important prior work by Oberfield (2017) and independent contemporaneous work by Taschereau-Dumouchel (2017). Oberfield constructs an elegant model of the endogenous evolution of the input-output architecture, but with two notable differences from our work. First, at a technical level, Oberfield considers a non-competitive model, which contrasts with our notion of competitive equilibrium. Second and more importantly, but not unrelated to the first, for tractability reasons Oberfield restricts attention to a situation in which each good can only use a single supplier, and obtains an economic mechanism similar to ours - a more productive product is more likely to be used as an input. Nevertheless, Oberfield's framework does not allow an analysis of how the technology choice of an industry affects the structure of input-output linkages for the entire economy, nor does it generate the same type of equilibrium complementarities or sustained growth as our framework. Taschereau-Dumouchel (2017) studies the formation of a production network in the context of business cycle dynamics. Focusing on the social planner's problem, he investigates whether the formation and the response to shocks of equilibrium networks exacerbate economic volatility.

The rest of the paper is organized as follows. The next section introduces our basic model. Equilibrium existence and uniqueness are established in Section 3. Section 4 presents the main

\footnotetext{
${ }^{6}$ This exercise is related to Jones (2011), who investigates the implications of taxes/marginal distortions on aggregate productivity in a world with exogenous input-output linkages. Our focus on the changes in the input-output architecture of the economy and its impact on productivity via this channel is entirely different from Jones's.
} 
comparative static results as well as our extended model with distortions. Section 5 presents our growth model and shows how sustained economic growth can emerge in this setup. Section 6 derives the cross-sectional implications of our model. Section 7 provides structural estimates of the underlying parameters and performs counterfactual exercises to shed light on the role of various distortions on the structure of the input-output network. Section 8 concludes, while the Appendices contain the proofs of the results stated in the text as well as some additional results.

Notation For any pair of $m$-dimensional vectors $\alpha, \beta \in \mathbb{R}^{m}$, we write $\alpha \geq \beta$ if and only if $\alpha_{i} \geq \beta_{i}$ for every $i \in\{1, \ldots, m\}$, and $\alpha>\beta$ if $\alpha \geq \beta$ and there exists at least one $i$ such that $\alpha_{i}>\beta_{i}$. For any two functions $f, g: D \rightarrow \mathbb{R}^{m}$, we write $f \geq g$ if $f(x) \geq g(x)$ for all $x \in D$. If $\alpha \in \mathbb{R}^{n \times m}$ is a matrix, we denote the row vector $\left\{\alpha_{i j}\right\}_{j=1}^{m}$ by $\alpha_{i}$. Unless specified otherwise, we will use lowercase variables to denote logarithms of the corresponding uppercase variables. For example if $P=\left(P_{1}, \ldots, P_{n}\right) \in \mathbb{R}_{>0}^{n}$ is a vector of prices, then $p=\left(p_{1}, \ldots, p_{n}\right) \equiv\left(\log P_{1}, \ldots, \log P_{n}\right)$ will denote the vector of $\log$ prices.

\section{Model}

In this section we introduce our static model and define our notion of equilibrium. We generalize this model to a dynamic setting in Section 5 .

\subsection{Production Technology and Preferences}

There is a set $\mathcal{N}=\{1, \ldots, n\}$ of industries, each producing a single good, denoted by $Y_{i}$ for industry $i$. Throughout, we assume that each industry is contestable in the sense that a large number of firms have access to the same production technology and can enter any sector without any entry barriers. This will ensure that equilibrium profits are always equal to zero. When this will cause no confusion, we work with a representative firm for each industry, and use industry $i$, product $i$ and firm $i$ (for $i \in \mathcal{N}$ ) interchangeably.

Firms in industry $i$ have access to a production technology summarized by

$$
Y_{i}=F_{i}\left(L_{i}, X_{i}, S_{i}, A_{i}\left(S_{i}\right)\right)
$$

Here $L_{i}$ is the amount of labor used by the representative firm in industry $i, S_{i} \subset \varnothing \cup\{1, \ldots, n\} \backslash\{i\}$ denotes the set of (endogenous) suppliers or production technologies used by this industry, $X_{i}=$ $\left\{X_{i j}\right\}_{j \in S_{i}}$ is the vector of intermediate goods used by industry $i$ (where clearly $X_{i j}=0$ if $\left.j \notin S_{i}\right),{ }^{7}$ and $A_{i}\left(S_{i}\right)$ designates the productivity of the technology induced by the use of inputs in the set $S_{i}$ (and for now we do not need to specify its dimension). The dependence of the technology of

\footnotetext{
${ }^{7}$ Clearly, $L_{i}, X_{i}$ and $Y_{i}$ as well as consumption $C_{i}$ are nonnegative for all $i$, but we leave this restriction implicit throughout to simplify the notation.
} 
production on the set of inputs is the crucial feature of our model, and captures the possibility that by combining a richer set of inputs an industry may achieve a greater level of productivity. Motivated by this aspect of input choice, we will interchangeably refer to the choice of $S_{i}$ as technology choice or choice of input suppliers. ${ }^{8}$

We model the consumer side via a representative household whose preferences are given by

$$
u\left(C_{1}, \ldots, C_{n}\right) .
$$

We also assume that the representative household has one unit of labor endowment, which it supplies inelastically. Throughout, we choose the wage as the numeraire, setting

$$
W=1
$$

Denoting the price of good $i$ by $P_{i}$, the budget constraint of the representative household can then be written as

$$
\sum_{i=1}^{n} P_{i} C_{i} \leq 1 .
$$

We next impose some fairly natural assumptions on technology and preferences.

Assumption 1 1. For each $i \in \mathcal{N}, F_{i}\left(L_{i}, X_{i}, S_{i}, A_{i}\left(S_{i}\right)\right)$ is strictly quasi-concave, exhibits constant returns to scale in $\left(L_{i}, X_{i}\right)$, and is strictly increasing and continuous in $A_{i}\left(S_{i}\right), L_{i}$ and $X_{i}$. Moreover, labor is an essential factor of production in the sense that $F_{i}(0, \cdot, \cdot, \cdot) \equiv 0$.

2. $u\left(C_{1}, \ldots, C_{n}\right)$ is continuous, strictly increasing and strictly quasi-concave.

Constant returns to scale on the production side is natural. The strict quasi-concavity of the production function ensures that input demands given technology are uniquely determined, while the feature that output is increasing in the productivity parameters is simply a normalization, enabling us to identify "better technology" with greater $A_{i}\left(S_{i}\right)$. Finally, that labor is essential rules out the extreme possibility that labor can be made redundant by some combination of existing inputs, ensuring that the output level of each industry will always be finite. The assumptions on the utility function of the representative household are fairly minimal. Assumption 1 will be imposed in the next two sections.

\subsection{Firm Optimization}

The contestable market structure combined with constant returns to scale ensures that in each industry, technology and input choices will lead to the lowest unit cost of production. We break

\footnotetext{
${ }^{8}$ In this section, we simplify the analysis by assuming that any combination of inputs is admissible. In Section 4.4 , we show that all of our results generalize to a setting where only a subset of links is (technologically or institutionally) feasible. We further discuss this issue in Appendix C, where we generalize the setup so that some input classes are "essential" for certain sectors (e.g., precision tools need to use at least some metals).
} 
this decision down into two parts: first, the choice of inputs given the set of technologies/suppliers, $S_{i}$, and then the choice of $S_{i}$. The first step can be represented as the following cost minimization problem, where the representative firm in industry $i \in \mathcal{N}$ chooses the vector of intermediate inputs $X_{i}$ and labor $L_{i}$ :

$$
\begin{gathered}
K_{i}\left(S_{i}, A_{i}\left(S_{i}\right), P\right)=\min _{X_{i}, L_{i}} L_{i}+\sum_{j \in S_{i}} P_{j} X_{i j} \\
\text { subject to } F_{i}\left(L_{i}, X_{i}, S_{i}, A_{i}\left(S_{i}\right)\right)=1 .
\end{gathered}
$$

The unit cost function $K_{i}\left(S_{i}, A_{i}\left(S_{i}\right), P\right)$ is explicitly conditioned on the set of inputs, $S_{i}$, because this determines which prices matter for costs, and also captures the dependence of the technology of production and thus the cost function on the set of inputs beyond the productivity shifter $A_{i}\left(S_{i}\right)$. In addition, because $F_{i}$ is strictly increasing and continuous in $A_{i}$, the unit cost function $K_{i}\left(S_{i}, A_{i}, P\right)$ is strictly decreasing and continuous in $A_{i}$.

The second step of firm optimization is the choice of set of technologies to minimize this unit cost function for each $i \in \mathcal{N}$ :

$$
S_{i}^{*} \in \arg \min _{S_{i}} K_{i}\left(S_{i}, A_{i}\left(S_{i}\right), P\right)
$$

\subsection{Equilibrium}

An equilibrium is defined in the usual fashion. In particular, in an economy of size $n$, an equilibrium is represented by $\left(P^{*}, C^{*}, S^{*}, L^{*}, X^{*}, Y^{*}\right)$ such that

1. (Consumer maximization) The vector of consumption $C^{*}$ maximizes (1) subject to (2) given prices $P^{*}$.

2. (Firm maximization) For each $i=1,2, \ldots, n$, factor demands $L_{i}^{*}$ and $X_{i}^{*}$ are a solution to (3), and the technology choice $S_{i}^{*}$ is a solution to (4) given prices $P^{*}$.

3. (Market clearing) For each $i=1,2, \ldots, n$,

$$
C_{i}^{*}+\sum_{j=1}^{n} X_{j i}^{*}=Y_{i}^{*}=F_{i}\left(L_{i}^{*}, X_{i}^{*}, S_{i}^{*}, A_{i}^{*}\left(S_{i}^{*}\right)\right) \text { and } \sum_{j=1}^{n} L_{j}^{*}=1 .
$$

4. (Contestability) For each $i=1,2, \ldots, n$,

$$
K_{i}\left(S_{i}^{*}, A_{i}\left(S_{i}^{*}\right), P^{*}\right)=P_{i}^{*}
$$

The first three conditions are standard. The fourth condition follows from the contestability assumption and imposes that the price for each industry's product has to be equal to the minimum unit cost of production. 
Three observations are useful. First, ours is a version of Samuelson's (1954) "no-substitution economy" where prices are determined entirely on the production side (without reference to consumer preferences) as condition (5) in the definition of equilibrium makes clear. ${ }^{9}$ Second, the labor market clearing condition could have been dropped by Walras's law, but we wrote it as part of market clearing for emphasis. Finally, the vector of equilibrium technology choices $S$ also describes a network - or the equilibrium production network - since it specifies the set of suppliers (technologies used) for each industry.

\subsection{Example: Cobb-Douglas Production Functions with Hicks-Neutral Tech- nology}

The simplest example of production technologies that satisfy part 1 of Assumption 1 is the family of Cobb-Douglas production functions with Hicks-neutral technology, given by

$$
F_{i}\left(L_{i}, X_{i}, S_{i}, A_{i}\left(S_{i}\right)\right)=\frac{1}{\left(1-\sum_{j \in S_{i}} \alpha_{i j}\right)^{1-\sum_{j \in S_{i}} \alpha_{i j}} \prod_{j \in S_{i}} \alpha_{i j}^{\alpha_{i j}}} A_{i}\left(S_{i}\right) L_{i}^{1-\sum_{j \in S_{i}} \alpha_{i j}} \prod_{j \in S_{i}} X_{i j}^{\alpha_{i j}}
$$

for each $i \in \mathcal{N}, A_{i}\left(S_{i}\right)$ is a scalar representing Hicks-neutral productivity, and $S_{i}$ indexes the dependence of the technology on both $A_{i}\left(S_{i}\right)$ and the $\alpha_{i j}$ 's. ${ }^{10}$ We show in the Appendix that the corresponding unit cost function for industry $i$ is

$$
K_{i}\left(S_{i}, A_{i}\left(S_{i}\right), P_{i}\right)=\frac{1}{A_{i}\left(S_{i}\right)} \prod_{j \in S_{i}} P_{j}^{\alpha_{i j}}
$$

This cost function illustrates the tradeoff that the firm faces when it chooses the set $S_{i}$ to minimize costs - anticipated in the Introduction. There might be sets where $\prod_{j \in S_{i}} P_{j}^{\alpha_{i j}}$ is low, but $A_{i}\left(S_{i}\right)$ is high, and sets where $\prod_{j \in S_{i}} P_{j}^{\alpha_{i j}}$ is high, but $A_{i}\left(S_{i}\right)$ is low. The firm will choose a set of suppliers which balances this tradeoff between high productivity and low prices (or vice versa).

Cobb-Douglas production functions enable us to obtain a closed-form solution for equilibrium prices. Let us denote $\operatorname{logs}$ with lower case; that is, $p_{i} \equiv \log P_{i}$ and $a_{i} \equiv \log A_{i}$. We can then write the log unit cost function as a function of log productivities and log prices $k_{i}\left(S_{i}, a_{i}\left(S_{i}\right), p\right)=$ $-a_{i}\left(S_{i}\right)+\sum_{j \in S_{i}} \alpha_{i j} p_{j}$. Since product $i$ 's equilibrium price is equal to its unit cost, equilibrium $\log$ prices will satisfy the system of equations

$$
p_{i}^{*}=\sum_{j \in S_{i}}\left(\alpha_{i j} p_{j}^{*}\right)-a_{i}
$$

\footnotetext{
${ }^{9}$ Samuelson's notion of equilibrium is similar to ours, but imposes an additional condition requiring that the level of consumption of the first good is maximized given the level of consumption of the remaining goods in the economy. Our analysis in the next section shows that Samuelson's additional condition is redundant because the equilibrium price vector is always unique.

${ }^{10}$ The "entropy"-like denominator is included in this production function as a normalization, in particular to simplify the unit cost function derived next. Whether this normalization is present or not makes no difference in our static model. It is also not important in the dynamic model, since it grows at a linear rate as $n \rightarrow \infty$ and thus its presence (or absence) does not affect the asymptotic exponential growth rate of the economy.
} 
Equation (7) admits a closed-form solution for prices. Let $\alpha(S) \in \mathbb{R}^{n \times n}$ be a matrix with

$$
\alpha_{i j}(S)=\left\{\begin{array}{l}
\alpha_{i j} \text { if } j \in S_{i} \\
0 \text { otherwise }
\end{array}\right.
$$

Then given equilibrium technology choices represented by $S^{*}, \log$ prices satisfy

$$
\begin{aligned}
p^{*} & =-\left(I-\alpha\left(S^{*}\right)\right)^{-1} a\left(S^{*}\right) \\
& =-\mathcal{L}\left(S^{*}\right) a\left(S^{*}\right)
\end{aligned}
$$

where $a\left(S^{*}\right)=\left(a_{1}\left(S^{*}\right), \ldots, a_{n}\left(S^{*}\right)\right)^{\prime}$ is the column vector of equilibrium log productivities, and the second line defines the Leontief inverse $\mathcal{L}\left(S^{*}\right) \equiv\left(I-\alpha\left(S^{*}\right)\right)^{-1}$, which will play an important role whenever we work with the Cobb-Douglas production functions. Equation (8) verifies the above claim that prices are determined without reference to consumer preferences in the special case of the Cobb-Douglas family of production functions.

\section{Equilibrium Characterization}

In this section, we first establish the existence of an equilibrium in our static economy and then prove that this equilibrium is generically unique. Existence and uniqueness of equilibrium are not entirely straightforward in this economy, since each industry has a high-dimensional "nonconvex" technology choice. ${ }^{11}$ Nevertheless, we can establish both properties using lattice theoretic tools exploiting the fact that the equilibrium will feature a form of "monotonicity" whereby equilibrium prices of all industries always decline with the adoption of additional (cost-minimizing) technologies by any industry.

\subsection{Existence of Equilibrium}

We start with a lemma that will be useful in proving both existence and uniqueness of equilibrium. The proof of this lemma, like all other proofs in this paper, is presented in Appendix A.

Lemma 1 Suppose Assumption 1 holds. Then given an exogenous network $S_{i}, P^{*}>0$ is an equilibrium price vector if and only if

$$
P_{i}^{*}=K_{i}\left(S_{i}, A_{i}\left(S_{i}\right), P^{*}\right) \text { for each } i=1,2, \ldots, n \text {. }
$$

The "only if" part of this lemma is a direct implication of the definition of a competitive equilibrium, while the "if" part is more substantive and shows that with exogenous networks, any vector of prices equal to unit costs is part of an equilibrium. An important implication of this lemma, which is established as part of its proof, is that given an equilibrium vector of prices, $P^{*}$, there is

\footnotetext{
${ }^{11}$ This nonconvexity is also the reason why we cannot invoke the standard Second Welfare Theorem.
} 
a unique vector of sectoral outputs, $Y^{*}$, consumption levels, $C^{*}$, intermediate input levels, $X^{*}$, and labor demands, $L^{*}$. Using this result, we establish the existence of an equilibrium.

Theorem 1 Suppose Assumption 1 holds. Then an equilibrium $\left(P^{*}, C^{*}, S^{*}, L^{*}, X^{*}, Y^{*}\right)$ exists.

\subsection{Uniqueness of Equilibrium}

In this subsection, we establish the uniqueness of equilibrium prices and generic uniqueness of equilibrium technology choices. In order to establish genericity, we need to specify the exogenous parameters of the economy. Given the uniqueness of equilibrium prices, it is sufficient to focus on a subset of the exogenous parameters corresponding to the shifters of the production technology, $\left\{A_{i}\left(S_{i}\right)\right\}_{i=1}^{n}$. Let us take each $A_{i}\left(S_{i}\right)$ to be represented by an $\ell$-dimensional vector, so that $A_{i}=$ $\left(A_{i}(\varnothing), A_{i}(\{1\}), \ldots, A_{i}(\{1, \ldots, n\} \backslash\{i\})\right)_{i=1}^{n}$ is also a vector in $\mathbb{R}^{\ell \times 2^{n-1}}$, and $A=\left(A_{1}, \ldots, A_{n}\right)$ is a vector

in $\mathbb{R}^{n \times \ell \times 2^{n-1}}$. We define generic uniqueness in terms of the Lebesgue measure on the parameters $A \in \mathbb{R}^{n \times \ell \times 2^{n-1}}$.

Definition 1 The equilibrium network is generically unique if the set

$$
\mathcal{A}=\left\{A: \text { There exist at least two distinct equilibrium networks } S^{*}, S^{* *}\right\}
$$

has Lebesgue measure zero in $\mathbb{R}^{n \times \ell \times 2^{n-1}}$.

Theorem 2 Suppose Assumption 1 holds. Then the equilibrium prices $P^{*}$ and quantities $C^{*}, X^{*}, Y^{*}$ and $L^{*}$ are unique, and the equilibrium network $S^{*}$ is generically unique.

We establish as part of the proof of Theorem 1 that the set of equilibrium prices forms a lattice, which implies that for any two vectors of equilibrium prices, there exists a minimal vector of equilibrium prices. We then show that this is not possible, establishing uniqueness of equilibrium prices and thus quantities. Non-uniqueness of the equilibrium network can only arise if two choices of input combinations give exactly the same unit cost for an industry, which is a non-generic possibility, establishing the generic uniqueness of the equilibrium network.

\section{Comparative Statics}

In this section, we present our main comparative statics results. We first establish that when any industry's technology improves, all industries' prices (weakly) decrease. Then we prove that if the cost function satisfies a (reasonable) single-crossing condition, then an improvement in technology will make the equilibrium network weakly increase. We then show that this single-crossing condition is satisfied when (1) production functions are supermodular; (2) production functions are Cobb-Douglas with Hicks-neutral technologies; or (3) they have a constant elasticity of substitution 
(CES) with input-specific productivity terms. We also show that comparative statics can be "discontinuous" in this model in the sense that small changes in parameters (or distortions) can lead to very large modifications in the production network. Finally, we generalize our model to include distortions and direct restrictions on the formation of links (or adoption of technologies), and derive additional comparative statics.

We should note at the outset that all of our comparative statics work through two complementary channels. The first is a direct channel, whereby, say as a result of an increase in $A_{i}\left(S_{i}\right)$ industry $i$ can reduce its unit cost because it has access to better technology. The second is an indirect channel, where industry $i$ 's technology improvements are transmitted to other industries via prices. If industry $i$ 's price is lower, its customers will face lower unit costs, and then their customers will have lower unit costs as well, and so on. Furthermore, because the choice of network is endogenous, when industry $i$ 's price decreases, other industries are more likely to adopt it as a supplier, decreasing their own costs, which makes the customer industries themselves more likely to be adopted as suppliers.

\subsection{Comparative Statics for Prices}

We first show that any improvement in technologies - in the sense of a shift in the vector of technologies from $A$ to $A^{\prime} \geq A$ - leads to lower prices for all products. Its logic and proof follow those of Theorem 1.

Theorem 3 Suppose Assumption 1 holds. Consider a shift in technology from $A$ to $A^{\prime} \geq A$, and let $P^{*}$ and $P^{* *}$ be the respective equilibrium price vectors. Then $P^{* *} \leq P^{*}$.

Intuitively, an improvement in technology reduces the costs and thus the prices of affected industries. But since the outputs of these industries are used as inputs for the production of other goods in the economy, the prices of all goods tend to decline as a result. Notably, no further assumptions are necessary for this result.

\subsection{Comparative Statics for Technology Choices}

In contrast to prices, the comparative statics for technology choices (equilibrium network) need additional assumptions. This is for two reasons. First, to encourage (or not to discourage) the adoption of an additional product $j$ as an input for industry $i$, we need the "marginal return to adopting $j$ " to increase, but an improvement in the technology for industry $i\left(A^{\prime} \geq A\right.$ as in Theorem 3) does not ensure this. Second, we need to rule out the possibility that the reduction in prices following from the adoption of an additional input by an industry discourages the adoption of additional inputs. The next two definitions introduce the conditions we need to ensure these two features. 
The first one defines a positive technology shock, embedding the notion that the shifting technologies not only improve the level of productivity of different input combinations but also the marginal return from adopting additional input combinations. It also imposes a quasi-submodularity condition, which implies that additional inputs do not directly reduce the productivity from the adoption of yet further inputs. We define these last requirement directly using the unit cost function rather than the production functions - for convenience.

Definition 2 (Positive Technology Shock) A change from $A$ to $A^{\prime}$ is a positive technology shock if

1. (higher level) $A^{\prime} \geq A$;

2. (quasi-submodularity) for each $i \in \mathcal{N}$, and for all $P, K_{i}\left(S_{i}, A_{i}\left(S_{i}\right), P\right)$ is quasi-submodular in $\left(S_{i}, A_{i}\left(S_{i}\right)\right) \cdot{ }^{12}$

The quasi-submodularity condition implies that when $A$ increases to $A^{\prime}$, there are higher marginal returns to adopting a larger set of technologies, as we show in the next lemma.

Lemma 2 Suppose that for each $i \in \mathcal{N}, K_{i}\left(S_{i}, A_{i}\left(S_{i}\right), P\right)$ is quasi-supermodular in $\left(S_{i}, A_{i}\left(S_{i}\right)\right)$. Then for each $i \in \mathcal{N}$, and for all $P$ and for all $S_{i} \subset S_{i}^{\prime}$, we have

$$
K_{i}\left(S_{i}^{\prime}, A_{i}\left(S_{i}^{\prime}\right), P\right)-K_{i}\left(S_{i}, A_{i}\left(S_{i}\right), P\right) \leq 0 \Longrightarrow K_{i}\left(S_{i}^{\prime}, A_{i}^{\prime}\left(S_{i}^{\prime}\right), P\right)-K_{i}\left(S_{i}, A_{i}^{\prime}\left(S_{i}\right), P\right) \leq 0 .
$$

Quasi-submodularity ensures that, holding prices constant, an improvement in technology from $A$ to $A^{\prime}$ encourages the adoption of a larger set of inputs. But as highlighted in Theorem 3, an improvement in technology also leads to lower prices. The next definition introduces the requirement that the return to additional technology adoption does not diminish as prices decline. This is a reasonable, but by no means automatic, restriction (since lower prices mean the cost of buying inputs associated with the new technology is also lower), and we will see that some common production functions satisfy it.

Definition 3 (Technology-Price Single-Crossing Condition) For each $i \in \mathcal{N}$, $K_{i}\left(S_{i}, A_{i}\left(S_{i}\right), P\right)$ satisfies the technology-price single-crossing condition in the sense that for all equilibrium prices $P^{\prime} \leq P$ and $S_{i} \subset S_{i}^{\prime}$, we have

$$
K_{i}\left(S_{i}^{\prime}, A_{i}\left(S_{i}^{\prime}\right), P\right)-K_{i}\left(S_{i}, A_{i}\left(S_{i}\right), P\right) \leq 0 \Longrightarrow K_{i}\left(S_{i}^{\prime}, A_{i}\left(S_{i}^{\prime}\right), P^{\prime}\right)-K_{i}\left(S_{i}, A_{i}\left(S_{i}\right), P^{\prime}\right) \leq 0 .
$$

\footnotetext{
${ }^{12}$ Or more explicitly, for every $S_{i}, T_{i}, A_{i}, P$ we have $K_{i}\left(S_{i}, A_{i}\left(S_{i}\right), P\right) \leq K_{i}\left(S_{i} \cap T_{i}, A_{i}\left(S_{i} \cap T_{i}\right), P\right) \Longrightarrow K_{i}\left(S_{i} \cup\right.$ $\left.T_{i}, A_{i}\left(S_{i} \cup T_{i}\right), P\right) \leq K_{i}\left(T_{i}, A_{i}\left(T_{i}\right), P\right)$ and $K_{i}\left(S_{i}, A_{i}\left(S_{i}\right), P\right)<K_{i}\left(S_{i} \cap T_{i}, A_{i}\left(S_{i} \cap T_{i}\right), P\right) \Longrightarrow K_{i}\left(S_{i} \cup T_{i}, A_{i}\left(S_{i} \cup\right.\right.$ $\left.\left.T_{i}\right), P\right)<K_{i}\left(T_{i}, A_{i}\left(T_{i}\right), P\right)$.
} 
Note that in contrast to the quasi-submodularity condition, this single-crossing condition is a joint restriction on how the unit cost function changes when both the set of inputs and prices change.

The next theorem is our main comparative static result and shows that under the technologyprice single crossing condition, a positive technology shock encourages technology adoption by all industries.

Theorem 4 Suppose Assumption 1 and the technology-price single-crossing condition hold. Then a positive technology shock (weakly) increases the equilibrium network from $S^{*}$ to $S^{* *}$.

Intuitively, a positive technology shock is defined in such a way that it creates direct incentives for technology adoption - for the adoption of additional inputs. This implies that, all else equal, a number of "affected" industries will tend to increase the set of suppliers they use. But this then creates a series of indirect effects, because the use of better technology will reduce their prices. The technology-price single-crossing condition implies that facing lower prices, other industries will also be induced to (weakly) expand their sets of suppliers.

The technology-price single-crossing condition is not always satisfied as we show in Example 1 below. Nevertheless, it is satisfied for a number of important families of production technologies. The next proposition shows that when production functions are supermodular, then this condition is satisfied.

Proposition 1 Suppose $F_{i}\left(L_{i}, X_{i}, A_{i}\left(S_{i}\right), S_{i}\right)$ is supermodular in all its arguments. Then the unit cost function $K_{i}\left(S_{i}, A_{i}\left(S_{i}\right), P\right)$ satisfies the technology-price single-crossing condition.

Even more important in many applications, especially in the presence of input-output linkages, as the family of Cobb-Douglas production functions. The next proposition shows that Cobb-Douglas production functions with Hicks-neutral technology also satisfy the technology-price single crossing condition.

Proposition 2 Suppose $F_{i}\left(L_{i}, X_{i}, A_{i}\left(S_{i}\right), S_{i}\right)$ is in the Hicks-neutral Cobb-Douglas family. Then the unit cost function $K_{i}\left(S_{i}, A_{i}\left(S_{i}\right), P\right)$ satisfies the technology-price single-crossing condition.

The previous two propositions established the technology-price single-crossing condition when the productivity of an industry, and thus its unit cost function, depends on $S_{i}$, the set of inputs it uses. Our next example is more restrictive in this regard in that we consider "input-specific" productivities, meaning that each input has a specific productivity (for the sector in question) which applies regardless of which other inputs are being used. We then show that when production functions are CES with input-specific productivities, the single-crossing property is again satisfied. 
Proposition 3 Suppose $F_{i}\left(L_{i}, X_{i}, A_{i}\left(S_{i}\right), S_{i}\right)$ is a CES function with input-specific productivities, i.e.,

$$
\left(\sum_{j \in S_{i}} \alpha_{i j}\left(A_{i j} X_{i j}\right)^{\frac{\sigma-1}{\sigma}}+\left(1-\sum_{j \in S_{i}} \alpha_{i j}\right) L_{i}^{\frac{\sigma-1}{\sigma}}\right)^{\frac{\sigma}{\sigma-1}}
$$

with $\sigma \neq 1$. Then the unit cost function $K_{i}\left(S_{i}, A_{i}\left(S_{i}\right), P\right)$ satisfies the technology-price singlecrossing condition.

The next example illustrates that the technology-price single-crossing condition is far from vacuous, and might fail even under the quasi-submodularity condition.

\section{Example 1 (Quasi-submodularity does not imply the technology-price single-crossing} condition) Consider an economy with three industries. The production function in each industry is a Cobb-Douglas production function, but crucially technology does not take a Hicks-neutral form, and the input shares of an industry depend on the set of inputs used. Namely,

$$
Y_{i}=\frac{1}{\left(1-\sum_{j \in S_{i}} \alpha_{i j}\left(S_{i}\right)\right)^{1-\sum_{j \in S_{i}} \alpha\left(S_{i}\right)_{i j}} \prod_{j \in S_{i}} \alpha_{i j}\left(S_{i}\right)^{\alpha_{i j}\left(S_{i}\right)}} A_{i}\left(S_{i}\right) L_{i}^{1-\sum_{j \in S_{i}} \alpha_{i j}\left(S_{i}\right)} \prod_{j \in S_{i}} X_{i j}^{\alpha_{i j}\left(S_{i}\right)}
$$

where the conditioning of $\alpha_{i j}$ 's on the set of inputs, $S_{i}$, emphasizes the difference from the family of Cobb-Douglas production functions with Hicks-neutral technology. Suppose also that industries 1 and 2 use only labor as input and have production functions $Y_{1}=e^{-\epsilon} L_{1}$ and $Y_{2}=e^{\epsilon} L_{2}$, where $\epsilon>0$, so that in equilibrium their prices satisfy $p_{1}=-a_{1}=\epsilon$ and $p_{2}=-a_{2}=-\epsilon$, where we have also defined $a_{i}$ (for $i=1,2$ ) as the $\log$ productivities of these two industries. Industry 3, on the other hand, can choose any one of $\varnothing,\{1\},\{2\}$ or $\{1,2\}$ as its set of inputs, with the following input shares

$$
\alpha_{31}(S)=\left\{\begin{array}{l}
0 \text { if } 1 \notin S_{3} \\
\frac{2}{3} \text { if } S_{3}=\{1\} \\
\frac{1}{3} \text { if } S_{3}=\{1,2\}
\end{array} \quad \text { and } \alpha_{32}(S)=\left\{\begin{array}{l}
0 \text { if } 3 \notin S_{3} \\
\frac{2}{3} \text { if } S_{3}=\{2\} \\
\frac{1}{3} \text { if } S_{3}=\{1,2\}
\end{array}\right.\right.
$$

The log productivity for industry 3 is given by $a_{3}(\varnothing)=a_{3}(\{1\})=a_{3}(\{2\})=0$, and $a_{3}(\{1,2\})=\epsilon$. Quasi-submodularity then requires that for all equilibrium prices $\left(p_{1}, p_{2}\right)$,

$$
\begin{aligned}
& \frac{2}{3} p_{2} \leq 0 \Longrightarrow-\epsilon+\frac{1}{3} p_{1}+\frac{1}{3} p_{2} \leq \frac{2}{3} p_{1} \\
& \frac{2}{3} p_{1} \leq 0 \Longrightarrow-\epsilon+\frac{1}{3} p_{1}+\frac{1}{3} p_{2} \leq \frac{2}{3} p_{2}
\end{aligned}
$$

(and also with strict inequalities). It is straightforward to verify that these conditions hold. In particular, because $a_{1}=-\epsilon<0$ and $a_{2}=\epsilon>0$, we have $p_{1}=\epsilon>0$ and $p_{2}=-\epsilon<0$, and thus the first condition is always satisfied as $-\epsilon+\frac{1}{3} p_{2} \leq \frac{1}{3} p_{1}$, while the second condition is also always satisfied because we never have $p_{1} \leq 0$. Hence, the unit cost function for industry 3 is quasi-submodular. 
We next show that it does not satisfy the technology-price single-crossing property. First note that given the equilibrium prices characterized so far, it is cost-minimizing for industry 3 to choose $S_{3}=\{1,2\}$, since its log unit cost with $S_{3}=\varnothing$ is 0 , with $S_{3}=\{1\}$, it is $\frac{2}{3} \epsilon$, with $S_{3}=\{2\}$, it is $-\frac{2}{3} \epsilon$, and with $S_{3}=\{1,2\}$, it takes its lowest value, $-\epsilon$. Next consider a change in the technology of industry 2 so that $a_{2}$ increases to $a_{2}^{\prime}=3 \epsilon$. This can be verified to be a positive technology shock, since we still have $p_{1}>0$ and $p_{2}<0$, and thus the quasi-submodularity condition continues to be satisfied. But following this change, the log unit cost for industry 3 from choosing $S_{3}=\{2\}$ declines to $-2 \epsilon$, while the log unit cost from $S_{3}=\{1,2\}$ declines only to $-\epsilon+\frac{1}{3} \epsilon-\epsilon=-\frac{5}{3} \epsilon>-2 \epsilon$. Therefore, following this positive technology shock industry 3 chooses a smaller set of input suppliers, switching from $\{1,2\}$ to $\{2\}$.

\subsection{Discontinuous Effects}

In this subsection, we show by means of an example that comparative statics are potentially "discontinuous" in that a small change in technologies can lead to a very large reconfiguration of the input-output network. Our example, in particular, shows how following an arbitrarily small increase in one industry's productivity, the equilibrium network can shift from an empty to a complete network. Though, to some degree, this result reflects the discreteness of the choices over the set of suppliers in our model, it is also a consequence of the interdependent nature of technology adoption decisions: the adoption of a productive technology reduces an industry's unit cost of production and makes it more attractive as an input supplier to other industries.

Example 2 (Discontinuous effects) Consider an economy with $n$ industries. Each industry has a Hicks-neutral Cobb-Douglas production function. Given a log price vector p, each industry $i$ chooses a set of suppliers $S_{i}$ which minimizes the log unit cost function $k_{i}\left(S_{i}, a_{i}\left(S_{i}\right), p\right)=-a_{i}\left(S_{i}\right)+$ $\sum_{j \in S_{i}} \alpha_{i j} p_{j}$. The economy's initial $\log$ productivity function is $a_{i}(\varnothing)=0$ and $a_{i}\left(S_{i}\right)=-\epsilon$ for all $i$ and all $S_{i} \neq \varnothing$, where $\epsilon>0$ can be taken to be arbitrarily small. With this configuration of productivities, the unique equilibrium network is empty, i.e., $\left(S_{1}, \ldots, S_{n}\right)=(\varnothing, \ldots, \varnothing)$, and the equilibrium log price vector is $p=0$.

Consider next an exogenous increase in the productivity of industry 1 such that its new log productivity vectored shifts to $a_{1}^{\prime}(\{1, \ldots, n\} \backslash\{1\})=\kappa \epsilon$, and $a_{1}^{\prime}\left(S_{1}\right)=a_{1}\left(S_{1}\right)$ for all $S_{1} \neq\{1, \ldots, n\} \backslash\{1\}$, while there is no change for other industries, i.e., $a_{i}^{\prime}\left(S_{i}\right)=a_{i}\left(S_{i}\right)$ for all $i \neq 1$ and all $S_{i}$. We take

$\kappa>\max _{i} \frac{1}{\alpha_{i 1}}$. The following tattonement process converges to a new equilibrium, represented by network $S^{\prime}$ and $\log$ prices $p^{\prime}$.

In round 0 of the tattonement process, we take the initial price vector $p=0$ as given and allow each industry to minimize costs using their new technology $a^{\prime}$. Therefore, in this round, all industries except industry 1 will choose $S_{i}=\varnothing$, while industry 1 sets $S_{1}=\{1, \ldots, n\} \backslash\{1\}$ and achieves log unit 
(3)

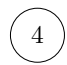

(5)

6
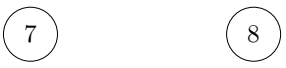

(a) Initial network

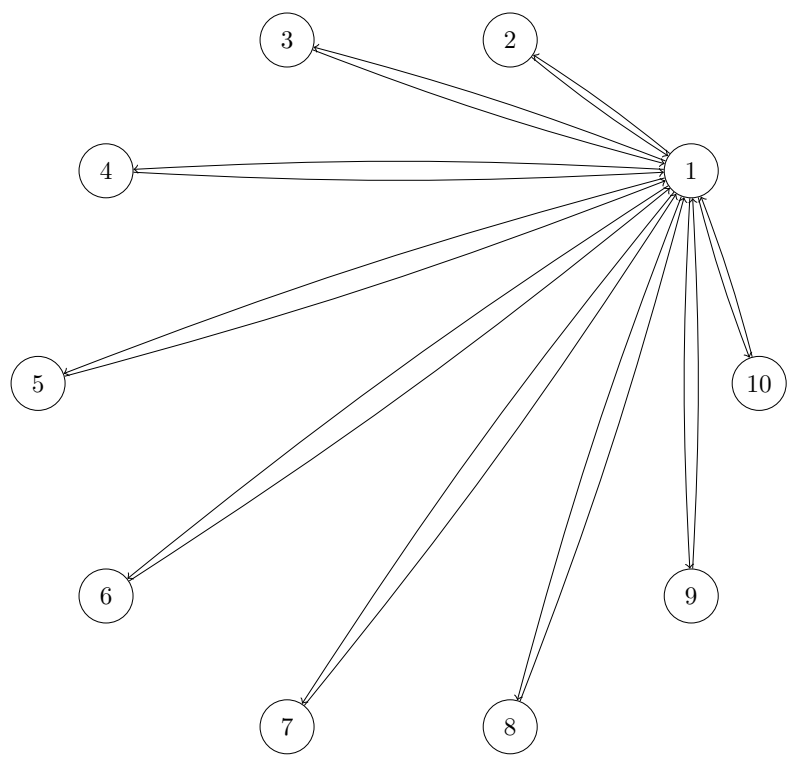

(c) Network after all industries choose industry 1 as a supplier
(1)

(10)

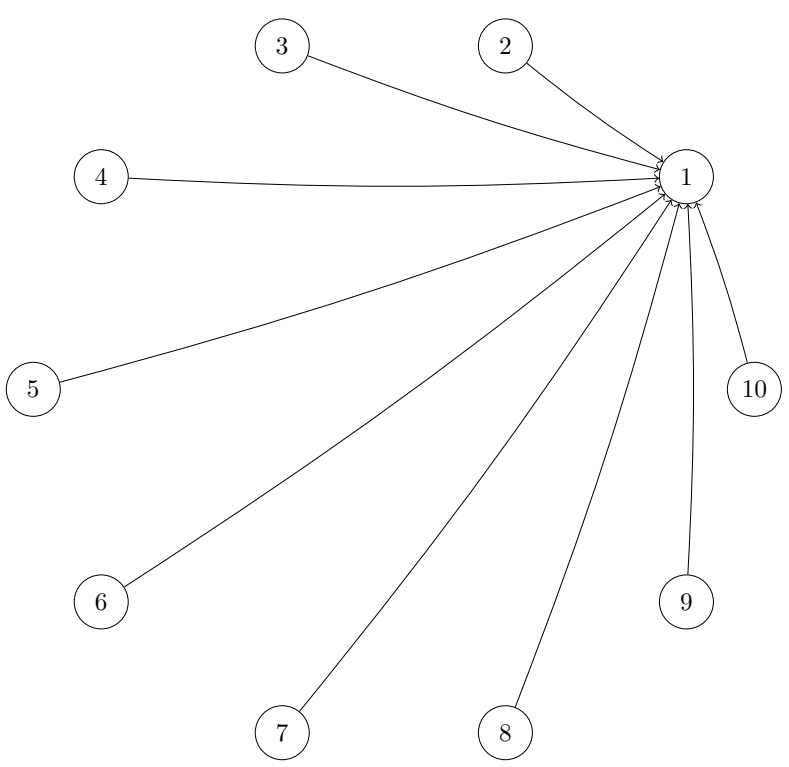

(b) Network immediately after shock to industry 1's productivity

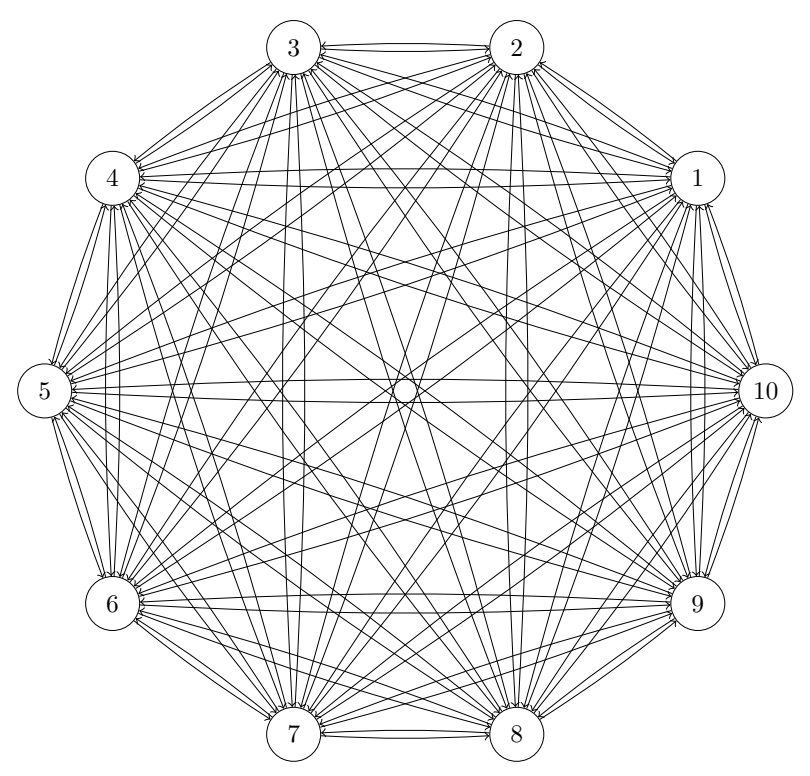

(d) Network after the new equilibrium is reached

Figure 1: Evolution of the input-output network in Example 2 after a shock to industry 1's productivity. Immediately after the shock, industry 1 will adopt all other industries as suppliers. This leads to a drop in industry 1's price. This drop in price is large enough that all other industries adopt industry 1 as a supplier. This leads to a cascade effect of declining prices, until all industries adopt each other as suppliers. 
cost of $\min _{S_{1}} k\left(S_{1}, a_{1}^{\prime}\left(S_{1}\right), p\right)=-a_{1}\left(S_{1}\right)=-\kappa \epsilon$. Consequently, at the end of round 0 , the network is $S^{0}=(\{1, \ldots, n\}-\{1\}, \varnothing, \ldots, \varnothing)$ and the log price vector is $p^{0}=(-\kappa \epsilon, 0, \ldots, 0)$.

In round 1, we now impose the log price vector of $p^{0}$, which resulted from round 0 . Now industry 1 still chooses $S_{1}=\{1, \ldots, n\} \backslash\{1\}$. All other industries will choose industry 1 as a supplier if $\epsilon-\alpha_{i 1} \kappa \epsilon<0$, or equivalently if $\kappa>\frac{1}{\alpha_{i 1}}$. Since we have assumed that $\kappa>\max _{i} \frac{1}{\alpha_{i 1}}$, this condition is satisfied for all industries, and at the end of round 1 , we have $S^{1}=(\{1, \ldots, n\} \backslash\{1\},\{1\}, \ldots,\{1\})$ and a $\log$ price vector of $p^{1}$ such that $p_{i}^{1}<0$ for all $i$.

In round 2 of the tattonement process, we take the log price vector to be $p^{1}$, all of whose elements are negative. This ensures that for each industry $i$, the cost-minimizing set of suppliers is now $S_{i}=\{1, \ldots, n\} \backslash\{i\}$, leading to a vector of log prices of $p^{2} \leq p^{1}<0$.

In round $t \geq 3$, the network of technologies has converged, i.e., $S^{\prime}=S^{3}=S^{2}$, and only prices are updated with

$$
p_{i}^{t}=-a_{i}\left(S_{i}^{\prime}\right)+\sum_{j \in S_{i}^{\prime}} \alpha_{i j} p_{j}^{t-1}
$$

This process converges to $p^{\prime}$. Thus in this example, the small change in productivity shifts the equilibrium technology choices from the empty to the complete network.

\subsection{The Economy with Distortions}

As noted in the Introduction, various taxes and difficulties of writing and enforcing contracts with suppliers make setting up and utilizing supplier relations more expensive in some environments. We next study the implications of two types of distortions on equilibrium prices and input-output networks. The first are sector-specific "marginal distortions," which result from taxes or per-unit contracting costs, and are denoted by $\tau_{j} \geq 0$. These distortions imply that industry $j$ 's goods will be sold (to households and other industries) at the price $P_{j}\left(1+\tau_{j}\right)$. Though, as we will see next, these higher prices will also discourage the use of a broad set of input suppliers, their main impact is on marginal decisions. The second type of distortions we introduce are direct restrictions on the set of suppliers, which might also result from costs of enforcing contracts or from difficulties of setting up complex supply chains. ${ }^{13}$ In particular, we define the set $R=\left\{(i, j) \mid j\right.$ cannot be in $\left.S_{i}\right\}$, which specifies the set of restricted links. Clearly, a set $R^{\prime} \supset R$ represents greater restrictions on input-output linkages than $R$. The definition of equilibrium can be generalized in a straightforward manner to this setting with distortions.

The following theorem generalizes our results so far to this economy with distortions and characterizes the comparative statics with respect to marginal distortions and direct restrictions. Since its proof is very similar to the results presented so far, it is omitted.

\footnotetext{
${ }^{13}$ Though we could also model these costs as fixed costs of setting up links, we opt for the simpler formulation because the characterization of equilibrium becomes less tractable in the presence of such fixed costs.
} 
Theorem 5 Suppose that Assumption 1 holds and let $\tau=\left\{\tau_{j}\right\}_{j=1}^{n}$ denote the vector of marginal distortions and let $R$ denote the set of restricted links. Then an equilibrium with distortions exists and the equilibrium price vector, $P(\tau, R)$, is unique and the equilibrium network, $S(\tau, R)$, is generically unique. Moreover, for any $\tau^{\prime} \geq \tau$ and $R^{\prime} \supset R$, the equilibrium price vectors satisfy $P\left(\tau^{\prime}, R^{\prime}\right) \geq P(\tau, R)$.

As a corollary of Theorem 4, we can show that an increase in either or both types of distortions will lead to a smaller equilibrium network when the technology-price single-crossing condition is satisfied (proof also omitted).

Proposition 4 Suppose that Assumption 1 and the technology-price single-crossing condition hold. Consider a change in distortions from $\tau$ to $\tau^{\prime} \geq \tau$ and from $R$ to $R^{\prime} \supset R$. Then equilibrium networks $S(\tau, R)$ and $S\left(\tau^{\prime}, R^{\prime}\right)$ satisfy $S(\tau, R) \supset S\left(\tau^{\prime}, R^{\prime}\right)$, and

$$
K_{i}\left(S_{i}(\tau, R), A_{i}\left(S_{i}(\tau, R)\right), P(\tau, R)\right) \leq K_{i}\left(S_{i}\left(\tau^{\prime}, R^{\prime}\right), A_{i}\left(S_{i}\left(\tau^{\prime}, R^{\prime}\right)\right), P\left(\tau^{\prime}, R^{\prime}\right)\right)
$$

for all $i \in \mathcal{N}$.

The most substantive implication of Theorem 5 and Proposition 4 is that both marginal distortions, which affect the cost of industry $i$ using industry $j$ 's good as input, and direct restrictions on the set of input-output links lead to a smaller equilibrium input-output network. ${ }^{14}$ Consider first the effects of marginal distortions. These increase (all) prices, and as a consequence, reduce the profitability of using additional inputs. In addition, such distortions increase unit costs and thus reduce productivity. ${ }^{15}$ Turning next to direct restrictions, it is useful to note that $S(\tau, R) \supset S\left(\tau^{\prime}, R^{\prime}\right)$ does not simply reflect the removal of newly-restricted links, but a general reduction in the set of input-output relationships. This is because of indirect effects: once industry $i$ is precluded from using inputs from industry $j$, its unit cost will increase and it will become less likely to be used as an input by other industries.

In Section 7, we investigate the quantitative implications of these two types of distortions for the equilibrium input-output network and GDP. In preparation for that analysis, it is also useful to note that when production functions belong to the Cobb-Douglas family, we can explicitly solve for equilibrium prices in the economy with distortions as $p_{i}^{*}=\sum_{j \in S_{i}}\left(\alpha_{i j}\left(p_{j}^{*}+\log \left(1+\tau_{j}\right)\right)\right)-a_{i}$, and then equation (8) becomes

$$
p^{*}=-\mathcal{L}\left(S^{*}\right) a\left(S^{*}\right)+\mathcal{L}\left(S^{*}\right) \alpha\left(S^{*}\right) \log (1+\tau),
$$

\footnotetext{
${ }^{14}$ We also note that one important example where the technology-price single-crossing condition is automatically satisfied in the presence of marginal distortions is provided by the CES technologies considered in Proposition 3, because marginal distortions are equivalent to input-specific productivities.

${ }^{15}$ The same example presented in the previous subsection can be used to illustrate that small distortions can have significant effects on the equilibrium network and productivity.
} 
where $\tau$ denotes the vector of marginal distortions and $\mathcal{L}\left(\mathcal{S}^{*}\right)$ is the equilibrium Leontief inverse matrix, which depends both on marginal distortions and direct restrictions as characterized in Proposition 4.

\section{Growth with Endogenous Production Networks}

We now extend our baseline model to a dynamic framework, and show how our approach isolates a new economic force with the potential to generate sustained economic growth. For this purpose, we focus on the special case of our baseline model with Hicks-neutral Cobb-Douglas functions. The key economic force towards growth can be understood as follows: If there are $t$ products in the economy, then each industry $i$ has access to $t-1$ possible suppliers, and $2^{t-1}$ ways of combining these suppliers. Selecting the most beneficial combination to achieve a high $A_{i}\left(S_{i}\right)$ and/or low prices for inputs $j \in S_{i}$ will enable the costs of this product to decline and thus its output to increase. ${ }^{16}$

In our dynamic extension of the model, one new industry arrives each period, and all firms have the option of updating their technology by combining the new industry's product with any other subset of products. We ensure that growth is not driven due to expanding product variety by imposing that new products have limited benefits to consumers. But they may have significant benefits in the production process of existing products due to a selection effect - the selection effect allows each industry to choose significantly more productive technologies from the exponentially greater number of options made possible by the arrival of one product.

Our key result in this section shows that this selection effect can generate sustained growth and is in fact essential for growth. If industries are assigned supplier networks exogenously, then the asymptotic growth rate of the economy is (almost surely) zero. In contrast, when industries can select their suppliers, sustained growth is possible. This result also clarifies the new conceptual insight of our model: we can think of the process of economic growth as comprising two modeling blocks: a block determining the arrival of new products or techniques (which we take as exogenous), and a block determining how these products and techniques are combined. While most economic growth models focus on the first block and seek to generate an exponential rate of arrival of new goods and techniques, ours focuses on the second block. In our model, the arrival of new goods is minimal - one good arrives each period. However, even with this minimal arrival rate, we obtain exponential growth from the fact that firms can adopt arbitrary sets of suppliers, and that each combination of intermediate goods generates a different productivity summarized by $A_{i}\left(S_{i}\right)$.

\footnotetext{
${ }^{16}$ As already noted in footnote 8 , Appendix $\mathrm{C}$ generalizes our setup to allow for the possibility that not all input combinations are feasible and some input classes are essential. We show there that this generalization does not affect the analysis of economic growth with endogenous production networks.
} 


\subsection{Model}

There are countably infinite time periods indexed by $t \in\{1,2,3, \ldots\}$. At each time $t$, a new product arrives. Products are indexed by the time at which they arrive, so that the product arriving at time $t$ is referred to as product $t$. Unless it is clear from context, we will index all endogenous variables with time, for example writing $P_{i}(t)$ for the equilibrium price of commodity $i$ at time $t$. Analogously, we will denote the values of $L_{i}, Y_{i}, X_{i j}, C_{i}$, and $S_{i}$ at time $t$ by $L_{i}(t), Y_{i}(t), X_{i j}(t), C_{i}(t)$, and $S_{i}(t)$. The equilibrium wage rate at each $t$ is set as the numeraire, i.e.,

$$
W(t)=1 \text { for all } t
$$

Firms At time $t$, each industry $i \in\{1, \ldots, t\}$ has access to a collection of production technologies indexed by the set of suppliers $S_{i}(t) \subset \varnothing \cup\{1, \ldots, t\} \backslash\{i\}$. The collection of production technologies that firm $i$ has at time $t$ is Cobb-Douglas,

$$
\frac{A_{i}\left(S_{i}(t)\right)}{\left(1-\sum_{j \in S_{i}(t)} \alpha_{i j}\right)^{1-\sum_{j \in S_{i}(t)} \alpha_{i j}} \prod_{j \in S_{i}(t)} \alpha_{i j}^{\alpha_{i j}}} L_{i}(t)^{1-\sum_{j \in S_{i}(t)} \alpha_{i j}} \prod_{j \in S_{i}(t)}\left(X_{i j}(t)\right)^{\alpha_{i j}}
$$

As in our baseline model, adopting (or dropping) new suppliers is costless. This implies that at each point in time, regardless of the way that the households trade off current and future consumption, firms will adopt the cost-minimizing combination of inputs.

Preferences The economy is still inhabited by a representative household, whose time- $t$ preferences we now specialize to the following logarithmic form,

$$
u\left(C_{1}(t), \ldots, C_{t}(t), \beta\right)=\beta_{1} \log \left(C_{1}(t)\right)+\ldots+\beta_{t} \log \left(C_{t}(t)\right)
$$

where the vector $\beta$ satisfies $\sum_{t=1}^{\infty} \beta_{t}=1$ and $\beta_{t} \geq 0$ for all $t$. Crucially, this implies that $\lim _{t \rightarrow \infty} \beta_{t}=$ 0 . This last feature highlights the reason why we have adopted logarithmic preferences - to construct a measure of GDP/utility, and to emphasize that direct utility gains from the addition of new products are minimal. Intuitively, this feature can be justified as follows: we can imagine that the representative household's core necessities are met by goods introduced relatively early in the development process (e.g., hot food, clothing and entertainment), and new goods introduced later (such as microwave ovens, automated textile technologies and streaming) could be useful for more efficiently meeting these necessities, but will not directly increase consumer utility by a large amount. The overall utility of the representative household is given by a discounted sum of its time- $t$ preferences.

In what follows, we will focus on this special case of the economy studied so far, summarized in the next assumption, which strengthens Assumption 1. 
Assumption 1' Production functions are in the Hicks-neutral Cobb-Douglas family and preferences are logarithmic with $\sum_{t=1}^{\infty} \beta_{t}=1$ and $\beta_{t} \geq 0$ for all $t$.

Logarithmic preferences imply that the indirect utility function of the representative household at time $t$ is

$$
V(P(t))=\sum_{i=1}^{t} \beta_{i} \log \frac{\beta_{i}}{P_{i}(t)}=\sum_{i=1}^{t} \beta_{i} \log \beta_{i}-\sum_{i=1}^{t} \beta_{i} \log P_{i}(t) .
$$

This indirect utility function also shows that asymptotically the utility of the representative household, or equivalently log (real) GDP, is inversely proportional to the log price index of the economy, ${ }^{17}$

$$
\pi(t)=\beta(t)^{\prime} p(t) .
$$

Using the fact that the production functions are Cobb-Douglas, equation (8) implies $p(t)=$ $-\mathcal{L}(S(t)) a(S(t))$, where $a(S(t))=\left(a_{1}\left(S_{1}(t)\right), \ldots, a_{n}\left(S_{n}(t)\right)\right)^{\prime}$ is the vector of log productivity terms, and $\mathcal{L}(S(t))$ is the Leontief inverse matrix when the input-output network is given by $S(t)$. Combining the above equations, we get a concise expression for the log price index,

$$
\pi(t)=-\beta(t)^{\prime} \mathcal{L}(t) a(S(t)) .
$$

Since log GDP is proportional to $-\pi(t)=\beta(t)^{\prime} \mathcal{L}(S(t)) a(S(t))$, we define the asymptotic GDP growth rate as: ${ }^{18}$

$$
g^{*}=\lim _{t \rightarrow \infty}\left(-\frac{\pi(t)}{t}\right) .
$$

These expressions also emphasize that equilibrium GDP and growth depend both on the direct effects of productivity as captured by the $a(S(t))$ matrix and the indirect effects working through the equilibrium Leontief inverse, $\mathcal{L}(S(t))$.

We can also anticipate that similar expressions apply in the presence of distortions. In particular, in this case the log price index is given as

$$
\begin{aligned}
\pi(t) & =\beta(t)^{\prime}(p(t)+\log (1+\tau)) \\
& =-\beta(t)^{\prime} \mathcal{L}(t)(a(S(t)))+\beta(t)^{\prime} \mathcal{L}(t) \alpha(t) \log (1+\tau)+\beta^{\prime} \log (1+\tau) \\
& =-\beta(t)^{\prime} \mathcal{L}(t)(a(S(t))-\log (1+\tau)),
\end{aligned}
$$

where the last equality is a consequence of the fact that $\mathcal{L}(t)=\mathcal{L}(t) \alpha(t)+I$. This Leontief inverse depends both on marginal distortions, $\tau$, and on direct restrictions on input-output linkages introduced in Section 4.4.

\footnotetext{
${ }^{17}$ Because household income is normalized to 1 by our choice of numeraire, log real GDP is equal to the indirect utility function. This utility function contains two terms. The first one, $\sum_{i=1}^{t} \beta_{i} \log \beta_{i}$, asymptotes to constant as $t \rightarrow \infty$ in view of the fact that $\lim _{t \rightarrow \infty} \beta_{t}=0$, and thus asymptotically, log GDP is given by a constant plus the negative of this log price index.

${ }^{18}$ An alternative, perhaps more conventional, definition would have been $\lim _{t \rightarrow \infty}(-\Delta \pi(t))$. When this limit exists, then it is straightforward to see that $g^{*}=\lim _{t \rightarrow \infty}(-\Delta \pi(t))$. However, this limit may fail to exist, even though $g^{*}$ is well defined (e.g., because $-\Delta \pi(t)$ fluctuates between high and low values even asymptotically). Our definition thus avoids these inessential complications.
} 


\subsection{Lack of Growth With Exogenous Networks}

We first establish that when the set of suppliers for each product is taken as "exogenous," sustained growth is not possible, even though new goods are arriving at each period as specified above. By the set of suppliers being exogenous we mean that each industry is given the option of using a specific new technology (set of suppliers) at each date, and decides between its current technology and this new option. We will show that, under two relatively mild assumptions stated next, the growth rate of this economy with exogenous networks will be almost surely equal to zero.

Assumption 2 The log productivity terms $a_{i}\left(S_{i}\right)$ are drawn i.i.d. from a distribution $\Phi$. There exists a constant $D>0$ such that, if $Z_{1}, \ldots, Z_{n}$ are drawn i.i.d. from $\Phi$, then $\lim _{n \rightarrow \infty} \frac{\max _{i \in\{1, \ldots, n\}} Z_{i}}{\log _{2} n}=$ $D$ almost surely.

Assumption 3 1. There exists $\theta<1$ such that $\sum_{j=1}^{\infty} \alpha_{i j} \leq \theta$ for all $i \in \mathbb{N}$.

2. Furthermore, for every $\epsilon>0$, there exists a constant $T$ such that for all $i \in \mathbb{N}, \sum_{j=T}^{\infty} \alpha_{i j} \leq \epsilon$.

Assumption 2 rules out log productivity distributions that have either too thin or too thick tails. We show in Appendix B that the Gumbel and exponential distributions satisfy Assumption 2. In contrast, finite and normal distributions do not satisfy this assumption because their tails decrease at a faster than exponential rate. When log productivity follows a Pareto or Frechet distribution, this assumption is not satisfied either, this time because their tails decrease at a slower rate than exponential.

The first part of Assumption 3 imposes that the matrix norm $\|\alpha(S(t))\|_{\infty}=\max _{i} \sum_{j}\left|\alpha_{i j}(S(t))\right|$ is uniformly bounded for all $t$, and implies that $\|\mathcal{L}(S(t))\|_{\infty} \leq \sum_{\ell=0}^{\infty}\left\|\alpha^{\ell}(S(t))\right\|_{\infty} \leq \frac{1}{1-\theta}$. This bound is a dynamic analogue of our requirement in Assumption 1 that labor is an essential factor of production. Without this assumption, the share of labor in each industry could asymptote to zero. ${ }^{19}$ The second part states that goods invented relatively early on are not just more important for consumption (as imposed in Assumption 1'), but they also make up the more important inputs in the sense that the sum of the shares of inputs arriving after some time $T$ are uniformly bounded. This property will be used only in the proof of Theorem 7, and will enable us to show that the upper and lower bounds we establish for the rate of growth of the economy with endogenous production networks are the same.

The next theorem shows that under Assumption 2 and without the endogenous choice of production network, the expansion of the set of goods in this economy cannot generate sustained growth.

\footnotetext{
${ }^{19}$ In fact, Theorem 6 can be established when both of these assumptions are relaxed further. However, since these assumptions are useful for our next result, Theorem 7, we simplify the exposition by imposing them at this point.
} 
Theorem 6 Suppose that Assumptions 1', 2 and 3 hold. At each time $t \geq 1$, a set of suppliers $S_{i}^{O}(t) \subset\{1, \ldots, t\} \backslash\{i\}$ for each $i \in \mathcal{N}$ is selected uniformly at random. Then each industry $i$ chooses between its existing set of suppliers, $S_{i}^{*}(t-1)$, and $S_{i}^{O}(t)$. Then $g^{*}=0$ almost surely.

Intuitively, there are only limited utility gains from the introduction of new consumption goods (because $\lim _{t \rightarrow \infty} \beta_{t}=0$ as noted above). Thus sustained growth can result only if new products significantly improve the productivity of existing goods. But under Assumption 2, this cannot happen when new goods are introduced into the supply chain in a random manner. This result, however, does not preclude the possibility of sustained growth when firms select which inputs to introduce into their production process as we see next.

\subsection{Growth With Endogenous Networks}

The main result of this section is that, in contrast to the negative result of the previous subsection, sustained economic growth will result when firms can select their set of suppliers. Our negative result showed that growth with exogenous networks occurred only on a set of measure zero. We now prove that the economy with endogenous networks almost surely has a positive long-run growth rate.

Theorem 7 Suppose that Assumptions 1', 2 and 3 hold, and let $D>0$ be as defined in Assumption 2. Each industry chooses its set of suppliers $S_{i}^{*}(t) \subset\{1, \ldots, t\} \backslash\{i\}$. Then for each $i \in \mathcal{N}$, the equilibrium log price vector $p^{*}(t)$ satisfies,

$$
\lim _{t \rightarrow \infty}-\frac{p_{i}^{*}(t)}{t \sum_{j=1}^{t} \mathcal{L}_{i j}}=D>0 \text { almost surely, }
$$

and thus

$$
g^{*}=D \sum_{i, j=1}^{\infty} \beta_{i} \mathcal{L}_{i j}>0 \text { almost surely }
$$

Therefore, in stark contrast to Theorem 6, when firms can choose their input suppliers in an unrestricted fashion, the economy (almost surely) achieves sustained growth. The selection effect - the fact that out of the many new input combination options presented to them, each industry chooses the cost-minimizing combination of inputs - is at the root of this sustained growth result. ${ }^{20}$

This result also illustrates the direct and indirect effects that the arrival of new technologies has on prices. The direct effect is that as each industry $i$ faces an expanded set of possible input combinations, its costs and thus equilibrium price decline. The indirect effect comes from the fact that, as each industry $i$ 's prices decline, industries that use this industry's output as input will also

\footnotetext{
${ }^{20}$ Of course, if the exogenous draws of the input-output network sequence in Theorem 6 happened to coincide with the endogenously chosen sequence of the input-output network in Theorem 7 , the exogenous network economy would also generate sustained growth, but clearly this event has probability zero.
} 
benefit because their costs will decrease. In particular, recall that $-p_{i}^{*}(t)=\sum_{j=1}^{t} \mathcal{L}_{i j}(S) a_{j}\left(S_{j}\right)$. We can measure the direct effect by counterfactually setting the prices of all intermediate inputs for industry $i$ to $P_{j}(t)=1$ (for $j \neq i$ ) so that the cost reductions of industries that adopt new technologies do not benefit their customers. In this case, we would have that the log price of all intermediate inputs is zero, and the unit cost of producing good $i$ becomes $-p_{i}^{*}(t)=a_{i}\left(S_{i}\right)$. Industry $i$ would then choose $S_{i}$ to maximize $a_{i}\left(S_{i}\right)$ and consumers would face the price $p_{i}=-\max _{S_{i}} a_{i}\left(S_{i}\right)$. The log GDP level would then be $\sum_{i=1}^{t} \beta_{i} \max _{S_{i}^{\prime}} a_{i}\left(S_{i}^{\prime}\right)$, just capturing the direct effect. The indirect effect is the difference between this quantity and the actual log GDP level, $\frac{1}{t} \sum_{i, j=1}^{t} \beta_{i} \mathcal{L}_{i j}(S) a_{j}\left(S_{j}\right)$, which includes the indirect effects working through the Leontief inverse matrix $\mathcal{L}(S)$.

The next three corollaries clarify the economic forces that generate sustained growth in our model. Because their proofs are minor variations on the proof of Theorem 7 , the proofs of Corollaries 1 and 2 are straightforward and will be omitted.

The first corollary shows that it is sufficient for a subset of industries to be able to choose their suppliers in an unconstrained manner.

Corollary 1 Suppose that there exists a finite, nonempty set $\mathcal{S}$ of industries for which Assumptions $1^{\prime}, 2$ and 3 hold and that can choose their sets of suppliers $S_{i}^{*}(t) \subset \varnothing \cup\{1, \ldots, t\} \backslash\{i\}$. The remaining industries can not choose their suppliers. Then

$$
g^{*}=D \sum_{i, j=1}^{\infty} \beta_{i} \mathcal{L}_{i j}>0 \text { almost surely }
$$

This corollary thus shows that sustained (exponential) growth does not require the conditions of Theorem 7 for all industries. Though the growth rate in this corollary has exactly the same form of that in Theorem 7 , it will be generally quite different (smaller than) that rate, because the equilibrium Leontief inverse, $\mathcal{L}$, will be different (in fact, element-wise smaller).

A restrictive feature of Theorem 7 is that Assumption 2 requires $\max _{i \in\{1, \ldots, n\}} X_{i}$ to scale exactly with $\log _{2} n$. The next corollary shows that if this is not exactly the case, sustained growth still emerges provided that there is more than one new product arriving at each date.

Corollary 2 Suppose that Assumptions 1', and 3 hold and that each industry chooses its set of suppliers $S_{i}^{*}(t) \subset\{1, \ldots, t\} \backslash\{i\}$. In addition, instead of Assumption 2, we have that for any $X_{1}, \ldots, X_{n}$ that are drawn i.i.d. from $\Phi, \lim _{n \rightarrow \infty} \frac{\max _{i \in\{1, \ldots, n\}} X_{i}}{\left(\log _{2} n\right)^{\eta}}=D$ almost surely, where $\eta<1$. Suppose also that at date $t$, there are $\frac{t^{\frac{1-\eta}{\eta}}}{\eta}$ new products (so that the total number of products at time $t$ is $t^{\frac{1}{\eta}}$ ). Then

$$
g^{*}=D \sum_{i, j=1}^{\infty} \beta_{i} \mathcal{L}_{i j}>0 \text { almost surely }
$$

Finally, the next corollary derives the asymptotic growth rate for an economy with distortions. We will use this result in our counterfactual analysis in Section 7. 
Corollary 3 Suppose that Assumptions 1', 2 and 3 hold, each industry chooses its set of suppliers $S_{i}^{*}(t) \subset\{1, \ldots, t\} \backslash\{i\}$, there are marginal distortions represented by the vector $\tau$, and each link $(i, j)$ is assigned to the set of restricted links $R$ independently at random with probability $\rho$. Then

$$
g^{*}=(1-\rho) D \sum_{i, j=1}^{\infty} \beta_{i} \mathcal{L}_{i j}>0 \text { almost surely }
$$

We reiterate that, though the growth rate in all three corollaries has an identical or at the very least, a very similar expression to that in Theorem 7 , the Leontief inverse matrix, $\mathcal{L}$, will be in general different, leading to a different equilibrium growth rate.

\section{Cross-Sectional Implications}

In this section, we draw the cross-sectional implications of our model of endogenous production network. Our focus will be on a static economy with large $n$, which will enable us to draw on some of the results developed in the context of our study of economic growth in the previous section. ${ }^{21}$ Throughout this section we impose Assumption 1', ensuring that all production functions are Cobb-Douglas and preferences are logarithmic, and also strengthen Assumption 2 so that log productivity terms are distributed according to a Gumbel distribution. Under these assumptions, we first establish a closed-form characterization of the probability of industry $j$ to be adopted as a supplier to industry $i$. We then prove the main result of this section, showing that under a stronger version of Assumption 3 on the shape of the $\alpha_{i j}$ parameters, the distribution of indegrees is concentrated (thus exhibiting limited inequality), while the distribution of outdegrees is much more unequal. In other words, industries are similar in terms of how many inputs they use, but they are very different in terms of how many other industries they supply. This contrast is in line with the patterns visible from the US input-output tables (e.g., Acemoglu et al., 2012). Finally, under an additional assumption on $\alpha_{i j}$ 's, we show that the distribution of outdegrees has an approximate power law distribution (a Pareto tail), which implies a high degree of inequality in the propensity of some industries to be used as suppliers to others, again similar to what Acemoglu et al. (2012) document for the US economy.

\subsection{Closed-form Expressions for Edge Probabilities}

In the rest of this section, we work under the following strengthening of Assumption 2.

Assumption $2^{\prime}$ The $\log$ productivity terms $a_{i}\left(S_{i}\right)=\log A_{i}\left(S_{i}\right)$ are drawn i.i.d. from a Gumbel distribution with $\operatorname{cdf} \Phi(x ; \sigma)=e^{-e^{-x / \sigma}}$.

\footnotetext{
${ }^{21}$ Our cross-sectional results can also be developed in the context of a growing economy as in the previous section. We focus on the static economy for simplicity.
} 
This assumption enables us to compute a closed-form, logistic expression for the probability that an edge $(i, j)$ is present in the production network.

Lemma 3 Suppose Assumptions $1^{\prime}$ and $2^{\prime}$ hold. Then:

1. Conditional on the price vector $P$, the probability of industry $j$ choosing $S_{i}$ as its set of suppliers is

$$
\operatorname{Pr}\left(S_{i} \mid P\right)=\frac{e^{-\sum_{j \in S_{i}} \frac{\alpha_{i j}}{\sigma} p_{j}}}{\sum_{S_{i}^{\prime}} e^{-\sum_{j \in S_{i}^{\prime}} \frac{\alpha_{i j}}{\sigma} p_{j}}}=\frac{\prod_{j \in S_{i}} P_{j}^{-\frac{\alpha_{i j}}{\sigma}}}{Z_{i}}
$$

2. Conditional on the price vector $P$, the probability that industry $j$ is a supplier to industry $i$ is

$$
\operatorname{Pr}\left(j \in S_{i} \mid P\right)=\frac{P_{j}^{-\frac{\alpha_{i j}}{\sigma}}}{1+P_{j}^{-\frac{\alpha_{i j}}{\sigma}}} .
$$

Lemma 3 also yields a simple logistic equation for the expected outdegree - or number of customers — of industry $j$ :

$$
\sum_{i \in \mathcal{N}} \operatorname{Pr}\left(j \in S_{i} \mid P\right)=\sum_{i \in \mathcal{N}} \frac{P_{j}^{-\frac{\alpha_{i j}}{\sigma}}}{1+P_{j}^{-\frac{\alpha_{i j}}{\sigma}}},
$$

which we will use in the rest of the paper. Finally, we remark that if the economy faces a vector of distortions $\tau$, then the formula for the probability of industry $i$ choosing industry $j$ as a supplier becomes

$$
\operatorname{Pr}\left(j \in S_{i} \mid P\right)=\frac{\left(P_{j}(1+\tau)\right)^{-\frac{\alpha_{i j}}{\sigma}}}{1+\left(P_{j}(1+\tau)\right)^{-\frac{\alpha_{i j}}{\sigma}}} .
$$

\subsection{The Distribution of Indegrees and Outdegrees in Large Networks}

We now proceed to characterize the indegree and outdegree of large networks. Let $\{\mathcal{E}(n)\}_{n=1}^{\infty}$ be a sequence of economies where $\mathcal{E}(n)$ has $n$ industries, and let $S(n)$ be the equilibrium network in economy $\mathcal{E}(n)$. Let $\mathcal{I}_{i}(n)=\frac{1}{n} \sum_{j=1}^{n} \alpha_{i j}(S(n))$ be the (normalized) indegree of industry $i$ in economy $\mathcal{E}(n)$ (meaning that it is normalized by the number of industries in the economy, $n$ ), and let $\mathcal{I}(n)=$ $\left\{\mathcal{I}_{i}(n)\right\}_{i=1}^{n}$ be the sequence of (normalized) indegrees. Analogously, let $\mathcal{O}_{j}(n)=\frac{1}{n} \sum_{i=1}^{n} \alpha_{i j}(S(n))$ be the (normalized) outdegree of industry $j$ and let $\mathcal{O}(n)=\left\{\mathcal{O}_{j}(n)\right\}_{j=1}^{n}$ be the sequence of (normalized) outdegrees. ${ }^{22}$ Both $\mathcal{I}(n)$ and $\mathcal{O}(n)$ are random variables over $\mathbb{R}^{n}$, where randomness comes from the fact that $\left\{a_{i}\left(S_{i}\right)\right\}_{i, S_{i}}$ is a sequence of random variables. Furthermore, for every $i \in \mathcal{N}$, we have $\mathcal{I}_{i}(n), \mathcal{O}(n) \leq 1$, so $\mathcal{I}(n)$ and $\mathcal{O}(n)$ can be interpreted as elements of $\ell^{\infty}$ (with $\mathcal{I}_{i}(n)=\mathcal{O}_{i}(n)=0$ for all $i>n)$.

\footnotetext{
${ }^{22}$ To simplify the terminology, we refer to $\mathcal{I}(n)$ and $\mathcal{O}(n)$ as sequences of indegrees and outdegrees, rather than normalized indegrees and normalized outdegrees. Clearly, indegrees and outdegrees can be obtained by multiplying $\mathcal{I}(n)$ and $\mathcal{O}(n)$ by $n$.
} 
The main result in this section, established in Theorem 8, is that the distribution of indegrees $\mathcal{I}(n)$ converges uniformly to the sequence $(0,0,0, \ldots) \in \ell^{\infty}$ almost surely, while the limsup and liminf of the sequence $\mathcal{O}(n)$ of outdegrees converge to non-degenerate distributions over $\ell^{\infty}$, which together imply that $\mathcal{O}(n)$ cannot converge to a non-degenerate distribution. To prove convergence in the first part of the theorem, we introduce the following strengthening of Assumption 3.

Assumption $3^{\prime}$ Suppose that Assumption 3 holds. In addition, for every industry $j$, the limit $\lim _{n \rightarrow \infty} \frac{1}{n} \sum_{i=1}^{n} \alpha_{i j}$ of average exogenous outdegrees always exists.

In what follows, we use the notation $\alpha_{j}=\lim _{n \rightarrow \infty} \frac{1}{n} \sum_{i=1}^{n} \alpha_{i j}$ and $\alpha=\left\{\alpha_{j}\right\}_{j \in \mathbb{N}}$.

Theorem 8 Suppose Assumptions 1', 2', and 3' hold. Then:

1. $\mathcal{I}(n)$ converges uniformly and almost surely to a degenerate distribution at $0 \in \ell^{\infty}$.

2. $\overline{\mathcal{O}}=\lim \sup _{n \rightarrow \infty} \mathcal{O}(n)$ is a non-degenerate distribution satisfying $\overline{\mathcal{O}} \leq \alpha$.

3. $\underline{\mathcal{O}}=\liminf _{n \rightarrow \infty} \mathcal{O}(n)$ is a non-degenerate distribution satisfying $\underline{\mathcal{O}} \geq \frac{\alpha}{2}$.

Theorem 8 shows that the distribution of outdegrees will be much more unequal than the distribution of indegrees. This is consistent with the properties of the US input-output tables, for example, as documented in Acemoglu et al. (2012). In fact, Acemoglu et al. (2012) show that the distribution of outdegrees can be approximated by a power law distribution (or put differently, it has Pareto tails). The next result is a direct corollary of this theorem and shows that if the distribution of $\alpha_{i j}$ 's can be approximated by a power law distribution, then so can the distribution of outdegrees. For this result, we utilize a simplified version of the definition of power law distribution used in Acemoglu et al. (2012).

Corollary 4 Suppose in addition that $\alpha_{i j}$ 's have a power law distribution in the sense that $\alpha_{j}$ 's in Assumption 3' satisfy $\alpha_{j}=B j^{-\delta} h(j)$, where $\delta>1, h(j)$ is a function satisfying $\lim _{x \rightarrow \infty} h(x) x^{\nu}=\infty$ and $\lim _{x \rightarrow \infty} h(x) x^{-\nu}=0$ for all $\nu>0$, and $B>0$ is such that $\sum_{j=1}^{n} \alpha_{j}<1$. Then $\mathcal{O}_{j}$ has a power law distribution. In particular,

$$
\frac{B j^{-\delta} h(j)}{2} \leq \underline{\mathcal{O}_{j}} \leq \overline{\mathcal{O}_{j}} \leq B j^{-\delta} h(j)
$$

In other words, this corollary shows that if $\alpha_{i j}$ 's have a power law distribution, then the distribution of (normalized) outdegrees is sandwiched between two power law distributions and thus has a power law distribution as well. 


\section{$7 \quad$ Identification and Estimation}

In this section, we first show that under Assumptions $1^{\prime}, 2^{\prime}$ and $3^{\prime}$, the structural parameters of our model can be identified from observed input-output linkages. We then develop an algorithm for estimating these parameters, and apply it to the US input-output tables. Finally, using the estimates of the structural parameters and estimates of the extent of distortions in the use of inputs in some selected countries, we attempt to gauge the extent to which our model generates sizable differences in the density of input-output linkages across countries and what the implications of these induced differences in input-output architecture are for GDP.

\subsection{Identification}

Under Assumptions 1', 2', and $3^{\prime}$, the parameters of our economy are given by the exogenous input-output matrix $\left\{\alpha_{i j}\right\}_{i, j \in \mathbb{N}}$, the household preference vector $\left\{\beta_{j}\right\}_{j \in \mathbb{N}}$, and the variance $\sigma$ of the productivity distribution. However, for input-output linkages that are not formed, the relevant $\alpha_{i j}$ is unobserved, and thus has to be treated as a latent variable. Nevertheless, under Assumption $3^{\prime}$, we will show that as $n \rightarrow \infty$, the vector of average exogenous outdegrees $\left\{\alpha_{j}\right\}_{j \in \mathbb{N}}$ (where $\left.\alpha_{j}=\lim _{n \rightarrow \infty} \frac{1}{n} \sum_{i=1}^{n} \alpha_{i j}\right)$ is identified when we observe prices, consumption shares, GDP growth and the structure of the realized input-output network.

To develop this point more formally, we next define our notion of identification.

Definition 4 Suppose that Assumptions $1^{\prime}, \mathscr{2}^{\prime}$, and $\mathcal{3}^{\prime}$ hold, and let $\{\mathcal{E}(n)\}_{n=1}^{\infty}$ be a sequence of economies, where $\mathcal{E}(n)$ denotes an economy with $n$ industries. Then a parameter vector $\zeta$ (where in our case $\zeta \in\left\{\alpha=\left\{\alpha_{j}\right\}_{j \in \mathbb{N}},\left\{\beta_{j}\right\}_{j \in \mathbb{N}}, \sigma\right\}$ if there exist an estimator $\widehat{\zeta}(\mathcal{E}(n))$ such that

$$
\operatorname{plim}_{n \rightarrow \infty} \widehat{\zeta}(\mathcal{E}(n))=\zeta
$$

The next theorem shows that when prices, consumption shares, the growth rate and the realized equilibrium network are observed, our key parameters are identified.

Theorem 9 Suppose that Assumptions 1', 2', and $3^{\prime}$ hold and prices, consumption shares, the growth rate, and the realized equilibrium network are observed. Then the parameters $\alpha, \beta$, and $\sigma$ are identified.

The proof of the theorem also establishes that the following are consistent estimators:

1. $\widehat{\beta}=\left(c s_{i}\right)_{i=1}^{n}$, where $c s_{i}$ denotes the consumption share of industry $i$;

2. $\widehat{\sigma}=-\frac{\pi(n)}{n \log 2 \sum_{i, j=1}^{n} \beta_{i} \mathcal{L}_{i j}}$, where $\pi(n)=\beta^{\prime} p(n)$ and $\mathcal{L}_{i j}$ denotes the $i j$-th element of the realized Leontief inverse matrix; 
3.

$$
\widehat{\alpha}_{j}=\arg \min _{z \geq 0} \frac{1}{n} \sum_{i=1}^{n}\left(\alpha_{i j}(S(n))-z P_{j}(n) \frac{P_{j}(n)^{-\frac{z}{\sigma}}}{1+P_{j}(n)^{-\frac{z}{\sigma}}}\right)^{2} .
$$

\subsection{Estimates from the US Input-Output Data}

In this subsection, we estimate the parameters of our model using the US input-output data for 2007. We use the detailed commodity-by-commodity tables from the Bureau of Economic Analysis. We follow Acemoglu et al. (2012) and exclude sectors related to government and housing. This leaves us with input-output linkages between 377 industries. We then apply the estimators described in the previous subsection to these data. Once we have estimated the average exogenous outdegree $\widehat{\alpha}_{j}$ and $\widehat{\sigma}$, we use the formulae ${ }^{23}$

$$
\widehat{P}_{j}=e^{-n \widehat{\sigma} \log (2) \sum_{i=1}^{n} \mathcal{L}_{i j}} \text { and } \widehat{\alpha}_{j}(S(n))=\widehat{\alpha}_{j} \cdot \frac{\widehat{P}_{j}^{-\frac{\widehat{\alpha}_{j}}{\widehat{\sigma}}}}{1+\widehat{P}_{j}^{-\frac{\widehat{\alpha}_{j}}{\widehat{\sigma}}}}
$$

to predict values for endogenous average outdegrees (meaning the average outdegree of industry $j$ in the observed production network $S(n)$ where some of the other industries will not have chosen industry $j$ as a supplier).

Figure 2 presents the estimated and actual distributions of average endogenous outdegrees, i.e., $\widehat{\alpha}_{j}(S(n))$ and $\alpha_{j}(S(n))=\frac{1}{n} \sum_{i=1}^{n} \alpha_{i j}(S(n))$. The two distributions are very similar: the overall average of outdegrees in the estimated and actual distributions $\left(\frac{1}{n} \sum_{j=1}^{n} \widehat{\alpha}_{j}(S(n))\right.$ and $\left.\frac{1}{n} \sum_{j=1}^{n} \alpha_{j}(S(n))\right)$ are the same, 0.0014, while the sum of outdegrees in both the estimated and actual data is approximately 0.55. A Kolmogorov-Smirnov test fails to reject the null hypothesis that the distributions of estimated and actual outdegrees are the same with a $p$-value of 0.105 .

\subsection{Counterfactual Analysis}

In this subsection, we investigate how reasonable-sized distortions and restrictions on input-output linkages impact the endogenous production network and the implications of this change in inputoutput linkages on GDP. For this purpose, we use data on sectoral taxes and input-output linkages from the OECD, which are available at the level of 34 industries and for 63 countries. For marginal distortions, we consider four specifications. We start with a marginal distortion rate of $\tau=1.4 \%$ on all industries, which is the average sectoral tax rate we observe across developing countries in the OECD data (compared to $0.26 \%$ in the United States). ${ }^{24}$ We then experiment with a specifiation in

\footnotetext{
${ }^{23}$ The formula for $\widehat{P}_{j}$ follows from Theorem 7 , as described more explicitly at the end of this subsection.

${ }^{24}$ We use the IMF's World Economic Outlook (IMF 2015) to categorize countries into three groups: More Developed Countries (MDC), Graduated Developing Countries (GDC) and Less Developed Countries (LDC). We refer to the countries in the Less Developed Countries group as developing countries. In the OECD data, these are Argentina, Bulgaria, Brazil, Cambodia, Chile, China, Colombia, Costa Rica, Croatia, Hungary, India, Indonesia, Malaysia, Mexico, Philippines, Poland, Russia, Saudi Arabia, South Africa, Thailand, Tunisia, Turkey and Vietnam.
} 


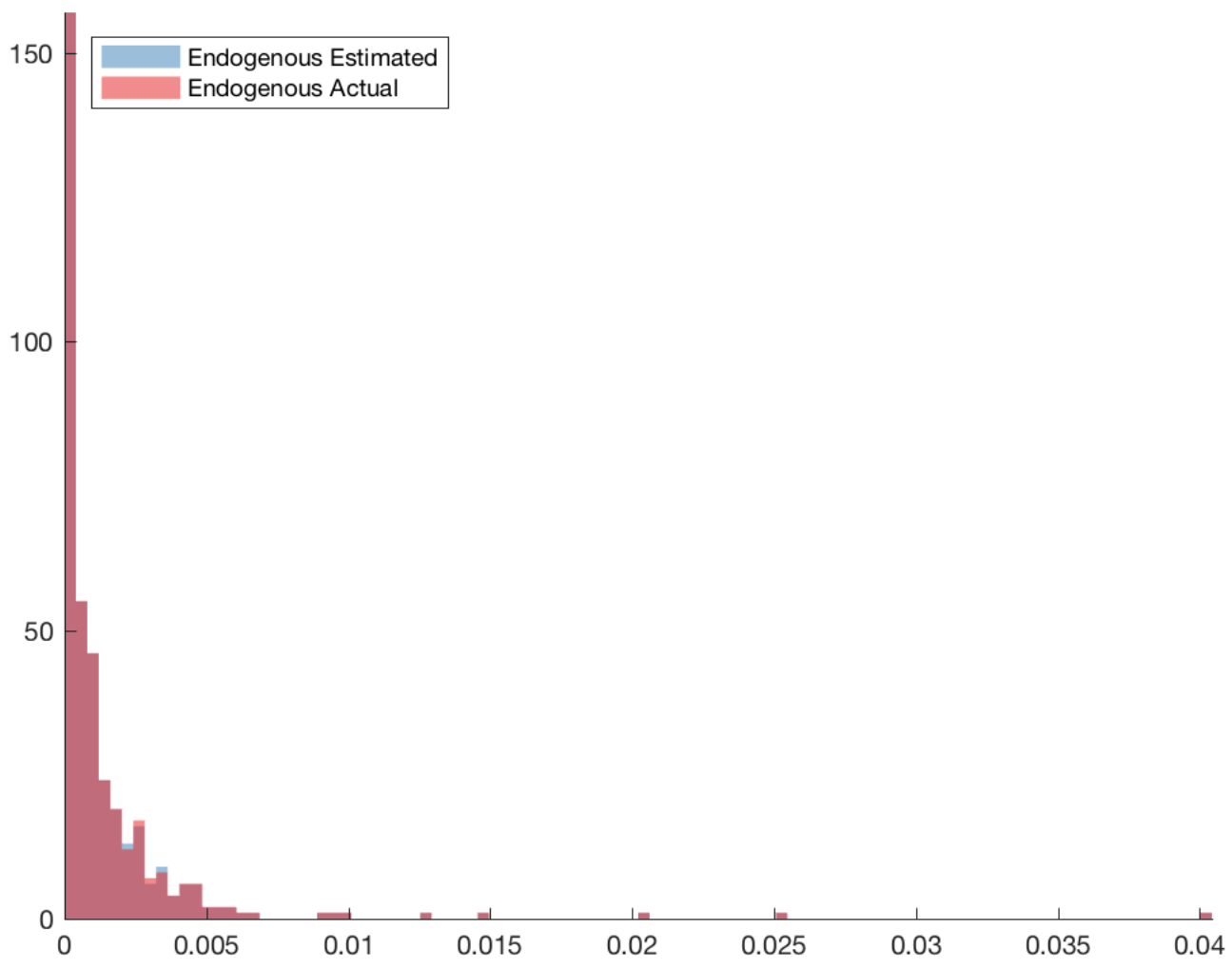

Figure 2: Distribution of estimated and actual average outdegrees. Estimates are computed from the $377 \times 377$ USA detailed input-output matrix provided by the BEA, with sectors related to government and housing removed. The red bars represent the actual sectoral average outdegrees (from US Input-Output Detail Level data from 2007) and the blue bars represent the estimated sectoral average outdegrees. The overall average outdegree (the average of observed $\alpha_{i j}$ 's) both in the data and in our estimates is 0.0014, and the average of sectoral outdegrees (the average of observed $\sum_{i} \alpha_{i j}$ 's) is 0.545 in the BEA data and 0.546 in our estimates. A Kolmogorov-Smirnov test fails to reject the null hypothesis that the distributions of estimated and actual outdegrees are the same with a $p$-value of 0.105 . 
which marginal distortions across industries are distributed normally with mean $1.4 \%$ and standard deviation $1.6 \%$, which is the average standard deviation of sectoral tax rates across developing countries in the OECD data. We also experimented with a marginal distortion rate of $\tau=10 \%$ to illustrate the implications of much higher marginal distortions (with or without the normal distribution of sectoral distortions around this value).

We then use the OECD input-output data in order to estimate the probability with which a given input-output link in the United States may be directly missing (due to institutional or other restrictions) in developing countries. The procedure for this estimation is described in Appendix $\mathrm{D}$, and leads to an estimate of $\rho \simeq 11 \%$ for the average developing country and $\rho \simeq 23 \%$ for developing countries excluding formerly communist nations. ${ }^{25}$ Similar to our counterfactual exercises for marginal distortions, we use these mean estimates assuming either no variation across sectors or a normal distribution for the probability that a link that is present in the US input-output is missing with a standard deviation of $89.5 \%$, which is the average standard deviation of the observed probabilities across developing economies in our sample.

We compute counterfactual input-output matrices and GDP levels using, separately, the abovedescribed marginal distortions and direct restrictions. The algorithm for computing these counterfactuals (described below) relies on equilibrium log productivity terms, the $a_{i}\left(S^{*}\right)$ 's, and log prices, the $p_{i}^{*}$ 's, neither of which are directly observed in the BEA data. Nevertheless, we can use Theorem 7 and Corollary 3 to obtain the asymptotic values of these variables (to which they converge almost surely as $n$ grows large). In particular, from Theorem 7 we have

$$
\lim _{n \rightarrow \infty}-\frac{p_{i}^{*}(n)}{n}=D \sum_{j=1}^{n} \mathcal{L}_{i j} \text { almost surely, }
$$

and in the proof of this theorem in Appendix A, we also establish

$$
\lim _{n \rightarrow \infty} \frac{a_{i}\left(S^{*}(n)\right)}{n}=D \text { almost surely. }
$$

Under Assumption $2^{\prime}$, we have that $D=\sigma \log 2$. Thus, an estimate $\widehat{\sigma}$ for the parameter $\sigma$ will yield an estimate $\widehat{D}=\widehat{\sigma} \log 2$ which we can use to predict log prices as $\widehat{p}_{i}^{*}(n)=-n \widehat{D} \sum_{j=1}^{n} \mathcal{L}_{i j}$ and $\log$ productivities as $\widehat{a}_{i}\left(S^{*}(n)\right)=n \widehat{D}$. In the presence of distortions, from Corollary 3 the corresponding predicted values are $\widehat{p}_{i}^{*}(n)=-(1-\rho) n \widehat{D} \sum_{j=1}^{n} \mathcal{L}_{i j}$ and $\widehat{a}_{i}\left(S^{*}(n)\right)=(1-\rho) n \widehat{D}$, where $\rho$ is the probability that there is a direct restriction preventing a link $(i, j)$ to be formed.

Using these formulae for productivity and prices we use the following algorithm for computing counterfactual input-output networks and GDP levels.

\footnotetext{
${ }^{25}$ This last sample (excluding Bulgaria, Cambodia, China, Croatia, Hungary, Poland, Russia and Vietnam) is relevant because formerly communist economies tend to have very dense input-output networks as a legacy of central planning, which often mandated inter-industry trade.
} 
1. Start with a marginal distortion vector $\left(\tau_{i}\right)_{i=1}^{n}$, a probability of links being assigned to $R$ (the set of restricted links) $\rho \in[0,1]$, the US input-output network $S_{U S}$, the input-output matrix $\alpha\left(S_{U S}\right)$, and the Leontief matrix $\mathcal{L}\left(S_{U S}\right)$.

2. Initialize $\alpha=\alpha\left(S_{U S}\right)$.

3. For every link $(i, j)$ such that $\alpha_{i j}\left(S_{U S}\right)>0$, set $\alpha_{i j}=\left\{\begin{array}{l}\alpha_{i j}\left(S_{U S}\right) \text { with probability } 1-\rho \\ 0 \text { with probability } \rho\end{array}\right.$. Initialize $\mathcal{L}=(I-\alpha)^{-1}$.

4. Initialize $P_{j}=e^{-\sigma \log 2 n \sum_{k} \mathcal{L}_{j k}+\sum_{k} \mathcal{L}_{j h} \alpha_{h k} \log \left(1+\tau_{k}\right)}$.

5. For any remaining edges $(i, j)$ in the network, let $\operatorname{Pr}_{i, j}^{0}=\frac{P_{j}^{-\frac{\alpha_{i j}}{\sigma}}}{1+\left(P_{j}\right)^{-\frac{\alpha_{i j}}{\sigma}}}$. Repeat the following procedure until the estimate of $\log$ GDP, $\widehat{y}$, converges:

- Compute the conditional probability $\frac{\operatorname{Pr}_{i j}}{\operatorname{Pr}_{i j}^{0}}$ that edge $(i, j)$ is observed in the economy with distortions, given that it is observed in the economy without distortions, where $\operatorname{Pr}_{i j}=\frac{\left(P_{j}\left(1+\tau_{j}\right)\right)^{-\frac{\alpha_{i j}}{\sigma}}}{1+\left(P_{j}\left(1+\tau_{j}\right)\right)^{-\frac{\alpha_{i j}}{\sigma}}}$. Note that when $\tau \geq 0$, we have that $0 \leq \frac{\operatorname{Pr}_{i j}}{\operatorname{Pr}_{i j}^{0}} \leq 1$.

- Create a matrix $\alpha^{t e m p}$ such that $\alpha_{i j}^{t e m p}=\left\{\begin{array}{l}\alpha_{i j} \text { with probability } \frac{\operatorname{Pr}_{i j}}{\operatorname{Pr}_{i j}^{0}} \\ 0 \text { with probability } 1-\frac{\operatorname{Pr}_{i j}}{\operatorname{Pr}_{i j}^{0}} .\end{array}\right.$

- Create an endogenous Leontief matrix $\mathcal{L}^{\text {temp }}=\left(I-\alpha^{t e m p}\right)^{-1}$.

- Update log prices as $\widehat{p}_{j}=-(1-\rho) \sigma \log 2 n \sum_{k} \mathcal{L}_{j k}^{t e m p}+\sum_{k} \mathcal{L}_{j h}^{t e m p} \alpha_{h k}^{t e m p} \log \left(1+\tau_{k}\right)$.

- Estimate $\log$ GDP as $\widehat{y}=\beta^{\prime}(\widehat{p}+\log (1+\tau))$.

A variation of this algorithm, which we use in Table 2 below, allows different removal probabilities $\rho_{i j}$ for each link $(i, j)$. In this case, we cannot use Corollary 3 to derive an asymptotic formula for log prices, but we can approximate them as $\widehat{p}_{j} \simeq-\left(1-\overline{\rho_{j}}\right) \sigma \log 2 n \sum_{k} \mathcal{L}_{j k}^{\text {temp }}+\sum_{k} \mathcal{L}_{j h}^{\text {temp }} \alpha_{h k} \log \left(1+\tau_{k}\right)$, where $\overline{\rho_{j}}=\frac{1}{n} \sum_{i=1}^{n} \rho_{i j}$.

In Table 1, we show the implications of marginal distortions. The first column gives the implied counterfactual decline in GDP in our model for the indicated specification of distortions. With moderate distortions, the effect is small, ranging between $2.7 \%$ and $3.3 \%$. When we consider the higher average value $\tau_{\mu}=10 \%$, we see a larger decline of approximately $18.5 \%$. The next columns investigate whether these declines come from the direct effects of marginal distortions or the implied changes in the input-output structure of the economy. The second column considers the implied decline in GDP if we keep the input-output network unchanged and only consider the direct effects of marginal distortions. The implied reductions in GDP are very similar to those reported in column 1. Column 3 considers the converse exercise, where we let the input-output structure adjust to marginal distortions, but then compute the counterfactual GDP without taking into account the 
direct effects of these marginal distortions. Unsurprisingly in view of the results in column 2, the implied declines are now very small. The fourth column, finally, shows why this is the case. The probability that any given input-output link is dropped because of the marginal distortions is very very small, less than $0.1 \%$ in all cases. These exercises therefore show that marginal distortions have moderate effects on GDP (and productivity), and very small effects on the equilibrium input-output linkages at our estimated parameter values. ${ }^{26}$

Table 2 then turns to an investigation of the implications of direct regulations on the production network modeled as the removal of some of the existing links in the US input-output data. We now estimate fairly large effects on GDP. The implied declines in GDP are over $80 \%$ in all cases. These large declines reflect moderate declines in the entries of the Leontief inverse matrix, since counterfactual GDP in this case is $\exp \left(\beta^{\prime} \mathcal{L}(S) a(S)-\beta^{\prime} \mathcal{L}\left(S_{U S}\right) a\left(S_{U S}\right)\right)$. These numbers suggest that the potential productivity implications of changes in the input-output architecture of an economy resulting from direct restrictions could be much larger than those resulting from marginal distortions. ${ }^{27}$

\section{Conclusion}

There are marked differences across countries in how production is organized. Richer and more productive countries appear to have denser production networks - where each disaggregated industry uses the output of several other industries as inputs. We develop a tractable model of endogenous production networks to provide a conceptual framework for understanding these patterns and how differences in distortions or technologies can translate into variation in production networks.

In our model, each product can be produced by combining labor and an endogenous subset of the other products as inputs. Different combinations of inputs generate different constant returns to scale production functions with (prespecified) levels of productivity. What makes our framework tractable is our assumption that markets are "contestable". This means, in particular, that production technologies are available to a large number of potential producers (this avoids the interesting but challenging issues that would be associated with differential market power of firms that make investments in different types of technologies and issues of bilateral monopoly between suppliers and customers of inputs).

Using this setup, we establish the existence and uniqueness of an equilibrium with an endogenous

\footnotetext{
${ }^{26}$ Specifically, the counterfactual decline in GDP without a change in the input-output network is computed as $\exp \left(\beta^{\prime} \mathcal{L}\left(S_{U S}\right)\left(a\left(S_{U S}\right)+\log (1+\tau)\right)-\beta^{\prime} \mathcal{L}\left(S_{U S}\right) a\left(S_{U S}\right)\right)$, while the expression for the counterfactual decline in GDP resulting from the change in the input-output network without taking the direct impact of marginal distortions into account is $\exp \left(\beta^{\prime} \mathcal{L}(S) a(S)-\beta^{\prime} \mathcal{L}\left(S_{U S}\right) a\left(S_{U S}\right)\right)$. In our calculations, we use the asymptotic approximation $a_{i}(S) \simeq$ $a_{i}\left(S_{U S}\right) \simeq n \sigma \log 2$. Similarly, with probability $\rho$ for direct restrictions, we use the asymptotic approximations $a_{i}(S) \simeq(1-\rho) n \sigma \log 2$ and $a_{i}\left(S_{U S}\right) \simeq n \sigma \log 2$.

${ }^{27}$ It is also interesting to note that the implied declines in GDP from the implied probabilities of missing inputoutput links are in the ballpark of the observed gap between the average developing country in our sample (both with and without the formerly-communist nations) and the United States, which is about $72 \%$.
} 


\section{Table 1: Counterfactual Analysis With Marginal Distortions}

\author{
Distribution of \\ Marginal Distortions $\tau$
}

Counterfactual GDP

$(2)$
Probability that an edge in US network is missing

(1)

(3)

\begin{tabular}{cccccc} 
Mean & Standard Deviation & Model & Constant IO Matrix & Constant Taxes & \\
\hline \hline $1.4 \%$ & 0 & $-2.7 \%$ & $-2.7 \%$ & $-0 \%$ & $<0.01 \%$ \\
$1.4 \%$ & $1.6 \%$ & $-3.3 \%$ & $-3.3 \%$ & $-0 \%$ & $<0.01 \%$ \\
$10 \%$ & 0 & $-18.4 \%$ & $-17.5 \%$ & $-1.1 \%$ & $<0.01 \%$ \\
$10 \%$ & $1.6 \%$ & $-18.5 \%$ & $-17.5 \%$ & $-1.3 \%$ & $<0.01 \%$
\end{tabular}

Note: This table reports the results of a counterfactual exercise where we impose a marginal distortion $1+\tau_{i}$ on prices so that if a firm's unit cost is given by $P_{i}$, the price of its product is $P_{i}\left(1+\tau_{i}\right)$. Imposing this marginal distortion has two effects on GDP: a direct effect from the fact that input prices are higher, and an indirect effect by changing the input-output matrix. The marginal distortions $\tau_{i}$ are assumed to be drawn from a truncated normal distribution with location and scale parameters $\mu$ and $\sigma$. The table rows correspond to different levels of $\mu$ and $\sigma$. Column (1) reports the counterfactual GDP decline, $\exp \left(\beta^{\prime} \mathcal{L}\left(S^{*}\right)\left(a\left(S^{*}\right)-\log (1+\tau)\right)-\beta^{\prime} \mathcal{L}\left(S_{U S}\right) a\left(S_{U S}\right)\right)$. Column (2) reports the counterfactual GDP decline, $\exp \left(\beta^{\prime} \mathcal{L}\left(S^{*}\right) a\left(S^{*}\right)-\beta^{\prime} \mathcal{L}\left(S_{U S}\right) a\left(S_{U S}\right)\right)$, capturing only the indirect effect from the change in $\mathcal{L}$. Column (3) reports the counterfactual GDP decline, $\exp \left(\beta^{\prime} \mathcal{L}\left(S_{U S}\right)\left(a\left(S^{*}\right)-\log (1+\tau)\right)-\beta^{\prime} \mathcal{L}_{\mathcal{U S}} a\left(S_{U S}\right)\right)$, capturing only the direct effect of imposing marginal distortions, while fixing the input-output matrix to be the observed BEA input-output matrix for the US. Column (4) provides the probabilities that an edge present in the US input-output network is missing with the indicated distortions.

Table 2: Counterfactual Analysis With Direct Restrictions

Distribution of Restriction Probability $\rho \quad$ Counterfactual GDP

\begin{tabular}{ccc} 
Mean & Standard Deviation & \\
\hline \hline $10.9 \%$ & 0 & $-80.5 \%$ \\
$10.9 \%$ & $89.5 \%$ & $-89.5 \%$ \\
$23.8 \%$ & 0 & $-95.8 \%$ \\
$23.8 \%$ & $89.5 \%$ & $-95.8 \%$
\end{tabular}

Note: This table reports the results of a counterfactual exercise where edges are removed from the observed US input output graph with some probability $\rho$, which is drawn from a truncated normal distribution with parameters $\mu$ and $\sigma$. The table rows correspond to different levels of $\mu$ and $\sigma$. The drop in counterfactual GDP is given by $\exp \left(\beta^{\prime} \mathcal{L} a\left(S^{*}\right)-\beta^{\prime} \mathcal{L}\left(S_{U S}\right) a\left(S_{U S}\right)\right)$. 
production network. We then use our framework to clarify several new economic trade-offs and comparative statics that arise in the context of endogenous production networks. Namely:

- when a product adopts additional inputs to minimize its costs, this not only reduces its price, but (weakly) reduces all prices in the economy. This "complementarity" is a consequence of the fact that with the new technology, this product has now become cheaper and thus a more attractive input to all others, enabling them to reduce costs and prices as well;

- under a reasonable assumption that ensures that lower prices do not discourage technology adoption, a change in technology that makes the adoption of additional inputs more productive for one industry expands technology sets for all industries. This second dimension of complementarity is a new feature of environments with endogenous production networks;

- the technology comparative statics mentioned in the previous bullet point are potentially "discontinuous" in the sense that a small change for a single industry can trigger a chain reaction, leading to major shifts in the production structure of many industries;

- both marginal distortions (e.g., taxes) that increase the prices of certain inputs and direct restrictions on input-output links increase equilibrium prices, decrease the density of the production network, and reduce GDP. The full effects of these distortions extend beyond the affected sectors because those that experience higher marginal distortions or are restricted from using valuable inputs become more expensive themselves and are thus less likely to be used by others, spreading the effects of distortions throughout the production network of the economy.

The second part of the paper uses a dynamic version of our framework to study the growth implications of the endogenous evolution of the production network. Our main result from this analysis is that the endogenous selection of input suppliers and the indirect effects that this creates on the equilibrium structure of the production network emerge as powerful forces towards sustained economic growth. The origin of sustained growth in our model is related to, but different, from Weitzman's idea of recombinant growth. When a new product arrives, it becomes a potential input for all existing products, and significantly expands the number of input combinations (production techniques) available to other industries. Namely, when there are $n$ products, the arrival of one more new product increases the combinations of inputs that each existing product can use from $2^{n-1}$ to $2^{n}$, thus enabling nontrivial cost reductions from the choice of optimal technology combinations. A first impetus for growth comes from this expanded set of techniques to which firms have access, and this part of our mechanism is related to Weitzman (1998), even if formulated differently from his in Weitzman's approach, ideas can be recombined without limit, whereas here there is an explicit focus on the new combinations of inputs that are made possible by the addition of one more product 
into the set of possible inputs. Growth in our economy is not driven by this first impetus alone, however. It is undergirded by the fact that the adoption of a new production technique reduces the price of the relevant product, encouraging other industries to adopt this product as an additional input and change their production techniques. To highlight the role of these new elements, we document that growth in our model does not follow if firms do not make endogenous choices of input suppliers, that there is no contribution to growth from expanding product variety (because we impose that the value of new products in consumption is essentially zero), and that there are sizable indirect effects on growth coming from the cost reduction benefits to other industries.

The third part of the paper derives the empirical implications of our framework and makes a first attempt at confronting them with data. We first derive a closed-form expression for the likelihood of a sector being adopted as an input supplier to another sector. Second, we establish that under fairly mild assumptions, outdegrees (how often a sector is being used as a supplier to other industries) are much more unequally distributed than indegrees (how many inputs a sector is using). This prediction is consistent with the patterns of input-output linkages in the US economy. Finally, we show how the underlying parameters of our model can be estimated structurally, and carry out such an estimation using the disaggregated US input-output tables. We then use these estimates to gauge the implications of reasonably-sized distortions on the density of the input-output network and aggregate productivity.

We view our paper as a first step in the analysis of endogenous formation of production networks. As mentioned above, our analysis was greatly simplified by the "contestability" assumption. Though this was a very useful starting point, it precludes a systematic investigation of endogenous production networks at finer levels of disaggregation - e.g., at the firm level rather than at the level of disaggregated industries — where contestability would make less sense. Relaxing this assumption would enable an analysis of relationship-specific investments taking place at the same time as the choice of input suppliers, significantly enriching the theoretical framework and its empirical reach. Second, we illustrated the empirical applications of this framework only briefly. A more in-depth structural exercise estimating the parameters regulating the endogenous evolution of production networks and counterfactual analysis based on these parameters are obvious fruitful directions for future research. Beyond firm-level datasets with information on flows of intermediate goods, detailed bilateral international trade flows would be another empirical domain where a similar approach could be developed. Finally, the linkages between the evolution of the production network and long-run economic growth are another promising area that calls for more work. Introducing endogenous innovation and entry and exit of firms into this framework is a challenging but a necessary step to obtain a more satisfactory picture of the co-determination of the production network and endogenous technology choices, and their impact on growth. 


\section{Appendix A: Omitted Proofs From the Text}

Proof of Lemma 1. First, suppose that $P^{*}$ is a vector of equilibrium prices. Then $P_{i}^{*}=$ $K_{i}\left(S_{i}, A_{i}, P^{*}\right)$ follows from the second condition of the definition of equilibrium (firm optimization).

To prove the other direction, suppose that $P_{i}^{*}=K_{i}\left(S_{i}, A_{i}, P^{*}\right)$. We show that $P$ supports an equilibrium. Let $X_{i}^{*}$ and $L_{i}^{*}$ be the optimal solutions to the firm's cost minimization problem (3) given $P^{*}$

Let $C^{*}$ be the consumer's optimal choice of good $i$ when facing the vector of prices $P^{*}$. Note that the assumption that $P_{i}^{*}>0$ ensures $X_{i}^{*}$ and $C_{i}^{*}$ are finite.

For given $P^{*}$, let $x_{i j}$ denote the units of good $j$ used for producing one unit of good $i$, which depends on $P^{*}$, but due to constant returns to scale, is independent of $Y_{i}$. Similarly let $l_{i}$ be the unit labor requirement of good $i$. These quantities are uniquely determined by the strict quasi-concavity of the production function imposed in Assumption 1. Then $X_{i j}^{*}=x_{i j} Y_{i}^{*}$ and $L_{i}^{*}=l_{i} Y_{i}^{*}$. Then market clearing implies

$$
C_{j}^{*}+\sum_{i=1}^{n} x_{i j} Y_{i}^{*}=Y_{j}^{*}
$$

Multiplying this equation by $P_{j}^{*}$,

$$
P_{j}^{*} C_{j}^{*}+\sum_{i=1}^{n} \frac{P_{j}^{*} x_{i j}}{P_{i}^{*}} P_{i}^{*} Y_{i}^{*}=P_{j}^{*} Y_{j}^{*},
$$

or

$$
\widehat{C}_{j}^{*}+\sum_{i=1}^{n} \frac{P_{j}^{*} x_{i j}}{P_{i}^{*}} \widehat{Y}_{i}^{*}=\widehat{Y}_{j}^{*},
$$

where "^" denotes a nominal variable. Denoting the matrix of $\frac{P_{j}^{*} x_{i j}}{P_{i}^{*}}$ 's by $\bar{X}$, this nominal version of the market clearing condition can be expressed as $\left(I-\bar{X}^{\prime}\right) \widehat{Y}^{*}=\widehat{C}^{*}$. This is a system of $n$ equations in $n$ unknowns, the nominal sectoral outputs, $\widehat{Y}_{1}, \ldots, \widehat{Y}_{n}$. As long as $\operatorname{det}\left(I-\bar{X}^{\prime}\right) \neq 0$, this system of equation admits a unique solution $\widehat{Y}_{1}^{*}, \ldots, \widehat{Y}_{n}^{*}$. Since we are taking the vector of prices $P^{*}$ as given, this solution translates into a unique vector of sectoral outputs $Y_{1}^{*}, \ldots, Y_{n}^{*}$. Clearly, $\operatorname{det}\left(I-\bar{X}^{\prime}\right) \neq 0$ if and only if 1 is not an eigenvalue of $\bar{X}^{\prime}$. From the fourth condition of the definition of equilibrium, $P_{i}^{*}=K_{i}\left(S_{i}, A_{i}, P^{*}\right)$, which can be equivalently written as $\sum_{i=1}^{n} P_{j}^{*} x_{i j}+l_{i}=P_{i}^{*}$. Because labor is an essential factor of production (cfr. Assumption 1), the elements of $\bar{X}^{\prime}$ are between 0 and 1 and strictly less than 1. But then the Perron-Frobenius theorem implies that $\bar{X}^{\prime}$ has a positive largest eigenvalue that is strictly less than 1 , and sectoral outputs, and thus consumption and intermediate input levels, are uniquely determined. Moreover, $L_{i}^{*}=l_{i} Y_{i}^{*}$ is also uniquely determined for each $i$. This completes the proof that $P^{*}>0$ can support an equilibrium if $P_{i}^{*}=K_{i}\left(S_{i}, A_{i}, P^{*}\right)$.

Proof of Theorem 1. Let $\kappa(P)=\left(\min _{S_{1}} K_{1}\left(S_{1}, A_{1}\left(S_{1}\right), P\right), \ldots, \min _{S_{n}} K_{n}\left(S_{n}, A_{n}\left(S_{n}\right), P\right)\right)$. We 
first show that $\kappa$ has a fixed point, and then show that this corresponds to an equilibrium. To do this, we prove the following lemma as an intermediate step.

Lemma A1 Let $\mathbb{L}=\left\{P \geq 0: P_{i}=\min _{S_{i}} K_{i}\left(S_{i}, A_{i}\left(S_{i}\right), P\right)\right\}$. Then $\mathbb{L}$ is a non-empty complete lattice with respect to the operations $P \wedge Q=\left(\min \left(P_{1}, Q_{1}\right), \ldots, \min \left(P_{n}, Q_{n}\right)\right), P \vee Q=$ $\left(\max \left(P_{1}, Q_{1}\right), \ldots, \max \left(P_{n}, Q_{n}\right)\right)$.

Proof of Lemma A1. Let $\mathbb{O}=\left\{\left(x_{1}, \ldots, x_{n}\right): x_{i} \geq 0\right\}$, and then by definition $\kappa: \mathbb{O} \rightarrow \mathbb{O}$. We will first show that there is a subset $\widetilde{\mathbb{O}} \subset \mathbb{O}$ which is a complete lattice with respect to $\wedge$ and $\vee$, and then establish that that $\kappa(P)$ is increasing in $P$ and maps $\widetilde{\mathbb{O}}$ to $\widetilde{\mathbb{O}}$. The result that $\mathbb{L}$ is a complete lattice follows from these two steps.

To establish the first step, note that for any $i$, we can produce good $i$ using only labor and incur a cost $\overline{P_{i}}=K_{i}\left(\varnothing, A_{i}(\varnothing),\left\{P_{j}\right\}_{j \in \varnothing}\right)$ that does not depend on the price vector $P$. Thus, we have $\kappa(P) \leq\left(\overline{P_{1}}, \ldots, \overline{P_{n}}\right)$ for all price vectors $P$. Since labor is essential in production, we have $K_{i}\left(S_{i}, A_{i}\left(S_{i}\right), 0\right)>0$ for every set $S_{i}$. Define $\underline{P_{i}}=\kappa_{i}(0)=\min _{S_{i}} K_{i}\left(S_{i}, A_{i}\left(S_{i}\right), 0\right)$. Since $K_{i}$ is increasing in price, we have $\kappa(P) \geq \kappa(0)=\left(\underline{P_{1}}, \ldots, \underline{P_{n}}\right)$ for every price vector $P$. Then $\widetilde{\mathbb{O}}=$ $\times_{i=1}^{n}\left[\underline{P_{i}}, \overline{P_{i}}\right]$ is a complete lattice, and $\kappa$ maps $\widetilde{\mathbb{O}}$ to $\widetilde{\mathbb{O}}$.

The second step is immediate from the definition of $\kappa(P)$. If $P^{\prime} \leq P$, then for any $i$ and $S_{i}$, we have $K_{i}\left(S_{i}, A_{i}\left(S_{i}\right), P^{\prime}\right) \leq K_{i}\left(S_{i}, A_{i}\left(S_{i}\right), P\right)$. Taking minima on both sides, we get $\min _{S_{i}} K_{i}\left(S_{i}, A_{i}\left(S_{i}\right), P\right) \leq \min _{S_{i}} K_{i}\left(S_{i}, A_{i}\left(S_{i}\right), P^{\prime}\right)$, so $\kappa\left(P^{\prime}\right) \leq \kappa(P)$. We conclude from Tarski's fixed point theorem that $\mathbb{L}$ is a non-empty complete lattice.

Since $\mathbb{L}$ is a non-empty complete lattice, $\kappa$ has a fixed point, and in fact, a smallest fixed point. Take this smallest fixed point, which simultaneously satisfies $P_{i}^{*}=K_{i}\left(S_{i}^{*}, A_{i}\left(S_{i}^{*}\right), P^{*}\right)$ and $S_{i}^{*} \in \arg \min _{S_{i}} K_{i}\left(S_{i}, A_{i}\left(S_{i}\right), P^{*}\right)$. That is, given $P^{*}$, technology choice $S_{i}^{*}$ is optimal, and given $S^{*}$, firms minimize costs. Then with the same argument as in Lemma 1, there exist equilibrium quantities $X^{*}, L^{*}$ and $C^{*}$, and $P^{*}$ is minimal by construction, and thus $\left(P^{*}, C^{*}, S^{*}, L^{*}, X^{*}, Y^{*}\right)$ is an equilibrium.

Proof of Theorem 2. Let $P^{*}$ be the minimal element of lattice $\mathbb{L}$ defined in the proof of Theorem 1 , which is of course an equilibrium price vector. If $P^{* *}$ is another equilibrium price vector, it must be contained in $\mathbb{L}$ and therefore satisfy $P^{* *}>P^{*}$. We now derive a contradiction to $P^{* *}>P^{*}$.

First, note that for each $i \in \mathcal{N}$, the unit cost function $K_{i}\left(S_{i}, A_{i}\left(S_{i}\right), P\right)$ is concave in prices given $S_{i}$. Since the minimum of a collection of concave functions is concave, $\kappa_{i}(P)=$ $\min _{S_{i}} K_{i}\left(S_{i}, A_{i}\left(S_{i}\right), P\right)$ is also concave.

Then, let $\lambda \in(0,1)$ be such that $\lambda P^{* *} \leq P^{*}$, with at least some $r \in \mathcal{N}$ such that $\lambda P_{r}^{* *}=P_{r}^{*}$. 
We have

$$
\begin{aligned}
\kappa_{r}\left(P^{*}\right)-P_{r}^{*} & \geq \kappa_{r}\left(\lambda P^{* *}\right)-\lambda P_{r}^{* *} \\
& \geq(1-\lambda) \kappa_{r}(0)+\lambda \kappa_{r}\left(P^{* *}\right)-\lambda P_{r}^{* *} \\
& \geq(1-\lambda) \kappa_{r}(0) \\
& >0
\end{aligned}
$$

where the first line follows because $\kappa_{r}$ is nondecreasing, $\lambda P^{* *} \leq P^{*}$, and $\lambda P_{r}^{* *}=P_{r}^{*}$. The second line follows from the concavity of $\kappa_{r}$. The third line simply uses the fact that $P^{* *}$ is a fixed point, i.e., $\kappa_{r}\left(P^{* *}\right)=P_{r}^{* *}$. Finally, the last inequality follows because labor is essential by Assumption 1, which implies $\kappa_{r}(0)>0$. But this contradicts the hypothesis that $P^{*}$ is a fixed point. This contradiction establishes the uniqueness of equilibrium prices, and then the uniqueness of equilibrium allocations follows from Lemma $1 .^{28}$

To prove that the equilibrium network is generically unique, let $S^{*} \neq S^{* *}$ be two arbitrary networks and let $\mathcal{A}\left(S, S^{* *}\right)=\left\{A: S^{*}\right.$ and $S^{* *}$ are both equilibrium networks $\}$. Note that we can write $\mathcal{A}$ as the countable union $\cup_{S^{*}, S^{* *}} \mathcal{A}\left(S^{*}, S^{* *}\right)$. Thus, if we prove that $\mathcal{A}\left(S^{*}, S^{* *}\right)$ has measure zero, then we can conclude that $\mathcal{A}$ has measure zero. Define

$$
\Delta_{i}\left(S^{*}, S^{* *}, A\right)=K_{i}\left(S_{i}^{*}, A_{i}\left(S_{i}^{*}\right), P^{*}\right)-K_{i}\left(S_{i}^{* *}, A_{i}\left(S_{i}^{* *}\right), P^{*}\right),
$$

and note that for all parameters $A \in \mathcal{A}\left(S^{*}, S^{* *}\right)$ and each $i \in\{1, \ldots, n\}$, we have $\Delta_{i}\left(S^{*}, S^{* *}, A\right)=0$.

Because $S^{*} \neq S^{* *}$, there is at least one industry $i$ such that $S_{i}^{*} \neq S_{i}^{* *}$. Recall also that the cost function $K_{i}\left(S_{i}, A_{i}\left(S_{i}\right), P\right)$ is continuous and strictly decreasing in $A_{i}\left(S_{i}\right) \in \mathbb{R}^{\ell}$. Let $A_{i,-S_{i}^{*}}=\left\{A_{i}\left(S_{i}\right)\right\}_{S_{i} \neq S_{i}^{*}}$ be the vector of all technology terms for sets different than $S_{i}^{*}$ and let $A_{i,-1}\left(S_{i}^{*}\right)=\left\{A_{i, 2}\left(S_{i}^{*}\right), \ldots, A_{i, \ell}\left(S_{i}^{*}\right)\right\}$ be the vector of all components of $A_{i}\left(S_{i}^{*}\right)$ except for the first component $A_{i, 1}\left(S_{i}^{*}\right)$. If we keep $A_{i,-S_{i}^{*}}$ and $A_{i,-1}\left(S_{i}^{*}\right)$ constant, then $\Delta_{i}\left(S_{i}^{*}, S_{i}^{* *}, A\right)$ is a continuous and strictly decreasing function of one real variable $A_{i, 1}\left(S_{i}^{*}\right)$. This implies that, for any fixed $A_{i,-S_{i}^{*}}, A_{i,-1}\left(S_{i}^{*}\right)$, there exists a unique value of $A_{i, 1}\left(S_{i}^{*}\right)$ that satisfies $\Delta_{i}\left(S_{i}^{*}, S_{i}^{* *}, A\right)=0$. Hence, $\mathcal{A}\left(S^{*}, S^{* *}\right)=\left\{A: \Delta_{i}\left(S_{i}^{*}, S_{i}^{* *}, A\right)=0\right.$ for each $\left.i\right\}$ has measure zero in $\mathbb{R}^{n \times \ell \times 2^{n-1}}$, which implies that the equilibrium network is generically unique.

Proof of Lemma 2. Let $i \in \mathcal{N}$, and let $S_{i}^{\prime} \supset S_{i}, A_{i}^{\prime} \geq A_{i}$. Let $\mathcal{X}=\left(S_{i}, A_{i}^{\prime}\right), \mathcal{Y}=\left(S_{i}^{\prime}, A_{i}\right)$ and use the product lattice ordering so that $\mathcal{X} \vee \mathcal{Y}=\left(S_{i}^{\prime}, A_{i}^{\prime}\right), \mathcal{X} \wedge \mathcal{Y}=\left(S_{i}, A_{i}\right)$. Suppose that $K_{i}\left(S_{i}^{\prime}, A_{i}\left(S_{i}^{\prime}\right), P\right)-K_{i}\left(S_{i}, A_{i}\left(S_{i}\right), P\right) \leq 0$. In our lattice notation, this can be written as $K_{i}(\mathcal{Y}) \leq$ $K_{i}(\mathcal{X} \wedge \mathcal{Y})$. The quasi-submodularity of $K_{i}$ implies that $K_{i}(\mathcal{X} \vee \mathcal{Y}) \leq K_{i}(\mathcal{X})$ which is the same as writing $K_{i}\left(S_{i}^{\prime}, A_{i}^{\prime}\left(S_{i}^{\prime}\right), P\right)-K_{i}\left(S_{i}, A_{i}^{\prime}\left(S_{i}\right), P\right) \leq 0$. Thus, we conclude that

$$
K_{i}\left(S_{i}^{\prime}, A_{i}\left(S_{i}^{\prime}\right), P\right)-K_{i}\left(S_{i}, A_{i}\left(S_{i}\right), P\right) \leq 0 \Longrightarrow K_{i}\left(S_{i}^{\prime}, A_{i}^{\prime}\left(S_{i}^{\prime}\right), P\right)-K_{i}\left(S_{i}, A_{i}^{\prime}\left(S_{i}\right), P\right) \leq 0 .
$$

\footnotetext{
${ }^{28}$ This part of the proof builds on Kennan's (2001) proof of uniqueness of fixed point for a concave function.
} 
Proof of Theorem 3. Let $P^{0}=P^{*}$ and $S^{0}=S^{*}$ be the initial vector of equilibrium prices and equilibrium network. Note that $P^{0}$ satisfies the fixed-point conditions $P_{i}^{0}=$ $\min _{S_{i}} K_{i}\left(S_{i}, A\left(S_{i}\right), P^{0}\right)$ for all $i$. Suppose that $A_{i}(\cdot)$ increases to $A_{i}^{\prime}(\cdot)$, and define $P^{1}$ so that $P_{i}^{1}=\min _{S_{i}} K_{i}\left(S_{i}, A_{i}^{\prime}\left(S_{i}\right), P^{0}\right)$. Since $K_{i}$ is decreasing in $A_{i}$, we have $P_{i}^{1}=\min _{S_{i}} K_{i}\left(S_{i}, A_{i}^{\prime}\left(S_{i}\right), P^{0}\right) \leq$ $\min _{S_{i}} K_{i}\left(S_{i}, A_{i}\left(S_{i}\right), P^{0}\right)=P_{i}^{0}$, establishing that $P^{1} \leq P^{0}$.

As in the proof of Theorem 1, define $\kappa(P)=\min _{S_{i}} K_{i}\left(S_{i}, A_{i}^{\prime}\left(S_{i}\right), P\right)$. The equilibrium price $P^{* *}$ under the new productivity function $A_{i}^{\prime}$ is the minimal fixed point of $\kappa$. For $t \geq 1$, define $P^{t}=\kappa\left(P^{t-1}\right)$ and note that, since $\kappa$ is increasing in $P$ and $P^{1} \leq P^{0}$, we have $\lim _{t \rightarrow \infty} P^{t} \leq P^{1} \leq P^{0}=P^{*}$. Furthermore, since $\kappa$ is continuous, $\lim _{t \rightarrow \infty} P^{t}$ is a fixed point of $\kappa$. Since $P^{* *}$ is the minimal fixed point, we must have $P^{* *} \leq \lim _{t \rightarrow \infty} P^{t} \leq P^{0}=P^{*}$.

Proof of Theorem 4. Let $S^{0}=S^{*}$ be the initial equilibrium network. Note that $S^{0}$ satisfies the fixed-point conditions $S_{i}^{0}=\arg \min _{S_{i}} K_{i}\left(S_{i}, A\left(S_{i}\right), P^{*}\right)$ for all $i$. Suppose that the shift from $A_{i}(\cdot)$ to $A_{i}^{\prime}(\cdot)$ is a positive shock, and define $S^{1}$ such that $S_{i}^{1} \in \arg \min _{S_{i}} K_{i}\left(S_{i}, A_{i}^{\prime}\left(S_{i}\right), P^{*}\right)$. Using the definition of positive technology shock, we can apply Theorem 4 in Milgrom and Shannon (1994) to infer that $S_{i}^{0} \subset S_{i}^{1}$.

As in the proof of Theorem 1 , define $\kappa(P)=\min _{S_{i}} K_{i}\left(S_{i}, A_{i}^{\prime}\left(S_{i}\right), P\right)$. Let $P^{0}=P^{*}$ and define $P^{t}=\kappa\left(P^{t-1}\right)$ for $t \geq 1$. From the proof of Theorem 3 , we know that $P^{t}$ is a decreasing sequence with $P^{* *} \leq \lim _{t \rightarrow \infty} P^{t} \leq P^{*}$. Since $P^{* *} \leq P^{*}$, we apply once more Theorem 4 of Milgrom and Shannon (1994) to obtain $S_{i}^{* *}=\arg \min _{S_{i}} K_{i}\left(S_{i}, A_{i}^{\prime}\left(S_{i}\right), P^{* *}\right) \supset \arg \min _{S_{i}} K_{i}\left(S_{i}, A_{i}^{\prime}\left(S_{i}\right), P^{*}\right)=S_{i}^{1} \supset S_{i}^{0}=S_{i}^{*}$. We conclude that $S^{*} \subset S^{* *}$.

Proof of Proposition 1. Because $F_{i}\left(L_{i}, X_{i}, A_{i}\left(S_{i}\right), S_{i}\right)$ is supermodular, the profit function $\Pi_{i}\left(L_{i}, X_{i}, A_{i}\left(S_{i}\right), S_{i}, P\right)=F_{i}\left(L_{i}, X_{i}, A_{i}\left(S_{i}\right), S_{i}\right)-\sum_{j \in S_{i}} P_{i} X_{i j}-L_{i}$ is supermodular in $L_{i}, X_{i}, A_{i}\left(S_{i}\right), S_{i}$ and $-P$. Topkis (1998) shows that the function

$$
\widetilde{\Pi}_{i}\left(S_{i}, A_{i}\left(S_{i}\right), P\right)=\max _{X_{i}, L_{i}} \Pi_{i}\left(L_{i}, X_{i}, A_{i}\left(S_{i}\right), S_{i}, P\right)
$$

is supermodular in $A_{i}\left(S_{i}\right), S_{i},-P$. Thus, $\widetilde{\Pi}_{i}$ will satisfy the single-crossing condition $\widetilde{\Pi}_{i}\left(S_{i}^{\prime}, A_{i}\left(S_{i}^{\prime}\right), P\right) \geq \widetilde{\Pi}_{i}\left(S_{i}, A_{i}\left(S_{i}\right), P\right) \quad \Longrightarrow \quad \widetilde{\Pi}_{i}\left(S_{i}^{\prime}, A_{i}\left(S_{i}^{\prime}\right), P^{\prime}\right) \geq \widetilde{\Pi}_{i}\left(S_{i}, A_{i}\left(S_{i}\right), P^{\prime}\right)$. Let $Q_{i}(P)$ be the demand for good $i$ when the prices are $P$, and write $\widetilde{\Pi}_{i}\left(S_{i}, A_{i}\left(S_{i}\right), P\right)=Q_{i}(P)\left(P_{i}-\right.$ $\left.K_{i}\left(S_{i}, A_{i}\left(S_{i}\right), P\right)\right)$. The cost function satisfies the single-crossing condition with the following argument:

$$
\begin{gathered}
K_{i}\left(S_{i}^{\prime}, A_{i}\left(S_{i}^{\prime}\right), P\right) \leq K_{i}\left(S_{i}, A_{i}\left(S_{i}\right), P\right) \Longleftrightarrow \\
Q_{i}(P)\left(P_{i}-K_{i}\left(S_{i}^{\prime}, A_{i}\left(S_{i}^{\prime}\right), P\right)\right) \geq Q_{i}(P)\left(P_{i}-K_{i}\left(S_{i}, A_{i}\left(S_{i}\right), P\right)\right) .
\end{gathered}
$$


But the last inequality implies

$$
\begin{gathered}
Q_{i}\left(P^{\prime}\right)\left(P_{i}^{\prime}-K_{i}\left(S_{i}^{\prime}, A_{i}\left(S_{i}^{\prime}\right), P^{\prime}\right)\right) \geq Q_{i}\left(P^{\prime}\right)\left(P_{i}^{\prime}-K_{i}\left(S_{i}, A_{i}\left(S_{i}\right), P^{\prime}\right)\right) \Longleftrightarrow \\
K_{i}\left(S_{i}^{\prime}, A_{i}\left(S_{i}^{\prime}\right), P^{\prime}\right) \leq K_{i}\left(S_{i}, A_{i}\left(S_{i}\right), P^{\prime}\right),
\end{gathered}
$$

which is the desired result.

Proof of Proposition 2. Since the price single-crossing condition is preserved by monotonic transformation, it suffices to show that it is satisfied by the log unit cost function. To show that the log unit cost function satisfies the single-crossing conditions, let $S_{i} \subset S_{i}^{\prime}$ and $p^{\prime} \leq p$ and note that

$$
\begin{gathered}
k_{i}\left(S_{i}^{\prime}, a_{i}, p\right)-k_{i}\left(S_{i}, a_{i}, p\right) \leq 0 \Longleftrightarrow \\
\sum_{j \in S_{i}^{\prime}} \alpha_{i j} p_{j}-\sum_{j \in S_{i}} \alpha_{i j} p_{j}-a_{i}\left(S_{i}^{\prime}\right)+a_{i}\left(S_{i}\right) \leq 0 \Longleftrightarrow \\
\sum_{j \in S_{i}^{\prime}-S_{i}} \alpha_{i j} p_{j}-a_{i}\left(S_{i}^{\prime}\right)+a_{i}\left(S_{i}\right) \leq 0 \Longrightarrow \\
\sum_{j \in S_{i}^{\prime}-S_{i}} \alpha_{i j} p_{j}^{\prime}-a_{i}\left(S_{i}^{\prime}\right)+a_{i}\left(S_{i}\right) \leq 0 \Longleftrightarrow \\
\sum_{j \in S_{i}^{\prime}} \alpha_{i j} p_{j}^{\prime}-\sum_{j \in S_{i}} \alpha_{i j} p_{j}^{\prime}-a_{i}\left(S_{i}^{\prime}\right)+a_{i}\left(S_{i}\right) \leq 0 \Longleftrightarrow \\
k_{i}\left(S_{i}^{\prime}, a_{i}, p^{\prime}\right)-k_{i}\left(S_{i}, a_{i}, p^{\prime}\right) \leq 0 .
\end{gathered}
$$

Proof of Proposition 3. In this case, the technology function $A_{i}$ maps a set $S_{i}$ to a vector $\left(A_{i j}\right)_{j \in S_{i}}$. Write the CES cost function for firm $i$ as

$$
K_{i}\left(S_{i}, A_{i}, P\right)=\left(\left(1-\sum_{j \in S_{i}} \alpha_{i j}\right)^{\sigma}+\sum_{j \in S_{i}} \alpha_{i j}^{\sigma}\left(\frac{P_{j}}{A_{i j}}\right)^{1-\sigma}\right)^{\frac{1}{1-\sigma}} .
$$

Since the single-crossing condition is preserved by monotone transformations, it suffices to consider a monotone transformation of $K_{i}$. We split the analysis into two cases:

Case 1: $\sigma<1$

In this case, we can raise the cost function to the power $1-\sigma$ to obtain $\left(K_{i}\left(S_{i}, A_{i}, P\right)\right)^{1-\sigma}=$ $\left(1-\sum_{j \in S_{i}} \alpha_{i j}\right)^{\sigma}+\sum_{j \in S_{i}} \alpha_{i j}^{\sigma}\left(\frac{P_{j}}{A_{i j}}\right)^{1-\sigma}$. Since $1-\sigma>0$, minimizing $K_{i}$ is equivalent to minimizing $\left(K_{i}\left(S_{i}, A_{i}, P\right)\right)^{1-\sigma}$. We will show that $\left(K_{i}\left(S_{i}, A_{i}, P\right)\right)^{1-\sigma}$ satisfies the single-crossing condition. Let 
$S_{i} \subset S_{i}^{\prime}$ and $P^{\prime} \leq P$. We can derive the chain of implications

$$
\begin{gathered}
\left(K_{i}\left(S_{i}^{\prime}, A_{i}\left(S_{i}^{\prime}\right), P\right)\right)^{1-\sigma}-\left(K_{i}\left(S_{i}, A_{i}\left(S_{i}\right), P\right)\right)^{1-\sigma} \leq 0 \Longrightarrow \\
\left(\left(1-\sum_{j \in S_{i}^{\prime}} \alpha_{i j}\right)^{\sigma}-\left(1-\sum_{j \in S_{i}} \alpha_{i j}\right)^{\sigma}\right)+\sum_{j \in S_{i}^{\prime}-S_{i}} \alpha_{i j}^{\sigma}\left(\frac{P_{j}}{A_{i j}}\right)^{1-\sigma} \leq 0 \Longrightarrow \\
\left(\left(1-\sum_{j \in S_{i}^{\prime}} \alpha_{i j}\right)^{\sigma}-\left(1-\sum_{j \in S_{i}} \alpha_{i j}\right)^{\sigma}\right)+\sum_{j \in S_{i}^{\prime}-S_{i}} \alpha_{i j}^{\sigma}\left(\frac{P_{j}^{\prime}}{A_{i j}}\right)^{1-\sigma} \leq 0 \Longrightarrow \\
\left(K_{i}\left(S_{i}^{\prime}, A_{i}\left(S_{i}^{\prime}\right), P^{\prime}\right)\right)^{1-\sigma}-\left(K_{i}\left(S_{i}, A_{i}\left(S_{i}\right), P^{\prime}\right)\right)^{1-\sigma} \leq 0 .
\end{gathered}
$$

so the single-crossing condition is satisfied.

Case 2: $\sigma>1$

In this case, we can raise the cost function to the power $1-\sigma$ to obtain $\left(K_{i}\left(S_{i}, A_{i}, P\right)\right)^{1-\sigma}=$ $\left(1-\sum_{j \in S_{i}} \alpha_{i j}\right)^{\sigma}+\sum_{j \in S_{i}} \alpha_{i j}^{\sigma}\left(\frac{P_{j}}{A_{i j}}\right)^{1-\sigma}$. Since $1-\sigma<0$, minimizing $K_{i}$ is equivalent to maximizing $\left(K_{i}\left(S_{i}, A_{i}, P\right)\right)^{1-\sigma}$. We need to show that $\left(K_{i}\left(S_{i}, A_{i}, P\right)\right)^{1-\sigma}$ satisfies a reverse single-crossing condition. For all $S_{i} \subset S_{i}^{\prime}$ and $P^{\prime} \leq P\left(K_{i}\left(S_{i}^{\prime}, A_{i}\left(S_{i}^{\prime}\right), P\right)\right)^{1-\sigma}-\left(K_{i}\left(S_{i}, A_{i}\left(S_{i}\right), P\right)\right)^{1-\sigma} \geq 0 \Longrightarrow$ $\left(K_{i}\left(S_{i}^{\prime}, A_{i}\left(S_{i}^{\prime}\right), P^{\prime}\right)\right)^{1-\sigma}-\left(K_{i}\left(S_{i}, A_{i}\left(S_{i}^{\prime}\right), P^{\prime}\right)\right)^{1-\sigma} \geq 0$.

Let $S_{i} \subset S_{i}^{\prime}$ and $P^{\prime} \leq P$. Since $\left(\frac{P_{j}}{A_{i j}}\right)^{1-\sigma} \leq\left(\frac{P_{j}^{\prime}}{A_{i j}}\right)^{1-\sigma}$, we obtain the chain of implications

$$
\begin{gathered}
\left(K_{i}\left(S_{i}^{\prime}, A_{i}\left(S_{i}^{\prime}\right), P\right)\right)^{1-\sigma}-\left(K_{i}\left(S_{i}, A_{i}\left(S_{i}\right), P\right)\right)^{1-\sigma} \geq 0 \Longrightarrow \\
\left(\left(1-\sum_{j \in S_{i}^{\prime}} \alpha_{i j}\right)^{\sigma}-\left(1-\sum_{j \in S_{i}} \alpha_{i j}\right)^{\sigma}\right)+\sum_{j \in S_{i}^{\prime}-S_{i}} \alpha_{i j}^{\sigma}\left(\frac{P_{j}}{A_{i j}}\right)^{1-\sigma} \geq 0 \Longrightarrow \\
\left(\left(1-\sum_{j \in S_{i}^{\prime}} \alpha_{i j}\right)^{\sigma}-\left(1-\sum_{j \in S_{i}} \alpha_{i j}\right)^{\sigma}\right)+\sum_{j \in S_{i}^{\prime}-S_{i}} \alpha_{i j}^{\sigma}\left(\frac{P_{j}^{\prime}}{A_{i j}}\right)^{1-\sigma} \geq 0 \Longrightarrow \\
\left(K_{i}\left(S_{i}^{\prime}, A_{i}\left(S_{i}^{\prime}\right), P^{\prime}\right)\right)^{1-\sigma}-\left(K_{i}\left(S_{i}, A_{i}\left(S_{i}\right), P^{\prime}\right)\right)^{1-\sigma} \leq 0 .
\end{gathered}
$$

so the single-crossing condition is satisfied.

Proof of Theorem 6. Let $S_{i}^{O}(t)$ be the input combination available to industry $i$ at time $t$. Let $S_{i}^{*}(t)$ be the set that minimizes industry $i$ 's unit cost when it chooses between $S_{i}^{*}(t-1)$ and $S_{i}^{O}(t)$. Clearly,

$$
a_{i}\left(S_{i}^{*}(t)\right) \leq \max _{j \in\{1, \ldots, t\}} \max _{\tau \in\{1, \ldots, t\}} a_{j}\left(S_{j}^{O}(\tau)\right) .
$$

Therefore, denoting the equilibrium log productivity sequence by $a\left(S^{*}(t)\right)$, we have

$$
-\frac{\pi(t)}{t}=\frac{1}{t} \beta(t)^{\prime} \mathcal{L}(t) a\left(S^{*}(t)\right) \leq \frac{1}{t} \max _{j \in\{1, \ldots, t\}} \max _{\tau \in\{1, \ldots, t\}} a_{j}\left(S_{j}^{O}(\tau)\right) \beta(t)^{\prime} \mathcal{L}(t) 1(t),
$$

where $1(t)$ is a $t \times 1$ vector all of whose components are ones. Since $\beta(t)^{\prime} \mathcal{L}(t) 1(t)=\sum_{i, j=1}^{n} \beta_{j} \mathcal{L}_{i j}$ 
and $\sum_{j=1}^{\infty} \beta_{j}=1$, this implies

$$
\begin{aligned}
\limsup _{t \rightarrow \infty}\left(-\frac{\pi(t)}{t}\right) & =\limsup _{t \rightarrow \infty} \frac{1}{t} \max _{j \in\{1, \ldots, t\}} \max _{\tau \in\{1, \ldots, t\}} a_{j}\left(S_{j}^{O}(\tau)\right) \beta(t)^{\prime} \mathcal{L}(t) 1(t) \\
& \leq \limsup _{t \rightarrow \infty} \frac{1}{1-\theta} \frac{1}{t} \max _{j \in\{1, \ldots, t\}} \max _{\tau \in\{1, \ldots, t\}} a_{j}\left(S_{j}^{O}(\tau)\right) \\
& =\limsup _{t \rightarrow \infty} \frac{D}{1-\theta} \frac{\log _{2}\left(t^{2}\right)}{t}=0 \text { almost surely }
\end{aligned}
$$

where the last equality follows from Assumption 2.

Since $\liminf t \rightarrow \infty\left(-\frac{\pi(t)}{t}\right) \geq 0$ (as additional technology choices cannot increase prices), the previous argument establishes that $g^{*}=\lim _{t \rightarrow \infty}=\left(-\frac{\pi(t)}{t}\right)=0$.

Proof of Theorem 7. Let $\epsilon>0$ and $T(\epsilon)$ be such that for all $i \in \mathbb{N}, \sum_{j=T(\epsilon)}^{\infty} \alpha_{i j} \leq \epsilon$. Recall that $\alpha$ is the entire matrix of input-output elasticities, while $\alpha(S)$ is the observed matrix of inputoutput elasticities when the input-output network is given by $S$. Assumption 3 tells us that if $S_{i} \supset\{1, \ldots, T(\epsilon)\}$ for all $i$, we will have $\sum_{j=1}^{t} \alpha_{i j}(S) \geq \sum_{j=1}^{t} \alpha_{i j}-\epsilon$.

We next make use of the following lemma:

Lemma A2 Let $\alpha$ and $\beta$ be non-negative matrices $n \times n$ matrices. Let $A=(I-\alpha)^{-1}$ and $B=$ $(I-\beta)^{-1}$. If

- $\|\alpha\|_{\infty} \leq \theta,\|\beta\|_{\infty} \leq \theta$ for some $\theta<1$, and

- $\sum_{j=1}^{n} \beta_{i j} \geq\left(\sum_{j=1}^{n} \alpha_{i j}\right)-\epsilon$ for every row $i$, then $\sum_{j=1}^{n} B_{i j} \geq\left(\sum_{j=1}^{n} A_{i j}\right)-\frac{1}{(1-\theta)^{2}} \epsilon$ for every row $i$.

Proof of Lemma A2. Let $\alpha_{i j}^{\ell}$ be the $(i, j)$ element of the matrix $\alpha^{\ell}$. Since $A=\sum_{\ell=0}^{\infty} \alpha^{\ell}, B=$ $\sum_{\ell=0}^{\infty} \beta^{\ell}$ and $\sum_{\ell=1}^{\infty} \ell \theta^{\ell-1}=\frac{1}{(1-\theta)^{2}}$, it suffices to show that, for all $\ell \geq 0$ we have $\sum_{j=1}^{n} \beta_{i j}^{\ell} \geq$ $\left(\sum_{j=1}^{n} \alpha_{i j}^{\ell}\right)-\ell \theta^{\ell-1} \epsilon$. We proceed by induction. The base case $(\ell=1)$ is our assumption that $\sum_{j=1}^{n} \beta_{i j} \geq\left(\sum_{j=1}^{n} \alpha_{i j}\right)-\epsilon$. To prove the inductive case, assume we have shown the hypothesis for $\ell$, and we want to show it for $\ell+1$. Write

$$
\sum_{j} \beta_{i j}^{\ell+1}=\sum_{j} \sum_{k} \beta_{i k} \beta_{k j}^{\ell}=\sum_{k} \beta_{i k} \sum_{j} \beta_{k j}^{\ell} .
$$

By induction, this is greater than or equal to

$$
\sum_{k} \beta_{i k} \sum_{j} \alpha_{k j}^{\ell}-\sum_{k} \beta_{i k} \ell \theta^{\ell-1} \epsilon
$$

We now use the fact that $\sum_{k} \alpha_{i k}-\epsilon \leq \sum_{k} \beta_{i k} \leq \theta$ to infer that $\sum_{k} \beta_{i k} \sum_{j} \alpha_{k j}^{\ell}-\sum_{k} \beta_{i k} \ell \theta^{\ell-1} \epsilon$ is bounded below by

$$
\sum_{k} \alpha_{i k} \sum_{j} \alpha_{k j}^{\ell}-\epsilon \sum_{j} \alpha_{k j}^{\ell}-\theta \ell \theta^{\ell-1} \epsilon .
$$


The first term in the above expression is equal to $\sum_{j} \sum_{k} \alpha_{i k} \alpha_{k j}^{\ell}=\sum_{j} \alpha_{i j}^{\ell+1}$. The second term is bounded below by $-\epsilon\left\|\alpha^{\ell}\right\|_{\infty} \geq-\epsilon \theta^{\ell}$. We conclude that

$$
\sum_{j} \beta_{i j}^{\ell+1} \geq \sum_{j} \alpha_{i j}^{\ell+1}-(\ell+1) \theta^{\ell} \epsilon
$$

Adding up over all $\ell \in \mathbb{N}$, we obtain

$$
\sum_{j} B_{i j} \geq \sum_{j} A_{i j}-\frac{1}{(1-\theta)^{2}} \epsilon
$$

From this lemma, we can infer that for any $S \supset\{1, \ldots, T(\epsilon)\}, \sum_{j=1}^{t} \mathcal{L}_{i j}(S) \geq \sum_{j=1}^{t} \mathcal{L}_{i j}-\frac{1}{(1-\theta)^{2}} \epsilon$, which we will use in the proof that follows.

We first prove that $\liminf _{t \rightarrow \infty}-\frac{p_{i}^{*}(t)}{t \sum_{j=1}^{t} \mathcal{L}_{i j}} \geq D$. We will first show that this is the case even if industry $i$ chooses a suboptimal set of inputs corresponding to those with the highest levels of log productivity (rather than the cost-minimizing bundles), and then infer from this that it is also true for the equilibrium price sequence. Let us define $S_{i}^{0}(t)=\arg \max _{S_{i} \supset\{1, \ldots, T(\epsilon)\} \backslash\{i\}} a_{i}\left(S_{i}\right)$, $S^{0}(t)=\left\{S_{i}^{0}(t)\right\}_{i=1}^{t}$, and define $p_{i}^{0}(t)=-\sum_{j=1}^{t} \mathcal{L}_{i j}\left(S^{0}(t)\right) a_{j}\left(S_{j}^{0}(t)\right)$. The value $a_{i}\left(S_{i}^{0}(t)\right)$ is the maximum of $2^{t-1-T(\epsilon)}$ random variables. Then Assumption 2 implies that $\lim _{t \rightarrow \infty} \frac{a_{i}\left(S_{i}^{0}(t)\right)}{t-1-T(\epsilon)}=D$ almost surely. Since $T(\epsilon)$ is a constant independent of $t$, we have $\lim _{t \rightarrow \infty} \frac{a_{i}\left(S_{i}^{0}(t)\right)}{t}=D$ almost surely. Since a countable intersection of almost sure events happens almost surely, we also have $\lim _{t \rightarrow \infty} \min _{i} \frac{a_{i}\left(S_{i}^{0}(t)\right)}{t}=D$ almost surely. Furthermore, since $S_{i}^{0}(t) \supset\{1, \ldots, T(\epsilon)\} \backslash\{i\}$, we have $\sum_{j=1}^{t} \mathcal{L}_{i j}\left(S^{0}(t)\right) \geq \sum_{j=1}^{t} \mathcal{L}_{i j}-\frac{1}{(1-\theta)^{2}} \epsilon$. Plugging these bounds into the definition of $p_{i}^{0}$, we obtain

$$
\begin{aligned}
-p_{i}^{0}(t)=\sum_{j=1}^{t} \mathcal{L}_{i j}(S(t)) a_{j}\left(S_{j}^{0}(t)\right) & \geq \min _{k \leq t} a_{k}\left(S_{k}^{0}(t)\right) \sum_{j=1}^{t} \mathcal{L}_{i j}(S(t)) \\
& \geq \min _{k \leq t} a_{k}\left(S_{k}^{0}(t)\right)\left(\sum_{j=1}^{t} \mathcal{L}_{i j}-\frac{1}{(1-\theta)^{2}} \epsilon\right) .
\end{aligned}
$$

Dividing both sides by $t \sum_{j=1}^{t} \mathcal{L}_{i j}$, we obtain

$$
-\frac{p_{i}^{0}(t)}{t \sum_{j=1}^{t} \mathcal{L}_{i j}} \geq \frac{\min _{k \leq t} a_{k}\left(S_{k}^{0}(t)\right)}{t}-\epsilon \frac{\min _{k \leq t} a_{k}\left(S_{k}^{0}(t)\right)}{t(1-\theta)^{2} \sum_{j=1}^{t} \mathcal{L}_{i j}} .
$$

Using the fact that $\sum_{j=1}^{t} \mathcal{L}_{i j} \geq 1$, this inequality can be written as

$$
-\frac{p_{i}^{0}(t)}{t \sum_{j=1}^{t} \mathcal{L}_{i j}} \geq \frac{\min _{k \leq t} a_{k}\left(S_{k}^{0}(t)\right)}{t}-\epsilon \frac{\min _{k \leq t} a_{k}\left(S_{k}^{0}(t)\right)}{t(1-\theta)^{2}} .
$$

Taking lim inf on both sides, we obtain

$$
\liminf _{t \rightarrow \infty}-\frac{p_{i}^{0}(t)}{t \sum_{j=1}^{t} \mathcal{L}_{i j}} \geq D-\epsilon D \frac{1}{(1-\theta)^{2}} .
$$


Since $\epsilon$ is arbitrarily small, we conclude that

$$
\liminf _{t \rightarrow \infty}-\frac{p_{i}^{0}(t)}{t \sum_{j=1}^{t} \mathcal{L}_{i j}} \geq D
$$

With the same arguments as in the proof of Theorem 1, we also have that the function $\kappa(p)=\left(\min _{S_{1}} k_{1}\left(a_{1}\left(S_{1}\right), S_{1}, p\right), \ldots, \min _{S_{n}} k_{n}\left(a_{n}\left(S_{n}\right), S_{n}, p\right)\right)$ has a smallest fixed point which gives the equilibrium log price vector $p^{*}(t)$. Starting from $p^{0}(t)$, we can define a decreasing sequence $p^{\tau}(t)=\kappa\left(p^{\tau-1}(t)\right)$ which converges to a fixed point $p(t)$ of $\kappa$. Since the equilibrium log price vector is the lowest fixed point of $\kappa$, we have that $p^{*}(t) \leq p(t) \leq p^{0}(t)$. Dividing by $t \sum_{j=1}^{t} \mathcal{L}_{i j}$ and taking liminf on both sides, we conclude

$$
\liminf _{t \rightarrow \infty}-\frac{p_{i}^{*}(t)}{t \sum_{j=1}^{t} \mathcal{L}_{i j}} \geq D
$$

To prove that $\limsup _{t \rightarrow \infty} \frac{-p_{i}^{*}(t)}{t \sum_{j=1}^{t} \mathcal{L}_{i j}} \leq D$, let us write $-p_{i}^{*}(t)=\sum_{j=1}^{t} \mathcal{L}_{i j}(S(t)) a_{j}\left(S_{j}(t)\right) \leq$ $\max _{k \leq t} a_{k}\left(S_{k}(t)\right) \sum_{j=1}^{t} \mathcal{L}_{i j}$. The value of $\max _{k \leq t} a_{k}\left(S_{k}(t)\right)$ can be upper bounded by $\max _{k \leq t} \max _{S_{k}^{\prime}} a_{k}\left(S_{k}^{\prime}\right)$, which is the maximum of $t \times 2^{t-1}$ i.i.d. draws from the distribution $\Phi$. From Assumption 2, we have $\lim \sup _{t \rightarrow \infty} \frac{\max _{k \leq t} \max _{S_{k}^{\prime}} a_{k}\left(S_{k}^{\prime}\right)}{\log _{2}(t)+t-1} \leq D$. Since $\log _{2}(t)-1=o(t)$, we have that $\limsup _{t \rightarrow \infty} \frac{\max _{k \leq t} \max _{S_{k}^{\prime}} a_{k}\left(S_{k}^{\prime}\right)}{t} \leq D$. Dividing $-p_{i}^{*}(t)$ by $t \sum_{j=1}^{t} \mathcal{L}_{i j}$ and taking limsup on both sides, we obtain

$$
\limsup _{t \rightarrow \infty}-\frac{p_{i}(t)}{t \sum_{j=1}^{t} \mathcal{L}_{i j}} \leq \limsup _{t \rightarrow \infty} \frac{\max _{k \leq t} \max _{S_{k}^{\prime}} a_{k}\left(S_{k}^{\prime}\right)}{t} \leq D \text { almost surely. }
$$

Combining this with (A1), we can thus conclude that

$$
\lim _{t \rightarrow \infty}-\frac{p_{i}^{*}(t)}{t \sum_{j=1}^{t} \mathcal{L}_{i j}}=D \text { almost surely, }
$$

and thus

$$
g^{*}=\lim _{t \rightarrow \infty}\left(-\frac{\pi(t)}{t}\right)=D \sum_{i, j=1}^{\infty} \beta_{i} \mathcal{L}_{i j} \text { almost surely }
$$

\section{Proof of Corollary 3.}

Let $\widetilde{\alpha}_{i j}=\left\{\begin{array}{l}\alpha_{i j} \text { if }(i, j) \notin R \\ 0 \text { otherwise }\end{array}\right.$ be the exogenous input-output matrix after the direct restrictions in $R$ are applied. Let $\epsilon>0$ and $T(\epsilon)$ be such that for all $i \in \mathbb{N}, \sum_{j=T(\epsilon)}^{\infty} \widetilde{\alpha}_{i j} \leq \epsilon$. Let $R_{i}=\{j:(i, j) \epsilon$ $R\}$. From Assumption 3, when $S_{i} \supset\{1, \ldots, T(\epsilon)\} \backslash R_{i}$ for all $i$, we have $\sum_{j=1}^{t} \widetilde{\alpha}_{i j}(S) \geq \sum_{j=1}^{t} \widetilde{\alpha}_{i j}-\epsilon$.

From Lemma A2, when $S_{i} \supset\{1, \ldots, T(\epsilon)\} \backslash R_{i}$ for all $i$, we have $\sum_{j=1}^{t} \widetilde{\mathcal{L}}_{i j}(S) \geq \sum_{j=1}^{t} \mathcal{L}_{i j}-\frac{1}{(1-\theta)^{2}} \epsilon$. We will use these facts in the proof that follows. 
We first prove that $\liminf _{t \rightarrow \infty}-\frac{p_{i}^{*}(t)}{t \sum_{j=1}^{t} \widetilde{\mathcal{L}}_{i j}} \geq(1-\rho) D$. We first show that this is the case even if industry $i$ chooses a suboptimal set of inputs corresponding to those with the highest levels of log productivity (rather than the cost-minimizing input combination), which implies that the same is a fortiori true for the equilibrium price sequence. Let us define $S_{i}^{0}(t)=\arg \max _{S_{i} \supset\{1, \ldots, T(\epsilon)\} \backslash\left(R_{i} \cup\{i\}\right)} a_{i}\left(S_{i}\right), \quad S^{0}(t)=\left\{S_{i}^{0}(t)\right\}_{i=1}^{t}$, and let $p_{i}^{0}(t)=$ $-\sum_{j=1}^{t} \widetilde{\mathcal{L}}_{i j}\left(S^{0}(t)\right)\left(a_{j}\left(S_{j}^{0}(t)\right)+\log \left(1+\tau_{j}\right)\right)$. The value $a_{i}\left(S_{i}^{0}(t)\right)$ is the maximum of at least $2^{t-\left|R_{i}\right|-1-T(\epsilon)}$ random variables. Then Assumption 2 implies that $\lim _{t \rightarrow \infty} \frac{a_{i}\left(S_{i}^{0}(t)\right)}{t-\left|R_{i}\right|-1-T(\epsilon)} \geq D$ almost surely. Since $T(\epsilon)$ is a constant independent of $t$, we have $\lim _{t \rightarrow \infty} \frac{a_{i}\left(S_{i}^{0}(t)\right)}{t-\left|R_{i}\right|} \geq D$ almost surely. Since each $j$ is in $R_{i}$ randomly with probability $\rho$, the $\operatorname{limit}_{t \rightarrow \infty} \frac{\left|R_{i}\right|}{t}=\rho$ holds almost surely as well, and we can write

$$
\lim _{t \rightarrow \infty} \frac{a_{i}\left(S_{i}^{0}(t)\right)}{t(1-\rho)} \geq D \text { almost surely. }
$$

Since a countable intersection of almost sure events happens almost surely, we also have $\lim _{t \rightarrow \infty} \min _{i} \frac{a_{i}\left(S_{i}^{0}(t)\right)}{t(1-\rho)} \geq D$ almost surely. Furthermore, since $S_{i}^{0}(t) \supset\{1, \ldots, T(\epsilon)\} \backslash\left(R_{i} \cup\{i\}\right)$, we have $\sum_{j=1}^{t} \widetilde{\mathcal{L}}_{i j}\left(S^{0}(t)\right) \geq \sum_{j=1}^{t} \widetilde{\mathcal{L}}_{i j}-\frac{1}{(1-\theta)^{2}} \epsilon$. Substituting these bounds into the formula for $p_{i}^{0}$, we obtain

$$
\begin{aligned}
-p_{i}^{0}(t) & =\sum_{j=1}^{t} \widetilde{\mathcal{L}}_{i j}(S(t))\left(a_{j}\left(S_{j}^{0}(t)\right)+\log \left(1+\tau_{j}\right)\right) \geq \min _{k \leq t} a_{k}\left(S_{k}^{0}(t)\right) \sum_{j=1}^{t} \widetilde{\mathcal{L}}_{i j}(S(t))+\sum_{j=1}^{t} \widetilde{\mathcal{L}}_{i j}(S(t)) \log \left(1+\tau_{j}\right) \\
& \geq \min _{k \leq t} a_{k}\left(S_{k}^{0}(t)\right)\left(\sum_{j=1}^{t} \widetilde{\mathcal{L}}_{i j}-\frac{1}{(1-\theta)^{2}} \epsilon\right)+\sum_{j=1}^{t} \widetilde{\mathcal{L}}_{i j}(S(t)) \log \left(1+\tau_{j}\right) .
\end{aligned}
$$

Dividing both sides by $t \sum_{j=1}^{t} \widetilde{\mathcal{L}}_{i j}$, we obtain

$$
-\frac{p_{i}^{0}(t)}{t \sum_{j=1}^{t} \widetilde{\mathcal{L}}_{i j}} \geq \frac{\min _{k \leq t} a_{k}\left(S_{k}^{0}(t)\right)}{t}-\epsilon \frac{\min _{k \leq t} a_{k}\left(S_{k}^{0}(t)\right)}{t(1-\theta)^{2} \sum_{j=1}^{t} \widetilde{\mathcal{L}}_{i j}}+\frac{\sum_{j=1}^{t} \widetilde{\mathcal{L}}_{i j}(S(t)) \log \left(1+\tau_{j}\right)}{t \sum_{j=1}^{t} \widetilde{\mathcal{L}}_{i j}(S(t))} .
$$

Using the fact that $\sum_{j=1}^{t} \widetilde{\mathcal{L}}_{i j} \geq 1$, this inequality can be written as

$$
-\frac{p_{i}^{0}(t)}{t \sum_{j=1}^{t} \widetilde{\mathcal{L}}_{i j}} \geq \frac{\min _{k \leq t} a_{k}\left(S_{k}^{0}(t)\right)}{t}-\epsilon \frac{\min _{k \leq t} a_{k}\left(S_{k}^{0}(t)\right)}{t(1-\theta)^{2}}+\frac{\sum_{j=1}^{t} \widetilde{\mathcal{L}}_{i j}(S(t)) \log \left(1+\tau_{j}\right)}{t \sum_{j=1}^{t} \widetilde{\mathcal{L}}_{i j}(S(t))} .
$$

Taking $\lim \inf _{t \rightarrow \infty}$ on both sides, the term $\frac{\sum_{j=1}^{t} \widetilde{\mathcal{L}}_{i j}(S(t)) \log \left(1+\tau_{j}\right)}{t \sum_{j=1}^{t} \widetilde{\mathcal{L}}_{i j}(S(t))}$ vanishes because $\sum_{j=1}^{t} \widetilde{\mathcal{L}}_{i j}(S(t)) \leq$ $\frac{1}{1-\theta}$ and because $\tau_{j}$ does not vary with $t$. This yields the inequality

$$
\liminf _{t \rightarrow \infty}-\frac{p_{i}^{0}(t)}{t \sum_{j=1}^{t} \widetilde{\mathcal{L}}_{i j}} \geq(1-\rho) D-\epsilon(1-\rho) D \frac{1}{(1-\theta)^{2}}
$$

Since $\epsilon$ is arbitrarily small, we conclude that

$$
\liminf _{t \rightarrow \infty}-\frac{p_{i}^{0}(t)}{t \sum_{j=1}^{t} \widetilde{\mathcal{L}}_{i j}} \geq(1-\rho) D
$$


With the same arguments as in the proof of Theorem 1, we also have that the function $\kappa(p)=\left(\min _{S_{1}} k_{1}\left(a_{1}\left(S_{1}\right), S_{1}, p\right), \ldots, \min _{S_{n}} k_{n}\left(a_{n}\left(S_{n}\right), S_{n}, p\right)\right)$ has a smallest fixed point which gives the equilibrium log price vector $p^{*}(t)$. Starting from $p^{0}(t)$, we can define a decreasing sequence $p^{\tau}(t)=\kappa\left(p^{\tau-1}(t)\right)$ which converges to a fixed point $p(t)$ of $\kappa$. Since the equilibrium log price vector is the lowest fixed point of $\kappa$, we have that $p^{*}(t) \leq p(t) \leq p^{0}(t)$. Dividing by $t \sum_{j=1}^{t} \widetilde{\mathcal{L}}_{i j}$ and taking liminf on both sides, we conclude

$$
\liminf _{t \rightarrow \infty}-\frac{p_{i}^{*}(t)}{t \sum_{j=1}^{t} \widetilde{\mathcal{L}}_{i j}} \geq(1-\rho) D
$$

To prove that $\lim \sup _{t \rightarrow \infty} \frac{-p_{i}^{*}(t)}{t \sum_{j=1}^{t} \widetilde{\widetilde{L}}_{i j}} \leq(1-\rho) D$, let us write $-p_{i}^{*}(t)=\sum_{j=1}^{t} \widetilde{\mathcal{L}}_{i j}(S(t))\left(a_{j}\left(S_{j}(t)\right)+\right.$ $\left.\log \left(1+\tau_{j}\right)\right) \leq \max _{k \leq t} a_{k}\left(S_{k}(t)\right) \sum_{j=1}^{t} \widetilde{\mathcal{L}}_{i j}+\sum_{j=1}^{t} \widetilde{\mathcal{L}}_{i j} \log \left(1+\tau_{j}\right)$. The value of $\max _{k \leq t} a_{k}\left(S_{k}(t)\right)$ can be upper bounded by $\max _{k \leq t} \max _{\left\{S_{k}^{\prime}: S_{k}^{\prime} \cap R_{k}=\varnothing\right\}} a_{k}\left(S_{k}^{\prime}\right)$, which is the maximum of at least $t \times 2^{t-1-\left|R_{k}\right|}$ i.i.d. draws from the distribution $\Phi$. From Assumption 2, we have $\limsup _{t \rightarrow \infty} \frac{\max _{k \leq t} \max _{\left\{S_{k}^{\prime}: S_{k}^{\prime} \cap R_{k}=\varnothing\right\}} a_{k}\left(S_{k}^{\prime}\right)}{\log 2(t)+t-1-\left|R_{k}\right|} \leq D$. Since $\log _{2}(t)-1=o(t)$, we have that

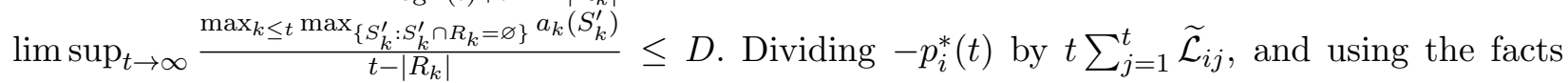
that $\lim _{t \rightarrow \infty} \frac{\left|R_{k}\right|}{t}=\rho, \lim _{t \rightarrow \infty} \frac{1+\tau_{j}}{t}=0$ almost surely, we can take lim sup on both sides to obtain

$$
\limsup _{t \rightarrow \infty}-\frac{p_{i}(t)}{t \sum_{j=1}^{t} \widetilde{\mathcal{L}}_{i j}} \leq \limsup _{t \rightarrow \infty} \frac{\max _{k \leq t} \max _{\left\{S_{k}^{\prime}: S_{k}^{\prime} \cap R_{k}=\varnothing\right\}} a_{k}\left(S_{k}^{\prime}\right)}{t} \leq(1-\rho) D \text { almost surely. }
$$

Combining this with (A3), we can thus conclude that

$$
\lim _{t \rightarrow \infty}-\frac{p_{i}^{*}(t)}{t \sum_{j=1}^{t} \widetilde{\mathcal{L}}_{i j}}=(1-\rho) D \text { almost surely, }
$$

and thus

$$
g^{*}=\lim _{t \rightarrow \infty}\left(-\frac{\sum_{i=1}^{t} \beta_{i}\left(p_{i}(t)+\log \left(1+\tau_{i}\right)\right)}{t}\right)=(1-\rho) D \sum_{i, j=1}^{\infty} \beta_{i} \widetilde{\mathcal{L}}_{i j} \text { almost surely }
$$

where the last equation uses the fact that log GDP now depends on the vector of marginal distortions $\tau$, but our growth rate is unaffected by them since $\lim _{t \rightarrow \infty} \frac{\left.\log \left(1+\tau_{i}\right)\right)}{t}=0$.

Proof of Lemma 3. The log unit cost of adopting set $S_{i}$ is $k_{i}\left(S_{i}, a_{i}\left(S_{i}\right), p\right)=\sum_{j \in S_{i}} \alpha_{i j} p_{j}-$ $a_{i}\left(S_{i}\right)$ where $a_{i}\left(S_{i}\right)$ is distributed according to a Gumbel distribution with variance parameter $\sigma$. Choosing $S_{i}$ to minimize $k_{i}\left(S_{i}, a_{i}\left(S_{i}\right), p\right)$ is equivalent to choosing $S_{i}$ to maximize $-k_{i}\left(S_{i}, a_{i}\left(S_{i}\right), p\right)=$ $-\sum_{j \in S_{i}} \alpha_{i j} p_{j}+a_{i}\left(S_{i}\right)$. Part 1 then follows from the same derivation as that of Lemma 1 in McFadden (1973). 
Part 2 follows because

$$
\begin{aligned}
\operatorname{Pr}\left(j \in S_{i} \mid P\right) & =\frac{\sum_{S_{i}: j \in S_{i}} \prod_{j^{\prime} \in S_{i}} P_{j^{\prime}}^{-\frac{\alpha_{i j^{\prime}}}{\sigma}}}{\sum_{S_{i}} \prod_{j^{\prime} \in S_{i}} P_{j^{\prime}}^{-\frac{\alpha_{i j^{\prime}}}{\sigma}}}=\frac{\sum_{S_{i}: j \in S_{i}} \prod_{j^{\prime} \in S_{i}} P_{j^{\prime}}^{-\frac{\alpha_{i j^{\prime}}}{\sigma}}}{\sum_{S_{i}: j \in S_{i}} \prod_{j^{\prime} \in S_{i}} P_{j^{\prime}}^{-\frac{\alpha_{i j^{\prime}}}{\sigma}}+\sum_{S_{i}: j \notin S_{i}} \prod_{j^{\prime} \in S_{i}} P_{j^{\prime}}^{-\frac{\alpha_{i j^{\prime}}}{\sigma}}} \\
& =\frac{P_{j}^{-\frac{\alpha_{i j}}{\sigma}} \sum_{S_{i}: j \notin S_{i}} \prod_{j^{\prime} \in S_{i}} P_{j^{\prime}}^{-\frac{\alpha_{i j^{\prime}}}{\sigma}}}{P_{j}^{-\frac{\alpha_{i j}}{\sigma}} \sum_{S_{i}: j \notin S_{i}} \prod_{j^{\prime} \in S_{i}} P_{j^{\prime}}^{-\frac{\alpha_{i j^{\prime}}}{\sigma}}+\sum_{S_{i}: j \notin S_{i}} \prod_{j^{\prime} \in S_{i}} P_{j^{\prime}}^{-\frac{\alpha_{i j^{\prime}}}{\sigma}}}=\frac{P_{j}^{-\frac{\alpha_{i j}}{\sigma}}}{1+P_{j}^{-\frac{\alpha_{i j}}{\sigma}}} .
\end{aligned}
$$

Proof of Theorem 8. Part 1. Since $\sum_{j=1}^{n} \alpha_{i j} \leq 1$ for every $i$ from Assumption $3^{\prime}, \mathcal{I}_{i}(n)=$ $\frac{1}{n} \sum_{j=1}^{n} \alpha_{i j}(S(n)) \leq \frac{1}{n}$. Thus, for every $\epsilon>0$, we have $\|\mathcal{I}(n)\|_{\infty} \leq \frac{1}{n}$. This implies that $\mathcal{I}(n)$ uniformly converges to 0 .

Part 2. We can write $\mathcal{O}_{j}(n)=\frac{1}{n} \sum_{i=1}^{n} \alpha_{i j} I(i, j, n)$, where $I(i, j, n)$ is an indicator function that is equal to 1 if $j \in S_{i}(n)$ and 0 otherwise. Since $I(i, j, n) \leq 1$,

$$
\mathcal{O}_{j}(n) \leq \frac{1}{n} \sum_{i=1}^{n} \alpha_{i j}=\alpha_{j}
$$

This implies that $\lim \sup _{n \rightarrow \infty} \mathcal{O}_{j}(n)=\overline{\mathcal{O}}_{j} \leq \alpha_{j}$ for all $j$.

Part 3. Let $P(n)$ be the price vector in economy $\mathcal{E}(n)$, and let $\mathcal{O}_{j}(n) \mid P(n)$ be the outdegree of $j$ conditional on prices. From Lemma 3, the decisions of any two industries $i, i^{\prime}$ on whether or not to choose $j$ as a supplier are independent given prices. Thus, the sequence of random variables $\{I(i, j, n) \mid P(n)\}_{i=1}^{n}$ is a sequence of independent Bernoulli random variables with $\operatorname{Pr}(I(i, j, n) \mid P(n))=\frac{P_{j}^{-\alpha_{i j}}}{1+P_{j}^{-\alpha_{i j}}}$. The expected outdegree of firm $j$ given a fixed price vector $P(n)$ is $\mathbb{E}\left[\mathcal{O}_{j}(n) \mid P(n)\right]=\frac{1}{n} \sum_{i=1}^{n} \alpha_{i j} \frac{P_{j}^{-\alpha_{i j}}}{1+P_{j}^{-\alpha_{i j}}}$. If $P_{j}(n) \leq 1$ for every $j \in \mathbb{N}$, then we have $\frac{P_{j}^{-\alpha_{i j}}}{1+P_{j}^{-\alpha_{i j}}} \geq \frac{1}{2}$, and $\mathbb{E}\left[\mathcal{O}_{j}(n) \mid P(n)\right] \geq \frac{1}{n} \sum_{i=1}^{n} \frac{\alpha_{i j}}{2}=\frac{1}{2} \alpha_{j}$.

Recall that if $X_{1}, \ldots, X_{n}$ are indpendent random variables in the interval $[0,1]$, we have the following Chernoff bound

$$
\operatorname{Pr}\left(\left|\frac{1}{n} \sum_{i=1}^{n} X_{i}-\frac{1}{n} \sum_{i=1}^{n} \mathbb{E}\left[X_{i}\right]\right| \geq \epsilon\right) \leq 2 e^{-2 n \epsilon^{2}} .
$$

Using this Chernoff bound and the conditional independence of each $I(i, j, n) \mid P(n)$, we get that for any $\epsilon>0$, we have $\operatorname{Pr}\left(\left|\mathcal{O}_{j}(n)-\mathbb{E}\left[\mathcal{O}_{j}(n) \mid P(n)\right]\right| \geq \epsilon\right) \leq 2 e^{-2 n \epsilon^{2}}$. Using the first Borel-Cantelli Lemma (Lemma B2) and the fact that $\sum_{n=1}^{\infty} 2 e^{-2 n \epsilon^{2}}<\infty$, we conclude that $\limsup _{n \rightarrow \infty} \mid \mathcal{O}_{j}(n)-$ $\mathbb{E}\left[\mathcal{O}_{j}(n) \mid P(n)\right] \mid \leq 0$ almost surely. Using the reverse triangle inequality and the fact that $\mathcal{O}_{j}(n) \geq 0$, this becomes

$$
\lim \sup _{n \rightarrow \infty} \mathbb{E}\left[\mathcal{O}_{j}(n) \mid P(n)\right] \leq \lim \inf _{n \rightarrow \infty} \mathcal{O}_{j}(n)=\underline{\mathcal{O}_{j}} \text { almost surely }
$$


Finally, recall from the proof of Theorem 7 that $\lim _{\sup _{n \rightarrow \infty}} \max _{j \leq n} p_{j}(n) \leq 0$ almost surely, and thus $\lim \sup _{n \rightarrow \infty} \max _{j \leq n} P_{j}(n) \leq 1$ almost surely (this result still holds, since the assumptions imposed here are stronger versions of those in Theorem 7). Therefore, $\lim \inf _{n \rightarrow \infty} \min _{j \leq n} \frac{P_{j}^{-\alpha_{i j}}}{1+P_{j}^{-\alpha_{i j}} \geq}$ $\frac{1}{2}$ almost surely, and consequently,

$$
\lim \sup _{n \rightarrow \infty} \frac{1}{2} \alpha_{j} \leq \lim \sup _{n \rightarrow \infty} \mathbb{E}\left[\mathcal{O}_{j}(n) \mid P(n)\right] \leq \underline{\mathcal{O}_{j}}
$$

holds almost surely for all $j \in \mathbb{N}$.

Finally, note that if $\overline{\mathcal{O}}$ were a degenerate distribution, then either $\overline{\mathcal{O}_{j}}=0$ for all $j$ or $\overline{\mathcal{O}_{j}}=\rho>0$ for all $j$. In the former case, we would have $\sum_{j=1}^{\infty} \overline{\mathcal{O}_{j}}=0$, which cannot be the case because $\overline{\mathcal{O}}>\frac{\alpha}{2}$ and $\sum_{j=1}^{\infty} \frac{\alpha_{j}}{2}>0$. In the latter case, we would have $\sum_{j=1}^{\infty} \overline{\mathcal{O}_{j}}=\infty$, which cannot be the case because $\overline{\mathcal{O}} \leq \alpha$ and $\sum_{j=1}^{\infty} \alpha_{j} \leq 1$. The argument that $\underline{\mathcal{O}}$ cannot be a degenerate distribution is analogous to the argument for $\overline{\mathcal{O}}$.

Proof of Theorem 9. Under logarithmic preferences as specified in Assumption 1', we have $\beta_{i}=P_{i} C_{i}$. Then the parameter vector $\beta$ is identified from the consumption expenditure shares $P_{1} C_{1}, \ldots, P_{n} C_{n}$. In particular, let $\widehat{\beta}(\mathcal{E}(n))=\left(P_{i} C_{i}\right)_{i=1}^{n}$, then $\operatorname{plim}_{n \rightarrow \infty} \widehat{\beta}(\mathcal{E}(n))=\beta$.

For $\sigma$, recall that the price index of the economy is given by $\pi(n)=\sum_{i=1}^{n} \beta_{i} \log P_{i}(n)$ and that $-\operatorname{plim}_{n \rightarrow \infty} \frac{\pi(n)}{n}=D \sum_{i, j=1}^{\infty} \beta_{i} \mathcal{L}_{i j}$. Under Assumption $2^{\prime}$, we have $D=\sigma \log 2$. Thus, we can set $\widehat{\sigma}(\mathcal{E}(n))=-\frac{\pi(n)}{n \log 2 \sum_{i, j=1}^{\infty} \beta_{i} \mathcal{L}_{i j}}$. From Theorem 7, plim $\lim _{n \rightarrow \infty} \widehat{\sigma}(\mathcal{E}(n))=\sigma$.

For $\alpha$, let $P(n)$ and $S(n)$ be the price vector and equilibrium network in economy $\mathcal{E}(n)$. Then consider the estimator $\widehat{\alpha}_{j}(\mathcal{E}(n))=\arg \min _{z} \frac{1}{n} \sum_{i=1}^{n}\left(\alpha_{i j}(S(n))-z P_{j}(n) \frac{P_{j}(n)^{-\frac{z}{\sigma}}}{1+P_{j}(n)^{-\frac{z}{\sigma}}}\right)^{2}$, where $\alpha_{i j}(S(n))$ is the $i j$-th and feel the observed input-output network $S(n)$.

Let $\Phi\left(z ; P_{j}(n)\right)=z \frac{P_{j}(n)^{-\frac{z}{\sigma}}}{1+P_{j}(n)^{-\frac{z}{\sigma}}}$. The derivative of this function with respect to $z$ is $\frac{\partial \Phi}{\partial z}=$ $\frac{1+P_{j}(n)^{\frac{z}{\sigma}}-P_{j}(n)^{\frac{z}{\sigma}} \log \left(P_{j}(n)^{\frac{z}{\sigma}}\right)}{\left(1+P_{j}(n)^{\frac{z}{\sigma}}\right)^{2} \sigma}$. For $P_{j}(n)<1, \Phi\left(z ; P_{j}(n)\right)$ is increasing in $z$. Furthermore, there exists a constant $K$ independent of $n$ such that $\frac{\partial \Phi}{\partial z} \leq K$ for all $P_{j}(n) \leq 1 .^{29}$ From Theorem 7 , we know that $\lim _{n \rightarrow \infty} P_{j}(n)<1$ almost surely. In the remainder of the proof, we will omit the qualifier "almost surely" for notational convenience and simply state that $P_{j}(n)<1$ and the derivative $\frac{\partial \Phi}{\partial z}$ is bounded.

\footnotetext{
${ }^{29}$ The term $\frac{1+P_{j}(n)^{\frac{z}{\sigma}}}{\left(1+P_{j}(n)^{\frac{z}{\sigma}}\right)^{2} \sigma}$ is clearly bounded by 1 . The term $\frac{-P_{j}(n)^{\frac{z}{\sigma}} \log \left(P_{j}(n)^{\frac{z}{\sigma}}\right)}{\left(1+P_{j}(n)^{\frac{z}{\sigma}}\right)^{2} \sigma}$ is bounded since the function $y^{z} \log y^{z}$ is bounded for every $y, z \in[0,1] \times[0,1]$ since $\lim _{y \rightarrow 0} y^{z} \log y^{z}=0$ for any fixed $z$.
} 
Since $\Phi\left(z ; P_{j}(n)\right)$ is increasing in $z$, we can write

$$
\begin{aligned}
\widehat{\alpha}_{j} & =\arg \min _{z} \frac{1}{n} \sum_{i=1}^{n}\left(\alpha_{i j}(S(n))-\Phi\left(z ; P_{j}(n)\right)\right)^{2} \\
& =\Phi^{-1}\left(\arg \min _{y} \frac{1}{n} \sum_{i=1}^{n}\left(\alpha_{i j}(S(n))-y\right)^{2}\right) \\
& =\Phi^{-1}\left(\frac{1}{n} \sum_{i=1}^{n} \alpha_{i j}(S(n))\right),
\end{aligned}
$$

where the first equality follows from the fact that $\Phi\left(z ; P_{j}(n)\right)$ is increasing in $z$ and the second equality follows from the fact that the the average value $\frac{1}{n} \sum_{i=1}^{n} \alpha_{i j}(S(n))$ minimizes the sum of square $\frac{1}{n} \sum_{i=1}^{n}\left(\alpha_{i j}(S(n))-y\right)^{2}$.

Note that each $\alpha_{i j}(S(n))$ is a random variable equal to an exogenous value $\alpha_{i j}$ with probability $\frac{P_{j}(n)^{-\frac{\alpha_{i j}}{\sigma}}}{1+P_{j}(n)^{-\frac{\alpha_{i j}}{\sigma}}}$ and equal to zero otherwise. These random variables are independent conditional on prices. From Assumption $3^{\prime}, \frac{1}{n} \sum_{i=1}^{n} \alpha_{i j}(S(n))$ converges to a constant as $n \rightarrow \infty$. Moreover, since the expectation of $\alpha_{i j}(S(n))$ is $\Phi\left(\alpha_{i j} ; P_{j}(n)\right)$, this limit is equal to $\lim _{n \rightarrow \infty} \frac{1}{n} \sum_{i=1}^{n} \Phi\left(\alpha_{i j} ; P_{j}(n)\right)$, which therefore also converges to a finite limit.

From the continuity of $\Phi^{-1}$, we have $\lim _{n \rightarrow \infty} \widehat{\alpha}_{j}=\Phi^{-1}\left(\lim _{n \rightarrow \infty} \frac{1}{n} \sum_{i=1}^{n} \Phi\left(\alpha_{i j} ; P_{j}(n)\right) ; P_{j}(n)\right)$. Letting $\alpha_{i j}=\alpha_{j}+\epsilon_{i j}$ and taking a Taylor expansion of $\Phi\left(\cdot ; P_{j}(n)\right)$ around $\alpha_{j}$, we obtain

$$
\Phi\left(\alpha_{i j} ; P_{j}(n)\right)=\Phi\left(\alpha_{j} ; P_{j}(n)\right)+\frac{\partial \Phi}{\partial z}\left(z_{i j} ; P_{j}(n)\right) \epsilon_{i j}
$$

for $\epsilon_{i j}=\alpha_{i j}-\alpha_{j}$ and some $z_{i j}$ between $\alpha_{i j}$ and $\alpha_{j}$. Substituting for this expression, we have

$$
\lim _{n \rightarrow \infty} \frac{1}{n} \sum_{i=1}^{n} \Phi\left(\alpha_{i j} ; P_{j}(n)\right)=\Phi\left(\alpha_{j} ; P_{j}(n)\right)+\lim _{n \rightarrow \infty} \frac{1}{n} \sum_{i=1}^{n} \frac{\partial \Phi}{\partial z}\left(z_{i j} ; P_{j}(n)\right) \epsilon_{i j} .
$$

Since $\frac{\partial \Phi}{\partial z}$ is a non-negative and bounded function and $\frac{1}{n} \sum_{i=1}^{n} \epsilon_{i j}=0$, $\lim _{n \rightarrow \infty} \frac{1}{n} \sum_{i=1}^{n} \frac{\partial \Phi}{\partial z}\left(z_{i j} ; P_{j}(n)\right) \epsilon_{i j}=0$. Therefore,

$$
\lim _{n \rightarrow \infty} \frac{1}{n} \sum_{i=1}^{n} \Phi\left(\alpha_{i j} ; P_{j}(n)\right)=\Phi\left(\alpha_{j}\right)
$$

and thus

$$
\lim _{n \rightarrow \infty} \widehat{\alpha}_{j}=\Phi^{-1}\left(\Phi\left(\alpha_{j} ; P_{j}(n)\right) ; P_{j}(n)\right)=\alpha_{j}
$$

\section{Appendix B: Additional Results}

Lemma B1 The unit cost function of the Cobb-Douglas production function is

$$
K_{i}\left(S_{i}, A_{i}\left(S_{i}\right), P\right)=\frac{\prod_{j \in S_{i}} P_{j}^{\alpha_{i j}}}{A_{i}\left(S_{i}\right)}
$$


Proof of Lemma B1. Let $X_{i j}^{*}$ and $L_{i}^{*}$ be firm $i$ 's optimal choices of inputs and labor when producing one unit of output. From the firm's first-order conditions, we have $P_{j} X_{i j}^{*}=\alpha_{i j} P_{i}$ and $L_{i}^{*}=\left(1-\sum_{j \in S_{i}} \alpha_{i j}\right) P_{i}$. Dividing the former equation by the latter, we obtain $X_{i j}^{*}=\frac{\alpha_{i j} L_{i}^{*}}{\left(1-\sum_{j \in S_{i}} \alpha_{i j}\right) P_{j}}$. Plugging this into the production function (and recalling that only one unit of output is produced), we obtain

$$
\begin{aligned}
& 1=\frac{1}{\left(1-\sum_{j \in S_{i}} \alpha_{i j}\right)^{1-\sum_{j \in S_{i}} \alpha_{i j}} \prod_{j \in S_{i}} \alpha_{i j}^{\alpha_{i j}}} A_{i}\left(S_{i}\right)\left(L_{i}^{*}\right)^{\left(1-\sum_{j \in S_{i}} \alpha_{i j}\right)} \prod_{j \in S_{i}}\left(\frac{\alpha_{i j} L_{i}^{*}}{\left(1-\sum_{j \in S_{i}} \alpha_{i j}\right) P_{j}}\right)^{\alpha_{i j}} \\
& 1=\frac{1}{\left(1-\sum_{j \in S_{i}} \alpha_{i j}\right)^{1-\sum_{j \in S_{i}} \alpha_{i j}} \prod_{j \in S_{i}} \alpha_{i j}^{\alpha_{i j}}} L_{i}^{*} A_{i}\left(S_{i}\right)\left(1-\sum_{j \in S_{i}} \alpha_{i j}\right)^{-\sum_{j \in S_{i}} \alpha_{i j}} \prod_{j \in S_{i}} \frac{\alpha_{i j}^{\alpha_{i j}}}{P_{j}^{\alpha_{i j}}} \\
& 1=\frac{L_{i}^{*} A_{i}\left(S_{i}\right)}{\left(1-\sum_{j \in S_{i}} \alpha_{i j}\right) \prod_{j \in S_{i}} P_{j}^{\alpha_{i j}}} .
\end{aligned}
$$

Therefore, $L_{i}^{*}=\frac{\left(1-\sum_{j \in S_{i}} \alpha_{i j}\right) \prod_{j \in S_{i}} P_{j}^{\alpha_{i j}}}{A_{i}\left(S_{i}\right)}$. Since $K_{i}\left(S_{i}, A_{i}\left(S_{i}\right), P\right)=P_{i}=\frac{L_{i}^{*}}{\left(1-\sum_{j \in S_{i}} \alpha_{i j}\right)}$, we conclude that $K_{i}\left(S_{i}, A_{i}\left(S_{i}\right), P\right)=\frac{\prod_{j \in S_{i}} P_{j}^{\alpha_{i j}}}{A_{i}\left(S_{i}\right)}$.

Corollary B1 When all industries have Cobb-Douglas production functions and the input-output network is $S$, equilibrium log prices are given as a solution to the following system of linear equations:

$$
p=-(I-\alpha(S))^{-1} a(S)
$$

Proof of Corollary B1. From Lemma B1, $P_{i}=\frac{\prod_{j \in S_{i}} P_{j}^{\alpha_{i j}}}{A_{i}\left(S_{i}\right)}$ for each $i$. Taking logs on both sides, we obtain

$$
p_{i}=\sum_{j \in S_{i}} \alpha_{i j} p_{j}-a_{i}\left(S_{i}\right) \text { for each } i .
$$

From Assumption 1, labor is essential and thus $\sum_{j=1}^{n} \alpha_{i j}<1$ for each $i$. Then, from the Perron-Frobenius Theorem, the matrix $(I-\alpha(S))$ is invertible, and thus $p=-(I-\alpha(S))^{-1} a(S)$.

Lemma B2 (First Borel-Cantelli Lemma) Suppose that $\left\{Z_{n}\right\}_{n \in \mathbb{N}}$ is a sequence of random variables. If for any fixed $\epsilon>0$ we have

$$
\sum_{n=1}^{\infty} \operatorname{Prob}\left[Z_{n}>\epsilon\right]<\infty
$$

then $\lim \sup _{n \rightarrow \infty} Z_{n} \leq 0$ almost surely. 
Lemma B3 (Second Borel-Cantelli Lemma) Suppose that $\left\{Z_{n}\right\}_{n \in \mathbb{N}}$ is a sequence of independent random variables. If

$$
\sum_{n=1}^{\infty} \operatorname{Prob}\left[Z_{n} \geq 0\right]=\infty
$$

then $\lim \sup _{n \rightarrow \infty} Z_{n} \geq 0$ almost surely

Proposition B1 Let $\Phi$ be a Gumbel distribution with $c d f \Phi(z ; \mu, \sigma)=e^{-e^{-\frac{z}{\sigma}}}$. Then $\Phi$ satisfies Assumption 2 with $D=\sigma \log 2$.

Proof. We can write $\lim _{n \rightarrow \infty} \frac{\max _{i \in\{1, \ldots, n\}} Z_{i}}{\log n}$ as $\lim \sup _{n \rightarrow \infty} \frac{Z_{n}}{\log n}$. The probability that $Z_{n}$ is above $\mu+\sigma \log n$ is equal to $1-e^{-e^{-\log n}}=1-e^{-\frac{1}{n}}$. Since $1-e^{-z}=1+z+o(z)$, there exists a constant $\kappa>0$, and an integer $N$ such that for all $n \geq N$ we have $1-e^{-\frac{1}{n}} \geq \frac{\kappa}{n}$. Since $\sum_{n=N}^{\infty} \operatorname{Prob}\left[Z_{n}>\right.$ $\mu+\sigma \log n]>\sum_{n=N}^{\infty} \frac{\kappa}{n}=\infty$ and the variables $Z_{1}, \ldots, Z_{n}$ are independent, we can use Lemma B3 to conclude that $\limsup _{n \rightarrow \infty} \frac{Z_{n}}{\log n} \geq \sigma$ almost surely.

To prove the reverse inequality, let $\epsilon>0$ be arbitrary. The probability that $Z_{n}$ is above $\mu+\sigma(1+\epsilon) \log n$ is $1-e^{-e^{-(1+\epsilon) \log n}}=1-e^{-n^{-1-\epsilon}}$. Since $1-e^{-z}=1+z+o(z)$, there exists a constant $\kappa>0$ and an integer $N$ such that for all $n \geq N$, we have $1-e^{-\frac{1}{n^{1+\epsilon}}} \leq \frac{\kappa}{n^{1+\epsilon}}$. Since $\epsilon>0$ is arbitrary and $\sum_{n=N}^{\infty} \operatorname{Prob}\left[Z_{n} \geq \mu+\sigma(1+\epsilon) \log n\right] \leq \sum_{n=N}^{\infty} \kappa n^{-1-\epsilon}<\infty$, Lemma B2 implies that $\lim \sup _{n \rightarrow \infty} \frac{Z_{n}}{\log n} \leq \sigma$ almost surely. The union of two almost-sure events occurrs almost surely, so we can conclude that $\lim \sup _{n \rightarrow \infty} \frac{Z_{n}}{\log n}=\sigma$ or equivalently $\lim _{n \rightarrow \infty} \frac{\max \left(Z_{1}, \ldots, Z_{n}\right)}{\log n}=\sigma$ almost surely. Using the change of base formula $\log _{2} n=\frac{\log n}{\log 2}$, we obtain $\lim _{n \rightarrow \infty} \frac{\max \left(Z_{1}, \ldots, Z_{n}\right)}{\log _{2} n}=\lim _{n \rightarrow \infty} \frac{\max \left(Z_{1}, \ldots, Z_{n}\right)}{\log n} \log 2=\sigma \log 2$.

Proposition B2 Let $\Phi$ be an exponential distribution with $\operatorname{cdf} \Phi(z ; \lambda)=1-e^{-\lambda z}$. Then $\Phi$ satisfies Assumption 2 with $D=\frac{1}{\lambda} \log 2$.

Proof. We can write $\lim _{n \rightarrow \infty} \frac{\max _{i \in\{1, \ldots, n\}} Z_{i}}{\log n}$ as $\lim \sup _{n \rightarrow \infty} \frac{Z_{n}}{\log n}$. The probability that $Z_{n}$ is above $\frac{\log n}{\lambda}$ is equal to $e^{-\log n}=n^{-1}$. Since $\sum_{n=1}^{\infty} n^{-1}=\infty$ and the variables $Z_{1}, \ldots, Z_{n}$ are independent, Lemma B3 implies that $\lim \sup _{n \rightarrow \infty} \frac{Z_{n}}{\log n} \geq \frac{1}{\lambda}$ almost surely.

To prove the reverse inequality, let $\epsilon>0$ be arbitrary. The probability that $Z_{n}$ is above $\frac{\log n+\epsilon \log n}{\lambda}$ is $e^{-\log n-\epsilon \log n}=n^{-1-\epsilon}$. Since $\epsilon>0$ is arbitrary and $\sum_{n=1}^{\infty} n^{-1-\epsilon}<\infty$, Lemma B2 implies $\lim \sup _{n \rightarrow \infty} \frac{Z_{n}}{\log n} \leq \frac{1}{\lambda}$ almost surely. The intersection of two almost-sure events occurrs almost surely, so we can conclude that $\lim \sup _{n \rightarrow \infty} \frac{Z_{n}}{\log n}=\frac{1}{\lambda}$ or equivalently $\lim _{n \rightarrow \infty} \frac{\max \left(Z_{1}, \ldots, Z_{n}\right)}{\log n}=\frac{1}{\lambda}$ almost surely. Using the change of base formula $\log _{2} n=\frac{\log n}{\log 2}$, we obtain $\lim _{n \rightarrow \infty} \frac{\max \left(Z_{1}, \ldots, Z_{n}\right)}{\log _{2} n}=\lim _{n \rightarrow \infty} \frac{\max \left(Z_{1}, \ldots, Z_{n}\right)}{\log n} \log 2=\frac{1}{\lambda} \log 2$. 


\section{A Model of Hierarchical Input Requirements}

In this part of the Appendix, we generalize our model to incorporate the realistic feature that not all input combinations are feasible for producing each product, and in particular, some industries may have a set of "essential inputs" that are necessary for production. ${ }^{30}$ More specifically, we now extend the model by assuming that there are $K$ categories of inputs, and some (or all) industries will need to use inputs from at least some of these categories. For example, many products will need to use some metals. For simplicity, and to avoid repetition when we show the possibility of sustained growth in this case, we assume Cobb-Douglas production functions throughout this part of the Appendix.

There are $K<\infty$ categories. At each time $t$, one new good in each category arrives, so the total number of goods after $t$ time periods is $t K$. The categories partition the space of goods into $k$ sets $V_{1}(t), \ldots, V_{K}(t)$ so that $\cup_{k=1}^{K} V_{k}(t)=\{1, \ldots, t K\}$. For each industry $i$, there is a set $R_{i} \subset\{1, \ldots, K\}$ of categories that the industry needs to produce. This implies that industry $i$ 's production function is given by family of Cobb-Douglas production functions

$$
Y_{i}=A_{i}\left(S_{i}\right) L_{i}^{1-\sum_{k \in R_{i}} \sum_{j \in S_{i, k}} \alpha_{i j}} \prod_{k \in R_{i}} \prod_{j \in S_{i, k}} X_{i j}^{\alpha_{i j}},
$$

with

$$
S_{i}=\cup_{k} S_{i, k}, S_{i, k} \subset V_{k} \text { and } S_{i, k} \neq \varnothing \text { for each } k \in R_{i}
$$

Our main result is given in the next theorem.

Theorem C1 Suppose that Assumptions 1', 2 and 3 hold, and let D>0 be as defined in Assumption 2. Each industry $i$ chooses sets of suppliers $S_{i, k}^{*}(t) \subset V_{k}(t), S_{i, k}^{*}(t) \neq \varnothing$. Then for each $i \in \mathcal{N}$, the equilibrium log price vector $p^{*}(t)$ satisfies,

$$
\lim _{t \rightarrow \infty}-\frac{p_{i}^{*}(t)}{t K \sum_{j=1}^{t K} \mathcal{L}_{i j}}=D>0 \text { almost surely }
$$

and thus

$$
g^{*}=D K \sum_{i, j=1}^{\infty} \beta_{i} \mathcal{L}_{i j}>0 \text { almost surely, }
$$

Once again, the Leontief inverse, $\mathcal{L}$, that appears in this theorem is different than the one in Theorem 7. The intuition for Theorem C1 can be presented as follows. Even with the restriction that industry $j$ must have one input from each category in $R_{j}$, all possible non-empty combinations of inputs still yields exponentially many draws from the low productivity distribution, and then Assumption 2 ensures sustained (exponential) growth. The proof of this result is similar to that of

\footnotetext{
${ }^{30}$ Assumption 3 imposes that $1-\epsilon$ of the input share of each industry is made up of the first $T$ industries, but this does not imply that any of these first $T$ goods is essential to any industry.
} 
Theorem 7, with the key modification that we do impose that at least one supplier is chosen from each set $V_{k}$, and then prove that the log productivity term $a_{i}\left(S_{i}\right)$ is still almost surely linear in $t$, and the implied Leontief inverse is close to the maximal Leontief inverse. We provide this proof next for completeness.

Proof. Using Lemma A2, for every $\epsilon>0$ there exists $T(\epsilon)$ such that for any $S \supset\{1, \ldots, K T(\epsilon)\}$, $\sum_{j=1}^{t K} \mathcal{L}_{i j}(S) \geq \sum_{j=1}^{t K} \mathcal{L}_{i j}-\frac{1}{(1-\theta)^{2}} \epsilon$.

We now prove that $\liminf _{t \rightarrow \infty}-\frac{p_{i}^{*}(t)}{t K \sum_{j=1}^{t K} \mathcal{L}_{i j}} \geq D$. We will first show that this is the case even if industry $i$ chooses a suboptimal set of inputs corresponding to those with the highest levels of log productivity in each category (rather than the cost-minimizing bundles), and then infer from this that it is also true for the equilibrium price sequence. For each firm $i$, define $S_{i}^{0}(t)=\arg \max _{S_{i} \supset\{1, \ldots, T(\epsilon)\}} A_{i}\left(S_{i}\right)$. Let $S^{0}(t)=\left\{S_{i}^{0}(t)\right\}_{i \in\{1, \ldots, t K\}}$, and define $p_{i}^{0}(t)=$ $-\sum_{j=1}^{t K} \mathcal{L}_{i j}\left(S^{0}(t)\right) a_{i}\left(S_{i}\right)$. The value $a_{i}\left(S_{i}\right)$ is the maximum of $\sum_{r=0}^{\left|R_{i}\right|}(-1)^{r}\left(\begin{array}{c}\left|R_{i}\right| \\ r\end{array}\right) 2^{(t-T(\epsilon))(K-r)}$ independent draws from a distribution $\Phi$ satisfying Assumption $2 .{ }^{31}$ Since $\left|R_{i}\right| \leq K$, and $K$ is a constant independent of $t$, we have that for large $t$ the expression $\sum_{r=0}^{\left|R_{i}\right|}(-1)^{r}\left(\begin{array}{c}\left|R_{i}\right| \\ r\end{array}\right) 2^{(t-T(\epsilon))(K-r)}$ is bounded below by $E 2^{t K}$ where $E$ is a constant independent of $t$. Thus, the value $a_{i}\left(S_{i}\right)$ is the maximum of at least $E 2^{(t-T(\epsilon)) K}$ independent draws from $\Phi$.

Assumption 2 implies that $\lim _{t \rightarrow \infty} \frac{a_{j}\left(S_{j}^{0}(t)\right)}{\log _{2}\left(E 2^{(t-T(\epsilon)) K}\right)} \geq D$ almost surely. Since $T(\epsilon)$ and $E$ are constants independent of $t$, this limit is equal to $\lim _{t \rightarrow \infty} \frac{a_{j}\left(S_{j}^{0}(t)\right)}{t K}$ which is greater than or equal to $D$ almost surely.

Since a countable intersection of almost sure events happens almost surely, we also have $\lim _{t \rightarrow \infty} \min _{j} \frac{a_{j}\left(S_{j}^{0}(t)\right)}{t K} \geq D$ almost surely. Furthermore, since $S_{i}^{0}(t) \supset \varnothing \cup\{1, \ldots, K T(\epsilon)\}$, we have $\sum_{j=1}^{t K} \mathcal{L}_{i j}\left(S^{0}(t)\right) \geq \sum_{j=1}^{t K} \mathcal{L}_{i j}-\frac{1}{(1-\theta)^{2}} \epsilon$. Plugging these bounds into the definition of $p_{i}^{0}$, we obtain

$$
\begin{aligned}
-p_{i}^{0}(t)=\sum_{j=1}^{t K} \mathcal{L}_{i j}(S(t)) a_{j}\left(S_{j}^{0}(t)\right) & \geq \min _{j} a_{j}\left(S_{j}^{0}(t)\right) \sum_{j=1}^{t K} \mathcal{L}_{i j}(S(t)) \\
& \geq \min _{j} a_{j}\left(S_{j}^{0}(t)\right)\left(\sum_{j=1}^{t K} \mathcal{L}_{i j}-\frac{1}{(1-\theta)^{2}} \epsilon\right) .
\end{aligned}
$$

Dividing both sides by $t K \sum_{j=1}^{t K} \mathcal{L}_{i j}$, we obtain

$$
-\frac{p_{i}^{0}(t)}{t K \sum_{j=1}^{t K} \mathcal{L}_{i j}} \geq \frac{\min _{j} a_{j}\left(S_{j}^{0}(t)\right)}{t}-\epsilon \frac{\min _{j} a_{j}\left(S_{j}^{0}(t)\right)}{t K(1-\theta)^{2} \sum_{j=1}^{t K} \mathcal{L}_{i j}} .
$$

\footnotetext{
${ }^{31}$ The expression $\sum_{r=0}^{\left|R_{i}\right|}(-1)^{r}\left(\begin{array}{c}\left|R_{i}\right| \\ r\end{array}\right) 2^{(t-T(\epsilon)) K}-r$ is derived through the exclusion-inclusion principle. To count the sets that firm $i$ can choose, start from all possible $2^{(t-T(\epsilon)) K}$ subsets of inputs that contain $\{1, \ldots, K T(\epsilon)\}$. However, we must remove the $\left(\begin{array}{c}\left|R_{i}\right| \\ 1\end{array}\right) 2^{(t-T(\epsilon))(K-1)}$ sets which do not contain an element in some category $k \in R_{i}$ that the firm needs to produce. If we stopped here, we would be removing sets where two or more categories are absent multiple times, and thus miscounting the number of available sets. Thus, we must add the $\left(\begin{array}{c}\left|R_{i}\right| \\ 2\end{array}\right) 2^{(t-T(\epsilon))(K-2)}$ with two or more sets being absent back into the sum of total feasible sets. Again, this leads to a miscount because sets where three or more categories are absent are being added back to the sum multiple times. Thus, we must remove the $\left(\begin{array}{c}\left|R_{i}\right| \\ 3\end{array}\right) 2^{(t-T(\epsilon))(K-3)}$ sets where 3 or more categories are absent. This procedure keeps going until we reach the set where all elements in all categories in $R_{i}$ are absent.
} 
Using the fact that $\sum_{j=1}^{t} \mathcal{L}_{i j} \geq 1$, this inequality can be written as

$$
-\frac{p_{i}^{0}(t)}{t K \sum_{j=1}^{t K} \mathcal{L}_{i j}} \geq \frac{\min _{j} a_{j}\left(S_{j}^{0}(t)\right)}{t K}-\epsilon \frac{\min _{j} a_{j}\left(S_{j}^{0}(t)\right)}{t K(1-\theta)^{2}} .
$$

Taking liminf on both sides, we obtain

$$
\liminf _{t \rightarrow \infty}-\frac{p_{i}^{0}(t)}{t K \sum_{j=1}^{t K} \mathcal{L}_{i j}} \geq D-\epsilon D \frac{1}{(1-\theta)^{2}} .
$$

Since $\epsilon$ is arbitrarily small, we conclude that

$$
\liminf _{t \rightarrow \infty}-\frac{p_{i}^{0}(t)}{t \sum_{j=1}^{t K} \mathcal{L}_{i j}} \geq D
$$

With the same arguments as in the proof of Theorem 1, we also have that the function $\kappa(p)=$ $\left(\min _{S_{1}} k_{1}\left(a_{1}\left(S_{1}\right), S_{1}, p\right), \ldots, \min _{S_{t K}} k_{t K}\left(a_{t K}\left(S_{t K}\right), S_{t K}, p\right)\right)$ has a smallest fixed point which gives the equilibrium log price vector $p^{*}(t)$. Starting from $p^{0}(t)$, we can define a decreasing sequence $p^{\tau}(t)=$ $\kappa\left(p^{\tau-1}(t)\right)$ which converges to a fixed point $p(t)$ of $\kappa$. Since the equilibrium log price vector is the lowest fixed point of $\kappa$, we have that $p^{*}(t) \leq p(t) \leq p^{0}(t)$. Dividing by $t K \sum_{j=1}^{t K} \mathcal{L}_{i j}$ and taking liminf on both sides, we conclude

$$
\liminf _{t \rightarrow \infty}-\frac{p_{i}^{*}(t)}{t K \sum_{j=1}^{t K} \mathcal{L}_{i j}} \geq D
$$

To prove that $\lim \sup _{t \rightarrow \infty} \frac{-p_{i}^{*}(t)}{t K \sum_{j=1}^{t K} \mathcal{L}_{i j}} \leq D$, let us write $-p_{i}^{*}(t)=\sum_{j=1}^{t K} \mathcal{L}_{i j}(S(t)) a_{j}\left(S_{j}(t)\right) \leq$ $\max _{j} a_{j}\left(S_{j}(t)\right) \sum_{j=1}^{t K} \mathcal{L}_{i j}$. The value of $\max _{j} a_{j}\left(S_{j}(t)\right)$ can be upper bounded by $\max _{j} \max _{S_{j}^{\prime}} a_{j}\left(S_{j}^{\prime}\right)$, which is the maximum of $t K \times 2^{t K}$ i.i.d. draws from the distribution $\Phi$. From Assumption 2, we have $\limsup \sup _{t \rightarrow \infty} \frac{\max _{j} \max _{S_{j}^{\prime}} a_{j}\left(S_{j}^{\prime}\right)}{\log _{2}(t K)+t K} \leq D$. Since $\log _{2}(t K)=o(t K)$, we have that $\limsup \sup _{t \rightarrow \infty} \frac{\max _{j} \max _{S_{j}^{\prime}} a_{j}\left(S_{j}^{\prime}\right)}{t K} \leq D$. Dividing $-p_{i}^{*}(t)$ by $t K \sum_{j=1}^{t K} \mathcal{L}_{i j}$ and taking limsup on both sides, we obtain

$$
\limsup _{t \rightarrow \infty}-\frac{p_{i}(t)}{t K \sum_{j=1}^{t K} \mathcal{L}_{i j}} \leq \limsup _{t \rightarrow \infty} \frac{\max _{j} \max _{S_{j}^{\prime}} a_{j}\left(S_{j}^{\prime}\right)}{t K} \leq D \text { almost surely. }
$$

Combining this with $(\mathrm{C} 1)$, we can thus conclude that

$$
\lim _{t \rightarrow \infty}-\frac{p_{i}^{*}(t)}{t K \sum_{j=1}^{t K} \mathcal{L}_{i j}}=D \text { almost surely }
$$

and thus

$$
g^{*}=\lim _{t \rightarrow \infty}\left(-\frac{\pi(t)}{t}\right)=D K \sum_{i, j=1}^{\infty} \beta_{i} \mathcal{L}_{i j} \text { almost surely }
$$




\section{Appendix D: Imputing Removal Probabilities from OECD Data}

In our counterfactual simulations in Section 7, we use OECD data to impute sectoral marginal distortions. The OECD provides input-output tables for 63 countries at the level of 34 sectors as well as the tax share of intermediate input spending in each industry and each country. ${ }^{32}$ The average tax share across all developing countries and all sectors is $1.4 \%$, which we impute as the average marginal distortion in our counterfactual simulation. We also simulate our model with a very high level of $\tau=10 \%$, which is approximately four times as high as the average tax in the OECD data, and approximately twice as large as the highest observed tax (Saudi Arabia has an average intermediate input tax share of $6.11 \%$ ).

We also use the OECD data to estimate average probabilities with which input-output linkages present in the US data are missing in the production networks of developing countries. Since the effects of reasonable marginal distortions appear to be small on the production network, we take these probabilities as a representation of the direct restrictions on input-output linkages. To estimate these probabilities, we first verify that the OECD input-output table for the United States matches the data provided in the BEA input-output table with 377 industries. Following Acemoglu, Ozdaglar and Tahbaz-Salehi (2017), we use uppercase letters to refer to sectors in the aggregate OECD data, and lowercase letters to refer to industries in the disaggregated BEA data. We use a crosswalk to match each industry $i$ in the BEA data to a unique sector $I$ in the OECD data. We denote this relation by $i \in I$. For any sectors $I, J$ in the aggregate data, the following flow conditions must hold

$$
\begin{gathered}
P_{J} X_{I J}=\sum_{i \in I} \sum_{j \in J} P_{j} X_{i j} \\
P_{I} C_{I}=\sum_{i \in I} P_{i} C_{i} \\
w L_{I}=\sum_{i \in I} w L_{i}
\end{gathered}
$$

Let $v_{i}=\frac{P_{i} Y_{i}}{G D P}$ and let $v_{I}=\frac{P_{I} Y_{I}}{G D P}$ be the industry and sectoral Domar weights. Acemoglu, Ozdaglar and Tahbaz-Salehi (2017) establish:

Lemma D1 For any two sectors $I, J$ in the aggregate data, we have

$$
v_{I} \alpha_{I J}=\sum_{i \in I} \sum_{j \in J} \alpha_{i j} v_{i}
$$

Let $\widetilde{\alpha}_{I J}=\sum_{i \in I} \sum_{j \in J} \alpha_{i j} \frac{v_{i}}{v_{I}}$. We can compute this expression using the BEA data, and compare it to the value of $\alpha_{I J}$ observed in the OECD data. In particular, we compare the vectors of indegrees

\footnotetext{
${ }^{32}$ The intermediate input tax share is obtained by dividing the row "Taxes less subsidies on intermediate and final products" by the row "Total intermediate and final expenditure at purchasers' prices".
} 
$\left\{\sum_{J} \alpha_{I J}\right\}_{I},\left\{\sum_{J} \widetilde{\alpha}_{I J}\right\}_{I}$ and the vectors of outdegrees $\left\{\sum_{I} \alpha_{I J}\right\}_{J},\left\{\sum_{I} \widetilde{\alpha}_{I J}\right\}_{J}$. Using a KolmogorovSmirnov test, we obtain that the probability that the vectors of indegrees in the BEA and OECD data are drawn from the same distribution is 0.999 . The probability that the vectors of outdegrees are drawn from the same distribution is 0.962. Furthermore, the average values of $\frac{1}{N^{2}} \sum_{I, J} \alpha_{I J}$ and $\frac{1}{N^{2}} \sum_{I, J} \widetilde{\alpha}_{i j}$ are both equal to 0.015 . The root mean squared error $\sqrt{\frac{1}{N^{2}} \sum_{I, J}\left(\alpha_{I J}-\widetilde{\alpha}_{I J}\right)^{2}}$ is equal to 0.014 .

This evidence thus suggests that the OECD aggregate data for the United States matches the disaggregated BEA data for the United States. Motivated by this, we then treat the OECD data for other countries as an aggregate of an unobserved disaggregate input-output matrix. For each country $c$, we impute a probability that an edge $(i, j)$ observed in the US data would be missing in that country's disaggregated input-output matrix. Let us assume that the disaggregate Domarweighted exogneous input-output shares $v_{i} \alpha_{i j}$ are the same across countries, but each edge $(i, j)$ present in the US input-output network is missing from the data in country $c$ with probability $\rho_{c}$. Then we have

$$
\frac{v_{I, c} \alpha_{I J, c}}{v_{I, U S} \alpha_{I J, U S}}=\frac{\sum_{i \in I, j \in J}\left(1-\rho_{c}\right) \alpha_{i j} v_{i, c}}{\sum_{i \in I, j \in J} \alpha_{i j} v_{i, U S}}=1-\rho_{c} .
$$

This parameter $\rho_{c}$ can be estimated as $\widehat{\rho}_{c}=1-\frac{1}{N} \sum_{J=1}^{N}\left(\frac{\sum_{I=1}^{N} v_{I, c} \alpha_{I J, c}}{\sum_{I=1}^{N} v_{I, U S} \alpha_{I J, U S}}\right)$. For the 22 countries catalogued in the OECD data as developing countries, we obtain that $1-\widehat{\rho}_{c}$ ranges from 0.321 to 1.537 and has an average value of 0.891 . 


\section{References}

[1] Acemoglu, D., Autor, D. and Patterson C. (2017). Technological bottlenecks and the productivity puzzle. Manuscript.

[2] Acemoglu, D., Carvalho, V. M., Ozdaglar, A., and Tahbaz-Salehi, A. (2012). The network origins of aggregate fluctuations. Econometrica, 80(5), 1977-2016

[3] Acemoglu, D., Ozdaglar, A., and Tahbaz-Salehi, A. (2017). Microeconomic origins of macroeconomic tail risks. The American Economic Review, 107(1), 54-108.

[4] Aghion, P., and Howitt, P. (1992). A model of Growth through Creative Destruction.Econometrica, 60(2), 323-351.

[5] Auerswald, P., Kauffman, S., Lobo, J., \& Shell, K. (2000). The production recipes approach to modeling technological innovation: An application to learning by doing. Journal of Economic Dynamics and Control, 24(3), 389-450.

[6] Baqaee, D. R. (2016). Cascading failures in production networks. Manuscript.

[7] Baqaee, D. R., and Farhi, E. (2017). The Macroeconomic Impact of Microeconomic Shocks: Beyond Hulten's Theorem . National Bureau of Economic Research Working Paper No. w23145.

[8] Bigio, S., and La'O, J. (2016). Financial frictions in production networks. National Bureau of Economic Research Working Paper No. 22212.

[9] Caliendo, L., Parro, F., and Tsyvinski, A. (2017). Distortions and the Structure of the World Economy. National Bureau of Economic Research Working Paper No. w23332.

[10] Bartelme, D., and Gorodnichenko Y. (2015) Linkages and Economic Development. National Bureau of Economic Research Working Paper No. 21251.

[11] Eaton, J., and Kortum, S. (2001). Technology, trade, and growth: A unified framework. European economic review, 45(4), 742-755.

[12] Fadinger, H., Ghiglino C. and Teteryatnikova M. (2016) Income differences and input-output structure. CEPR Discussion Paper No. $1154 \%$.

[13] Gabaix, X. (2011). The granular origins of aggregate fluctuations. Econometrica, 79(3), 733772 .

[14] Ghiglino, C. (2012). Random walk to innovation: Why productivity follows a power law. Journal of Economic Theory, 147(2), 713-737. 
[15] Grossman, G. M., and Helpman, E. (1991). Quality ladders in the theory of growth. The Review of Economic Studies, 58(1), 43-61.

[16] Grossman, G. M., and Helpman, E. (1992). Innovation and growth in the global economy. MIT press.

[17] IMF (2015). World Economic Outlook.

[18] Jones, C. I. (1995). R \& D-based models of economic growth. Journal of political Economy, 103(4), 759-784.

[19] Jones, C. I. (2011). Intermediate goods and weak links in the theory of economic development. American Economic Journal: Macroeconomics, 3(2), 1-28.

[20] Kennan, J. (2001). Uniqueness of Positive Fixed Points for Increasing Concave Functions on $\mathbb{R}^{n}$ : An Elementary Result. Review of Economic Dynamics, 4(4), 893-899.

[21] Klette, T. J., and Kortum, S. (2004). Innovating firms and aggregate innovation. Journal of political economy, 112(5), 986-1018.

[22] Liu, E. (2017). Industrial policies and economic development. Manuscript.

[23] Long, J. B., and Plosser, C. I. (1983). Real business cycles. Journal of political Economy, 91(1), 39-69. Chicago

[24] Lucas, R. E. (2009). Ideas and growth. Economica, 76(301), 1-19.

[25] Lucas, R. E., and Moll, B. (2014). Knowledge growth and the allocation of time. Journal of Political Economy, 122(1), 1-51.

[26] McFadden, D. (1973). Conditional logit analysis of qualitative choice behavior. Frontiers of Econometrics, ed. by P. Zambreka. New York: Academic Press.

[27] Oberfield, E. (2017). A theory of input-output architecture. Technical report.

[28] Perla, J., and Tonetti, C. (2014). Equilibrium imitation and growth. Journal of Political Economy, 122(1), 52-76.

[29] Romer, P. M. (1990). Endogenous technological change. Journal of political Economy, 98(5, Part 2), S71-S102.

[30] Samuelson, P. A. (1951). Abstract of a theorem concerning substitutability in open Leontief models. Activity analysis of production and allocation, 13, 142-6. 
[31] Taschereau-Dumouchel, M. (2017). Cascades and fluctuations in an economy with an endogenous production network.Manuscript

[32] Topkis, D. M. (1998). Supermodularity and Complementarity. Princeton University Press.

[33] Weitzman, M. L. (1998). Recombinant growth. The Quarterly Journal of Economics, 113(2), 331-360. 